\title{
Efficient Syntheses of New Polyhydroxylated 2,3-Diaryl-9H-xanthen-9-ones
}

\author{
Clementina M. M. Santos, ${ }^{[a, b]}$ Artur M. S. Silva, ${ }^{*[b]}$ and José A. S. Cavaleiro ${ }^{[b]}$
}

Keywords: Xanthones / Alcohols / Oxygen heterocycles / Heck reaction / NMR spectroscopy

\begin{abstract}
A large number of hydroxylated 2,3-diaryl-9H-xanthen-9ones have been synthesised by two different approaches, starting either from 3-bromo-2-methyl-4H-chromen-4-one or from (E)-3-bromo-2-styryl-4H-chromen-4-ones. The former method involves Heck reactions between 3-bromo-2-methyl$4 H$-chromen-4-one and styrenes, leading to (E)-2-methyl-3styryl-4H-chromen-4-ones; these condensed with benzaldehyde to give $(E, E)$-2,3-distyryl-4H-chromen-4-ones, which led to the desired 2,3-diaryl-9H-xanthen-9-ones under reflux in 1,2,4-trichlorobenzene. 3-Bromo-2-styryl-4H-chromen-4ones were obtained either by aldol condensations between 3-bromo-2-methyl-4H-chromen-4-one and benzaldehydes, or through Baker-Venkataraman rearrangements of 2-acetylphenyl cinnamates, followed by one-pot bromination/ cyclisation with phenyltrimethylammonium tribromide. The 2,3-diaryl-9H-xanthene-9-ones were obtained in one-pot transformations involving Heck reactions between $(E)$-3-
\end{abstract}

\section{Introduction}

Xanthones constitute one of the major classes of naturally occurring oxygen-containing heterocyclic compounds containing dibenzo- $\gamma$-pyrone rings. ${ }^{[1]}$ They occur in two major plant families, Guttiferae and Gentianaceae, and also in some families of fungi and lichens..$^{[2,3]}$ Natural derivatives can be hydroxylated, methoxylated or prenylated, among other possibilities; the parent compound xanthone itself is not known as a natural product. ${ }^{[3,4]}$ The presence of aryl groups on the xanthone core has only been reported for a few synthetic derivatives, and as far as we know the literature had never presented the synthesis of xanthones featuring 2,3-diaryl moieties before our work. ${ }^{[5,6]}$

Over the last decades these substances have been extensively studied not only because they participate in several biological functions but also as a consequence of their remarkable antifungal, ${ }^{[7-9]}$ anti-inflammatory, ${ }^{[10,11]}$ antimalaria ${ }^{[12-14]}$ and antitumour activities, ${ }^{[15,16]}$ and even as promising antioxidant agents. ${ }^{[17-19]}$ Structure-activity stud-

[a] Department of Vegetal Production and Technology, Escola Superior Agrária de Bragança,

5301-855 Bragança, Portugal

Fax: +351-273-325405

E-mail: clems@ipb.pt

[b] Department of Chemistry \& QOPNA, University of Aveiro, 3810-193 Aveiro, Portugal

Fax: +351-234-370084

E-mail: artur.silva@ua.pt
bromo-2-styryl-4H-chromen-4-ones and styrenes, followed by electrocyclisation and oxidation processes. The 2,3-diaryl3,4-dihydro-9H-xanthene-9-one intermediates were also isolated under these conditions, and so when 5-methoxy-2-styryl-4H-chromen-4-ones were used as starting materials the 1-hydroxy-6,7-diaryl-9H-xanthene-9-ones were also observed. The second method is a general one, because it allowed the synthesis of a great number of 2,3-diaryl-9Hxanthen-9-ones with several substitution patterns, whereas the first one is limited to certain derivatives. The last step in the synthesis of hydroxylated 2,3-diaryl-9H-xanthen-9-ones was the cleavage of the hydroxy protecting groups with boron tribromide. The structures and stereochemistry of all new compounds were established by NMR studies.

ies of phenolic compounds have revealed that features conducive to high antioxidant activity are the presence of hydroxy groups at certain positions on the skeleton, a catechol group being the most prominent moiety. ${ }^{[20,21]}$ In view of these considerations, here we report two main routes for the synthesis of polyhydroxylated 2,3-diaryl-9H-xanthen-9ones.

There are various known methods for the synthesis of $9 H$-xanthen-9-ones. ${ }^{[22]}$ The classic ones involve the bonding of two benzene rings through a pyran unit. The most commonly reported are Friedel-Crafts acylations of phenolic derivatives with benzoyl chlorides and Ullmann condensation between sodium phenolates and ortho-halo-substituted benzoic acids for the synthesis of diaryl ethers, with both methods being followed by cyclisations of these compounds to form the heterocyclic rings. The literature also reports direct formation of the $9 H$-xanthen-9-one nucleus through a diaryl ester pyrolysis process or by photo-Fries or Smiles rearrangements of these intermediates, accompanied by ring closure. These classical methods require some drastic conditions, give poor yields because of the formation of several by-products and only allow the presence of certain substituents in certain positions on the $9 H$-xanthen-9-one core. $^{[22-24]}$

Modifications of the classical methods and several other less traditional approaches to construction of the $9 \mathrm{H}$ xanthen-9-one framework have been widely described, but 


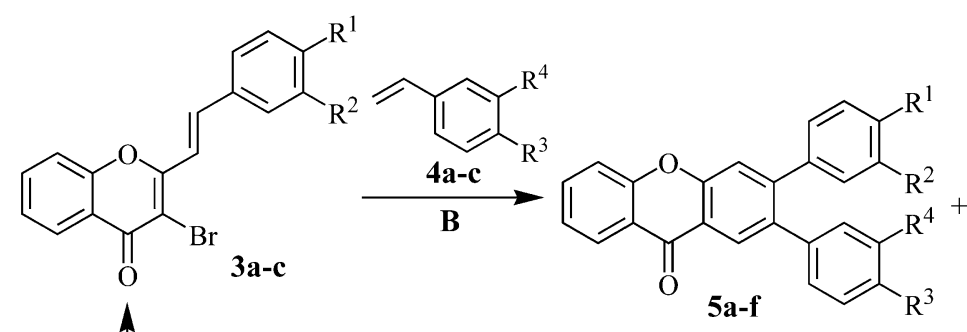<smiles>[R]c1ccc(C2=Cc3c(oc4cc[c-]cc4c3=O)C[C@H]2c2ccc([R])c([R2])c2)cc1[Y]</smiles><smiles>[Y]c1ccc(C=CC)cc1[14CH3]</smiles><smiles>[R]c1cc(C=C)cc([14CH]=C)c1</smiles><smiles>O=c1c2c(oc3ccccc13)C=C(c1ccccc1)[C@H](c1ccccc1)[C@H]2c1ccccc1</smiles>

A,D: $\mathrm{MeOH}, \mathrm{Na}$, room temp., $48 \mathrm{~h}$

B: $\mathrm{NMP}, \mathrm{Pd}\left(\mathrm{PPh}_{3}\right)_{4}, \mathrm{PPh}_{3}, \mathrm{Et}_{3} \mathrm{~N}, 160^{\circ} \mathrm{C}$ to reflux

C: $\mathrm{NMP}, \mathrm{PdCl}_{2}, \mathrm{PPh}_{3}, \mathrm{Et}_{3} \mathrm{~N}, 160^{\circ} \mathrm{C}, 9 \mathrm{~h}$

E: 1,2,4-Trichlorobenzene, reflux, $18 \mathrm{~h}$

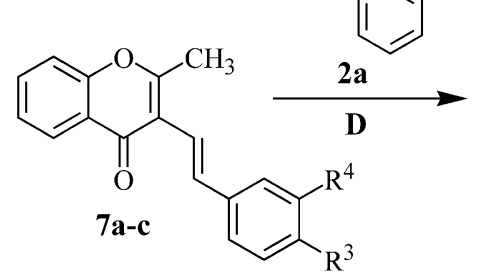

2a-c, 3a-c
a) $\mathrm{R}^{1}=\mathrm{R}^{2}=\mathrm{H}$
b) $\mathrm{R}^{1}=\mathrm{OBn}, \mathrm{R}^{2}=\mathrm{H}$
5a-f, 6a-f, 8a-c
c) $\mathrm{R}^{1}=\mathrm{R}^{2}=\mathrm{OBn}$
a) $\mathrm{R}^{1}=\mathrm{R}^{2}=\mathrm{R}^{3}=\mathrm{R}^{4}=\mathrm{H}$
$4 a-c, 7 a-c$
a) $\mathrm{R}^{1}=\mathrm{R}^{2}=\mathrm{H}$
b) $\mathrm{R}^{1}=\mathrm{R}^{2}=\mathrm{H}, \mathrm{R}^{3}=\mathrm{OMe}, \mathrm{R}^{4}=\mathrm{H}$
d) $\mathrm{R}^{1}=\mathrm{OBn}, \mathrm{R}^{2}=\mathrm{R}^{3}=\mathrm{R}^{4}=\mathrm{H}$
b) $\mathrm{R}^{1}=\mathrm{OMe}, \mathrm{R}^{2}=\mathrm{H}$ e) $\mathrm{R}^{1}=\mathrm{OBn}, \mathrm{R}^{2}=\mathrm{H}, \mathrm{R}^{3}=\mathrm{OMe}, \mathrm{R}^{4}=\mathrm{H}$
c) $\mathrm{R}^{1}=\mathrm{R}^{2}=\mathrm{OMe}$
f) $\mathrm{R}^{1}=\mathrm{OBn}, \mathrm{R}^{2}=\mathrm{H}, \mathrm{R}^{3}=\mathrm{R}^{4}=\mathrm{OMe}$

Scheme 1. Synthesis of 2,3-diaryl-9H-xanthen-9-ones 5a-f.

none of them allows the synthesis of our target compounds, the 2,3-diaryl-9H-xanthen-9-ones. ${ }^{[22]}$ In this context, we have developed two methods for the synthesis of new polyhydroxylated 2,3-diaryl-9H-xanthen-9-ones, starting from 3-bromo-2-methyl-4H-chromen-4-one (1, Scheme 1) and the (E)-3-bromo-2-styryl-4H-chromen-4-ones 3a-k (Scheme 2).

\section{Results and Discussion}

\section{Syntheses}

Our first approach to the synthesis of 2,3-diaryl- $9 H$ xanthen-9-ones involved the use of the functionalised 3bromo-2-methyl- $4 H$-chromen-4-one (1), which was prepared from 2'-hydroxyacetophenone in a three-step sequence and in a good overall yield. ${ }^{[25-26]}$ Two methods to obtain the 2,3-diaryl-9H-xanthen-9-ones 5a-f were developed (Scheme 1). In one of these, the (E)-3-bromo-2-styryl$4 H$-chromen-4-ones $\mathbf{3 a - c}$ were prepared through base-catalysed aldol reactions between $4 H$-chromen-4-one 1 and benzaldehydes $\mathbf{2 a - c}$ in methanolic solutions. The best results were achieved when 4 molar equivalents of base and reaction times of $48 \mathrm{~h}$ were used. Even after several attempts, the yields of $(E)-3^{\prime}, 4^{\prime}$-dibenzyloxy-3-bromo-2-styryl-4H-chromen-4-one (3c) never exceeded $6 \%$ and its isolation and characterisation was not possible, due to its degradation in chloroform solution. ${ }^{[25]}$ The next step consisted of Heck reactions between the $(E)$-3-bromo-2-styryl- $4 \mathrm{H}$ - chromen-4-ones $\mathbf{3 a}$ and $\mathbf{3 b}$ and styrenes $\mathbf{4} \mathbf{a}-\mathbf{c}$ in the presence of tetrakis(triphenylphosphane)palladium(0) as catalyst, directly giving the $9 H$-xanthen-9-ones 5a-f (see Exp. Section, Table 2). Under these conditions it was also possible in each case to isolate a minor compound with lower $R_{\mathrm{f}}$ value, corresponding to the 2,3-diaryl-3,4-dihydro-9H-xanthen-9-one intermediates $\mathbf{6 a}-\mathbf{f}$.

An alternative method for the synthesis of 2,3-diaryl-9Hxanthen-9-ones started with Heck reactions between 3bromo-2-methyl-4H-chromen-4-one (1) and styrenes $4 \mathbf{4 a - c}$ (Scheme 1). Several attempts to optimise the coupling reaction conditions were made, and the best results for the synthesis of the (E)-2-methyl-3-styryl-4H-chromen-4-ones 7ac were achieved with 1 molar equivalent of base, 0.1 molar equivalent of phosphane and 5 molar equivalents of styrenes $4 \mathrm{a}-\mathbf{c}$ over $9 \mathrm{~h}$ at $160{ }^{\circ} \mathrm{C}$. Unlike in the coupling reaction described above, in which the best results were achieved with tetrakis(triphenylphosphane)palladium $(0)$, here the best yields were obtained in the presence of palladium chloride as catalyst (Table 1 ).

The (E,E)-2,3-distyryl-4H-chromen-4-ones 8a-c were prepared through base-catalysed aldol condensations between the $(E)$-2-methyl-3-styryl-4H-chromen-4-ones 7a-c and benzaldehyde (2a) in the presence of 4 molar equivalents of base over $48 \mathrm{~h}$ at room temperature. Condensations between the (E)-2-methyl-3-styryl-4H-chromen-4-ones 7a-c and the benzaldehydes $\mathbf{2 b}$ and $\mathbf{2 c}$ were unsuccessful, probably due to poor reactivity of benzyloxybenzaldehydes $\mathbf{2 b}$ 
Table 1. Yields for the formation of (E)-2-methyl-3-styryl-4Hchromen-4-ones $\mathbf{7 a - c}$ through Heck reactions between 3-bromo-2methyl-4H-chromen-4-one (1) and styrenes $\mathbf{4 a - c .}$

\begin{tabular}{lccc}
\hline Catalyst [equiv.] & \% Yield 7a & \% Yield 7b & $\%$ Yield 7c \\
\hline $\mathrm{Pd}(\mathrm{OAc})_{2}(0.05)$ & 46 & 28 & 17 \\
$\mathrm{Pd}\left(\mathrm{PPh}_{3}\right)_{4}(0.05)$ & 48 & 43 & 30 \\
$\mathrm{PdCl}_{2}(0.05)$ & 48 & 49 & 52 \\
\hline
\end{tabular}

and 2c. The 2,3-diaryl-9H-xanthen-9-ones 5a-c were then obtained by heating the $(E, E)$-2,3-distyryl-4H-chromen-4ones $\mathbf{8 a}-\mathbf{c}$ in 1,2,4-trichlorobenzene at reflux. In the case of the unsubstituted 2,3-distyryl-4H-chromen-4-one 8a, TLC analysis of the reaction medium after a reaction time of $12 \mathrm{~h}$ revealed the presence of three compounds, with the major one with the highest $R_{\mathrm{f}}$ value corresponding to the desired 2,3-diphenyl-9H-xanthen-9-one 5a, together with two other minor compounds. Their NMR spectra showed that the minor product with high $R_{\mathrm{f}}$ was 2,3-diphenyl-3,4dihydro-9H-xanthen-9-one (6a) and the other, with a lower $R_{\mathrm{f}}$, was 2,3-diphenyl-1,2-dihydro-9H-xanthen-9-one (9a). After $18 \mathrm{~h}$, however, only the desired 2,3-diphenyl-9Hxanthen-9-one (5a) was obtained.

In view of the lack of reactivity in some steps in the two previously described methods, we developed a third and more general method for the synthesis of 2,3-diaryl-9Hxanthen-9-ones, starting from the 2-acetylphenyl cinnamates $\mathbf{1 0 a}-\mathbf{i}$ (Scheme 2). ${ }^{[27]}$ The cinnamates $\mathbf{1 0 a}-\mathbf{i}$ were obtained in good yields from reactions between acetophenones and cinnamic acid derivatives. Baker-Venkataraman<smiles>[R]c1cc([R])c(OC(=O)/C=C/c2ccc([R])c([R])c2)c(C(C)=O)c1</smiles>

A: DMSO, KOH, room temp., $2 \mathrm{~h}$

B: THF, PTT, room temp., $12 \mathrm{~h}$

C: $\mathrm{NMP}, \mathrm{Pd}\left(\mathrm{PPh}_{3}\right)_{4}, \mathrm{PPh}_{3}, \mathrm{Et}_{3} \mathrm{~N}, 160^{\circ} \mathrm{C}$ to reflux

\section{0a-i, 11a-i}

a) $\mathrm{R}^{1}=\mathrm{R}^{2}=\mathrm{R}^{5}=\mathrm{R}^{6}=\mathrm{H}$

b) $\mathrm{R}^{1}=\mathrm{OMe}, \mathrm{R}^{2}=\mathrm{R}^{5}=\mathrm{R}^{6}=\mathrm{H}$

c) $\mathrm{R}^{1}=\mathrm{R}^{2}=\mathrm{OMe}, \mathrm{R}^{5}=\mathrm{R}^{6}=\mathrm{H}$

d) $\mathrm{R}^{1}=\mathrm{R}^{2}=\mathrm{H}, \mathrm{R}^{5}=\mathrm{OMe}, \mathrm{R}^{6}=\mathrm{H}$

e) $\mathrm{R}^{1}=\mathrm{R}^{2}=\mathrm{R}^{5}=\mathrm{OMe}, \mathrm{R}^{6}=\mathrm{H}$

f) $\mathrm{R}^{1}=\mathrm{R}^{2}=\mathrm{R}^{5}=\mathrm{H}, \mathrm{R}^{6}=\mathrm{OMe}$

g) $\mathrm{R}^{1}=\mathrm{R}^{2}=\mathrm{OMe}, \mathrm{R}^{5}=\mathrm{H}, \mathrm{R}^{6}=\mathrm{OMe}$

h) $\mathrm{R}^{1}=\mathrm{R}^{2}=\mathrm{H}, \mathrm{R}^{5}=\mathrm{R}^{6}=\mathrm{OMe}$

i) $\mathrm{R}^{1}=\mathrm{R}^{2}=\mathrm{R}^{5}=\mathrm{R}^{6}=\mathrm{OMe}$

\section{3a,d-k}

a) $\mathrm{R}^{1}=\mathrm{R}^{2}=\mathrm{R}^{5}=\mathrm{R}^{6}=\mathrm{H}$

d) $\mathrm{R}^{1}=\mathrm{OMe}, \mathrm{R}^{2}=\mathrm{R}^{5}=\mathrm{R}^{6}=\mathrm{H}$

e) $\mathrm{R}^{1}=\mathrm{R}^{2}=$ OMe, $\mathrm{R}^{5}=\mathrm{R}^{6}=\mathrm{H}$

f) $\mathrm{R}^{1}=\mathrm{R}^{2}=\mathrm{H}, \mathrm{R}^{5}=\mathrm{OMe}, \mathrm{R}^{6}=\mathrm{H}$

g) $\mathrm{R}^{1}=\mathrm{R}^{2}=\mathrm{R}^{5}=\mathrm{OMe}, \mathrm{R}^{6}=\mathrm{H}$

h) $\mathrm{R}^{1}=\mathrm{R}^{2}=\mathrm{R}^{5}=\mathrm{H}, \mathrm{R}^{6}=\mathrm{OMe}$

i) $\mathrm{R}^{1}=\mathrm{R}^{2}=\mathrm{OMe}, \mathrm{R}^{5}=\mathrm{H}, \mathrm{R}^{6}=\mathrm{OMe}$

j) $\mathrm{R}^{1}=\mathrm{R}^{2}=\mathrm{H}, \mathrm{R}^{5}=\mathrm{R}^{6}=\mathrm{OMe}$

k) $\mathrm{R}^{1}=\mathrm{R}^{2}=\mathrm{R}^{5}=\mathrm{R}^{6}=\mathrm{OMe}$ 5a-c,g-s, 6a-c,g-s

a) $\mathrm{R}^{1}=\mathrm{R}^{2}=\mathrm{R}^{3}=\mathrm{R}^{4}=\mathrm{R}^{5}=\mathrm{R}^{6}=\mathrm{H}$

b) $\mathrm{R}^{1}=\mathrm{R}^{2}=\mathrm{H}, \mathrm{R}^{3}=$ OMe, $\mathrm{R}^{4}=\mathrm{R}^{5}=\mathrm{R}^{6}=\mathrm{H}$

c) $\mathrm{R}^{1}=\mathrm{R}^{2}=\mathrm{H}, \mathrm{R}^{3}=\mathrm{R}^{4}=\mathrm{OMe}, \mathrm{R}^{5}=\mathrm{R}^{6}=\mathrm{H}$

g) $\mathrm{R}^{1}=\mathrm{OMe}, \mathrm{R}^{2}=\mathrm{R}^{3}=\mathrm{R}^{4}=\mathrm{R}^{5}=\mathrm{R}^{6}=\mathrm{H}$

h) $\mathrm{R}^{1}=$ OMe, $\mathrm{R}^{2}=\mathrm{H}, \mathrm{R}^{3}=$ OMe, $\mathrm{R}^{4}=\mathrm{R}^{5}=\mathrm{R}^{6}=\mathrm{H}$

i) $\mathrm{R}^{1}=\mathrm{OMe}, \mathrm{R}^{2}=\mathrm{H}, \mathrm{R}^{3}=\mathrm{R}^{4}=\mathrm{OMe}, \mathrm{R}^{5}=\mathrm{R}^{6}=\mathrm{H}$

j) $\mathrm{R}^{1}=\mathrm{R}^{2}=\mathrm{OMe}, \mathrm{R}^{3}=\mathrm{R}^{4}=\mathrm{R}^{5}=\mathrm{R}^{6}=\mathrm{H}$

k) $\mathrm{R}^{1}=\mathrm{R}^{2}=\mathrm{R}^{3}=$ OMe, $\mathrm{R}^{4}=\mathrm{R}^{5}=\mathrm{R}^{6}=\mathrm{H}$

l) $\mathrm{R}^{1}=\mathrm{R}^{2}=\mathrm{R}^{3}=\mathrm{R}^{4}=$ OMe, $\mathrm{R}^{5}=\mathrm{R}^{6}=\mathrm{H}$

m) $R^{1}=R^{2}=R^{3}=R^{4}=H, R^{5}=O M e, R^{6}=H$

n) $\mathrm{R}^{1}=\mathrm{R}^{2}=\mathrm{R}^{3}=\mathrm{R}^{4}=\mathrm{R}^{5}=\mathrm{H}, \mathrm{R}^{6}=\mathrm{OMe}$

o) $\mathrm{R}^{1}=\mathrm{R}^{2}=\mathrm{H}, \mathrm{R}^{3}=\mathrm{OMe}, \mathrm{R}^{4}=\mathrm{R}^{5}=\mathrm{H}, \mathrm{R}^{6}=\mathrm{OMe}$

p) $\mathrm{R}^{1}=\mathrm{R}^{2}=\mathrm{H}, \mathrm{R}^{3}=\mathrm{R}^{4}=\mathrm{OMe}, \mathrm{R}^{5}=\mathrm{H}, \mathrm{R}^{6}=\mathrm{OMe}$

q) $\mathrm{R}^{1}=\mathrm{R}^{2}=\mathrm{OMe}, \mathrm{R}^{3}=\mathrm{R}^{4}=\mathrm{R}^{5}=\mathrm{H}, \mathrm{R}^{6}=\mathrm{OMe}$

r) $\mathrm{R}^{1}=\mathrm{R}^{2}=\mathrm{R}^{3}=\mathrm{R}^{4}=$ OMe, $\mathrm{R}^{5}=\mathrm{H}, \mathrm{R}^{6}=\mathrm{OMe}$

s) $\mathrm{R}^{1}=\mathrm{R}^{2}=\mathrm{R}^{3}=\mathrm{R}^{4}=\mathrm{H}, \mathrm{R}^{5}=\mathrm{R}^{6}=\mathrm{OMe}$

12n-s

n) $\mathrm{R}^{1}=\mathrm{R}^{2}=\mathrm{R}^{3}=\mathrm{R}^{4}=\mathrm{R}^{5}=\mathrm{H}$

o) $R^{1}=R^{2}=H, R^{3}=$ OMe, $R^{4}=R^{5}=H$

p) $\mathrm{R}^{1}=\mathrm{R}^{2}=\mathrm{H}, \mathrm{R}^{3}=\mathrm{R}^{4}=\mathrm{OMe}, \mathrm{R}^{5}=\mathrm{H}$

q) $\mathrm{R}^{1}=\mathrm{R}^{2}=\mathrm{OMe}, \mathrm{R}^{3}=\mathrm{R}^{4}=\mathrm{R}^{5}=\mathrm{H}$

r) $\mathrm{R}^{1}=\mathrm{R}^{2}=\mathrm{R}^{3}=\mathrm{R}^{4}=$ OMe, $\mathrm{R}^{5}=\mathrm{H}$

s) $\mathrm{R}^{1}=\mathrm{R}^{2}=\mathrm{R}^{3}=\mathrm{R}^{4}=\mathrm{H}, \mathrm{R}^{5}=\mathrm{OMe}$

Scheme 2. Synthesis of 2,3-diaryl-9H-xanthen-9-ones $\mathbf{5 a - c}, \mathbf{5} \mathbf{g}-\mathbf{s}$ and $\mathbf{1 2 n - \mathbf { s }}$. 
rearrangements of $10 \mathrm{a}-\mathbf{i}$ in the presence of potassium hydroxide in DMSO gave the desired 5-aryl-3-hydroxy-1-(2hydroxyaryl)penta-2,4-dien-1-ones $\mathbf{1 1 a - i}$ in good yields. ${ }^{[28]}$ The key step in the synthesis of the (E)-3-bromo-2-styryl$4 H$-chromen-4-ones $\mathbf{3 a}$ and $\mathbf{3} \mathbf{d}-\mathbf{k}$ involves treatment of ketones 11a-i with phenyltrimethylammonium tribromide (PTT) in THF at room temperature. The presence of this reactant promotes a one-pot transformation, involving ketone $\alpha$-bromination ${ }^{[29]}$ followed by cyclisation to afford, after careful column chromatographic purification, the 3bromo-2-styryl-4H-chromen-4-ones $\mathbf{3 a}$ and $\mathbf{3 d}-\mathbf{k}$.

An extensive optimisation study on Heck reactions between the (E)-3-bromo-2-styryl-4H-chromen-4-ones 3a and $\mathbf{3 d}-\mathbf{k}$ and the styrenes $\mathbf{4 a}-\mathbf{c}$ was carried out, with investigation of the amounts of base and styrene and the reaction time and temperature. These reactions afforded the desired 2,3-diaryl-9H-xanthen-9-ones $\mathbf{5 a - c}$ and $\mathbf{5} \mathbf{g}-\mathbf{s}$, the 2,3-diaryl3,4-dihydro-9H-xanthen-9-one intermediates $\mathbf{6 a}-\mathbf{c}$ and $\mathbf{6 g}-\mathbf{-}$ and also the 6,7-diaryl-1-hydroxy-9H-xanthen-9-ones $\mathbf{1 2 n}$ $\mathbf{s}$, in the cases of compounds $\mathbf{3 h}-\mathbf{k}$, each bearing a 5-methoxy group from which the methyl group was cleaved off during the reaction. The yields of each of these compounds changed according to the experimental conditions used (see Experimental Section, Table 2 and Table 3).
Table 3. Best experimental conditions ( $T$ in all cases: reflux temp.) for the synthesis of 2,3-diaryl-9H-xanthen-9-ones $\mathbf{5 g}$-s, 2,3-diaryl3,4-dihydro-9H-xanthen-9-ones $\mathbf{6 g}-\mathbf{s}$ and 1-hydroxy-6,7-diaryl-9Hxanthen-9-ones $\mathbf{1 2 n}-\mathbf{s}$.

\begin{tabular}{|c|c|c|c|c|c|c|c|c|}
\hline & $t$ & $\begin{array}{c}\% \\
\text { Yield } \\
\mathbf{5}\end{array}$ & $\begin{array}{c}\% \\
\text { Yield } \\
\mathbf{6}\end{array}$ & $\begin{array}{c}\% \\
\text { Yield } \\
\mathbf{1 2}\end{array}$ & {$[\mathrm{h}]$} & $\begin{array}{c}\% \\
\text { Yield } \\
\mathbf{5}\end{array}$ & $\begin{array}{c}\% \\
\text { Yield } \\
\mathbf{6}\end{array}$ & $\%$ Yield \\
\hline $\mathbf{g}$ & 9 & 60 & 11 & - & 6 & 49 & 43 & - \\
\hline $\mathbf{h}$ & 9 & 62 & - & - & 3 & 46 & 10 & - \\
\hline $\mathbf{i}$ & 9 & 28 & - & - & 3 & 21 & 4 & - \\
\hline $\mathbf{j}$ & 9 & 62 & 3 & - & 6 & 41 & 13 & - \\
\hline $\mathbf{k}$ & 9 & 80 & - & - & 6 & 46 & 21 & - \\
\hline I & 3 & 70 & - & - & 9 & 55 & 6 & - \\
\hline $\mathbf{m}$ & 6 & 47 & 17 & - & 3 & 33 & 21 & - \\
\hline $\mathbf{n}$ & 9 & 58 & - & - & 6 & 47 & 12 & 12 \\
\hline $\mathbf{0}$ & 9 & 24 & - & 37 & 3 & 16 & 43 & 2 \\
\hline $\mathbf{p}$ & 6 & 22 & 10 & 3 & 3 & 22 & 5 & 2 \\
\hline $\mathbf{q}$ & 9 & 50 & - & 1 & 6 & 43 & 17 & 34 \\
\hline $\mathbf{r}$ & 9 & 43 & - & 11 & 3 & 31 & 13 & 5 \\
\hline $\mathbf{S}$ & 6 & 42 & - & 2 & 3 & 21 & 46 & 1 \\
\hline
\end{tabular}

Finally, to afford the desired polyhydroxylated 2,3-diaryl$9 H$-xanthen-9-ones $\mathbf{1 3 b}$-s it was necessary to cleave the benzyl and methyl groups of the 2,3-diaryl-9H-xanthen-9-ones 5b-s. Boron tribromide in dry dichloromethane was used as deprotecting agent for simultaneous cleavage of both pro-

Table 2. Best experimental conditions for the synthesis of the 2,3-diaryl-9H-xanthen-9-ones 5a-f and the 2,3-diaryl-3,4-dihydro-9Hxanthen-9-ones $\mathbf{6 a}-\mathbf{f}$.

\begin{tabular}{|c|c|c|c|c|c|c|c|c|}
\hline & $\begin{array}{l}\text { Time } \\
{[\mathrm{h}]}\end{array}$ & $\begin{array}{c}\text { Temperature } \\
{\left[{ }^{\circ} \mathrm{C}\right]}\end{array}$ & $\%$ Yield 5> & $\%$ Yield 6 & $\begin{array}{c}\text { Time } \\
{[\mathrm{h}]}\end{array}$ & $\begin{array}{c}\text { Temperature } \\
{\left[{ }^{\circ} \mathrm{C}\right]}\end{array}$ & $\%$ Yield $\mathbf{5}$ & $\%$ Yield 6 \\
\hline a & 9 & $160^{[\mathrm{a}]}$ & 56 & - & 9 & 160 & 54 & 9 \\
\hline $\begin{array}{l}a \\
b\end{array}$ & 3 & 160 & 66 & 14 & 3 & $160^{[\mathrm{a}]}$ & 58 & - \\
\hline c & 12 & 160 & 45 & $\begin{array}{l}14 \\
-\end{array}$ & 12 & $160^{[\mathrm{a}]}$ & 44 & 6 \\
\hline d & 6 & reflux & 51 & - & 3 & reflux & 46 & 11 \\
\hline e & 6 & reflux ${ }^{[\mathrm{a}]}$ & 66 & - & 3 & reflux & 25 & 27 \\
\hline f & 12 & reflux & 39 & 27 & 3 & reflux & 36 & 28 \\
\hline
\end{tabular}

[a] $\mathrm{Et}_{3} \mathrm{~N}$ (4 equiv.).<smiles>[Y]c1ccc(-c2cc3c(=O)c4c([Y2])cc([Y])cc4oc3cc2-c2ccc([R])c([Y])c2)c([Y])c1</smiles>

13b-s

b) $\mathrm{R}^{7}=\mathrm{R}^{8}=\mathrm{H}, \mathrm{R}^{9}=\mathrm{OH}, \mathrm{R}^{10}=\mathrm{R}^{11}=\mathrm{R}^{12}=\mathrm{H}$

c) $\mathrm{R}^{7}=\mathrm{R}^{8}=\mathrm{H}, \mathrm{R}^{9}=\mathrm{R}^{10}=\mathrm{OH}, \mathrm{R}^{11}=\mathrm{R}^{12}=\mathrm{H}$

d,g) $\mathrm{R}^{7}=\mathrm{OH}, \mathrm{R}^{8}=\mathrm{R}^{9}=\mathrm{R}^{10}=\mathrm{R}^{11}=\mathrm{R}^{12}=\mathrm{H}$

e,h) $\mathrm{R}^{7}=\mathrm{OH}, \mathrm{R}^{8}=\mathrm{H}, \mathrm{R}^{9}=\mathrm{OH}, \mathrm{R}^{10}=\mathrm{R}^{11}=\mathrm{R}^{12}=\mathrm{H}$

f,i) $\mathrm{R}^{7}=\mathrm{OH}, \mathrm{R}^{9}=\mathrm{H}, \mathrm{R}^{10}=\mathrm{R}^{4}=\mathrm{OH}, \mathrm{R}^{11}=\mathrm{R}^{12}=\mathrm{H}$

j) $\mathrm{R}^{7}=\mathrm{R}^{8}=\mathrm{OH}, \mathrm{R}^{9}=\mathrm{R}^{10}=\mathrm{R}^{11}=\mathrm{R}^{12}=\mathrm{H}$

k) $\mathrm{R}^{7}=\mathrm{R}^{8}=\mathrm{R}^{9}=\mathrm{OH}, \mathrm{R}^{10}=\mathrm{R}^{11}=\mathrm{R}^{12}=\mathrm{H}$

l) $\mathrm{R}^{7}=\mathrm{R}^{8}=\mathrm{R}^{9}=\mathrm{R}^{10}=\mathrm{OH}, \mathrm{R}^{11}=\mathrm{R}^{12}=\mathrm{H}$

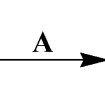<smiles>[R]c1cc([R])c2c(=O)c3cc(-c4ccc([R])c([R])c4)c(-c4ccc([R])c([R])c4)cc3oc2c1</smiles>

A: $\mathrm{CH}_{2} \mathrm{Cl}_{2}, \mathrm{BBr}_{3},-78{ }^{\circ} \mathrm{C}$ to room temp., $\mathrm{N}_{2}$

m) $\mathrm{R}^{7}=\mathrm{R}^{8}=\mathrm{R}^{9}=\mathrm{R}^{10}=\mathrm{H}, \mathrm{R}^{11}=\mathrm{OH}, \mathrm{R}^{12}=\mathrm{H}$

n) $\mathrm{R}^{7}=\mathrm{R}^{8}=\mathrm{R}^{9}=\mathrm{R}^{10}=\mathrm{R}^{11}=\mathrm{H}, \mathrm{R}^{12}=\mathrm{OH}$

о) $\mathrm{R}^{7}=\mathrm{R}^{8}=\mathrm{H}, \mathrm{R}^{9}=\mathrm{OH}, \mathrm{R}^{10}=\mathrm{R}^{11}=\mathrm{H}, \mathrm{R}^{12}=\mathrm{OH}$

q) $\mathrm{R}^{7}=\mathrm{R}^{8}=\mathrm{OH}, \mathrm{R}^{9}=\mathrm{R}^{10}=\mathrm{R}^{11}=\mathrm{H}, \mathrm{R}^{12}=\mathrm{OH}$

r) $\mathrm{R}^{7}=\mathrm{R}^{8}=\mathrm{R}^{9}=\mathrm{R}^{10}=\mathrm{OH}, \mathrm{R}^{11}=\mathrm{H}, \mathrm{R}^{12}=\mathrm{OH}$

s) $\mathrm{R}^{7}=\mathrm{R}^{8}=\mathrm{R}^{9}=\mathrm{R}^{10}=\mathrm{H}, \mathrm{R}^{11}=\mathrm{R}^{12}=\mathrm{OH}$

Scheme 3. Cleavage of the hydroxy protecting groups. 
<smiles>[R]c1ccc(/C=C/c2oc3ccccc3c(=O)c2Br)cc1[R]</smiles>

3a-c<smiles>[R]c1ccc(C=Cc2c(C)oc3ccccc3c2=O)cc1[R]</smiles>

7a-c<smiles>[R]c1ccc(/C=C/c2c(/C=C/c3ccccc3)oc3ccccc3c2=O)cc1[R]</smiles><smiles>[R]c1ccc(C(=O)/C=C(O)/C=C/c2ccc([R])c([R])c2)c(O)c1</smiles>

11a-i<smiles>[R]c1ccc(-c2cc3oc4ccccc4c(=O)c3cc2-c2ccc([R])c([R])c2)cc1[R]</smiles>

5a-f<smiles>[R]c1cc([R])c2c(=O)c3cc(-c4cc([R])c([R])c([R])c4)c(-c4ccc([R])c([R])c4)cc3oc2c1</smiles>

Figure 1. Structures and numbering system used for $(E)$-3-bromo-2-styryl-4H-chromen-4-ones 3a-c, $(E)$-2-methyl-3-styryl-4H-chromen4-ones 7a-c, $(E, E)$-2,3-distyryl-4H-chromen-4-ones 8a-c, $9 H$-xanthen-9-ones 5a-f, 5n-s, 12n-s and 13n-s and 5-aryl-3-hydroxy-1-(2-hydroxyphenyl)penta-2,4-dien-1-ones 11a-i. (We applied the recommended IUPAC rules to establish the names of all compounds, but preferred the numbering systems indicated in Figure 1 and retained this atom numbering of each compound for reasons of better comparability of NMR data. For example, according to IUPAC nomenclature the $9 H$-xanthen-9-ones $\mathbf{5 a}-\mathbf{f}$ and $\mathbf{5 n}-\mathbf{s}, \mathbf{1 2 n}-\mathbf{s}$ and $\mathbf{1 3 n}-\mathbf{s}$ must have different numbering, but we kept the same numbering for consistancy of NMR signal assignments).

tecting groups. ${ }^{[30,31]}$ The polyhydroxy-2,3-diaryl-9Hxanthen-9-ones 13b-s were obtained in moderate to good yields (Scheme 3, Figure 1).

\section{Nuclear Magnetic Resonance Spectroscopy}

The most important features in the ${ }^{1} \mathrm{H}$ NMR spectra of the 5-aryl-3-hydroxy-1-(2-hydroxyaryl)penta-2,4-dien-1ones 11a-i are, in each case: i) the singlets at $\delta=6.17-6.80$ and $12.28-13.63 \mathrm{ppm}$, corresponding to $2-\mathrm{H}$ and $2^{\prime}-\mathrm{OH}$, respectively, and ii) a doublet at $\delta=14.51-15.01 \mathrm{ppm}$ with $J \approx 1 \mathrm{~Hz}$, due to long-range coupling with the 4-H proton, corresponding to the 3-hydroxy group. The high-frequency values of the last two signals are due to the deshielding effect of the hydrogen bonds of the hydroxy protons with a carbonyl group. NOESY correlations between the signal of 2-H and those of 4-H and $6^{\prime}-\mathrm{H}$ (for 11a-e), and of 4-H and $5-\mathrm{H}$ with $2^{\prime \prime}, 6^{\prime \prime}-\mathrm{H}$, together with the coupling constant of $J_{\mathrm{H} 4, \mathrm{H} 5}=15-16 \mathrm{~Hz}$ indicate that the compounds of this type have planar $(Z)-s-(Z)-(E)$ configurations (Figure 2$)$.
The ${ }^{1} \mathrm{H}$ NMR spectra of the $(E)$-3-bromo-2-styryl-4Hchromen-4-ones $\mathbf{3 a}-\mathbf{k}$ each revealed the presence of two doublets, corresponding to the vinylic protons $\alpha-\mathrm{H}$ and $\beta$ $\mathrm{H}[\delta=7.20-7.46$ and $7.58-7.73 \mathrm{ppm}$, respectively] in a trans configuration $(J \approx 16.0 \mathrm{~Hz})$. The high-frequency values assigned to the resonances of $\beta-\mathrm{H}$ and $\mathrm{C}-\beta(\delta=138.1-$ $139.6 \mathrm{ppm})$ relative to the resonance of $\alpha-\mathrm{H}$ and $\mathrm{C}-\alpha(\delta=$ 116.9-119.3 ppm) are due to the mesomeric deshielding effect of the carbonyl group. HMBC spectra allowed the assignment of the quaternary carbons of the chromone moiety: C-2 and C-9 at $\delta=156.1-158.8$ and 154.8-158.4 ppm, respectively, C-3 at $\delta=108.9-116.9 \mathrm{ppm}$ and $\mathrm{C}-10$ at $\delta=$ 107.5-122.1 ppm.

An important feature in the ${ }^{1} \mathrm{H}$ NMR spectra of the $(E)$ 2-methyl-3-styryl-4H-chromen-4-ones $\mathbf{7 a - c}$ is the presence in each case of a singlet corresponding to the $2-\mathrm{CH}_{3}$ protons at $\delta=2.61-2.62 \mathrm{ppm}$, and doublets corresponding to the olefinic protons $\alpha-\mathrm{H}(\delta=6.83-6.97 \mathrm{ppm})$ and $\beta-\mathrm{H}(\delta=$ $7.59-7.71 \mathrm{ppm})$ in a trans configuration $(J=16.3 \mathrm{~Hz})$. The difference in the chemical shifts is due to the deshielding

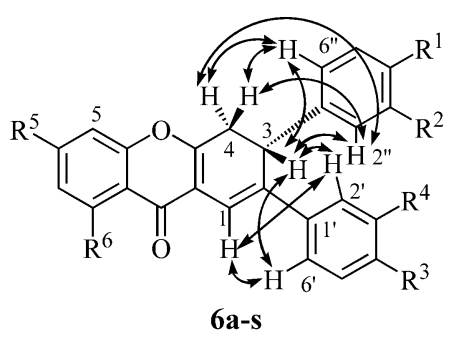

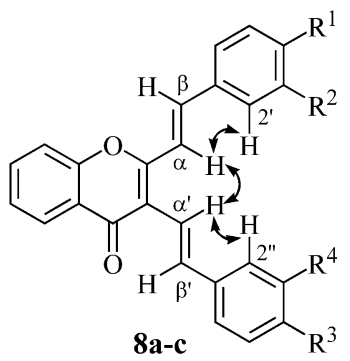

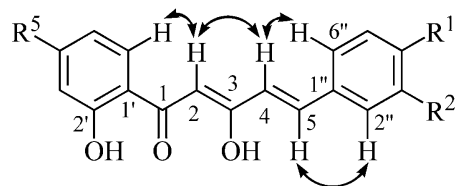

$11 \mathrm{a}-\mathbf{i}$

Figure 2. Main NOE cross peaks found in the NOESY spectra of the 2,3-diaryl-3,4-dihydro-9H-xanthen-9-ones 6a-s, the (E,E)-2,3distyryl-4H-chromen-4-ones 8a-c and the 5-aryl-3-hydroxy-1-(2-hydroxyphenyl)penta-2,4-dien-1-ones 11a-i. 

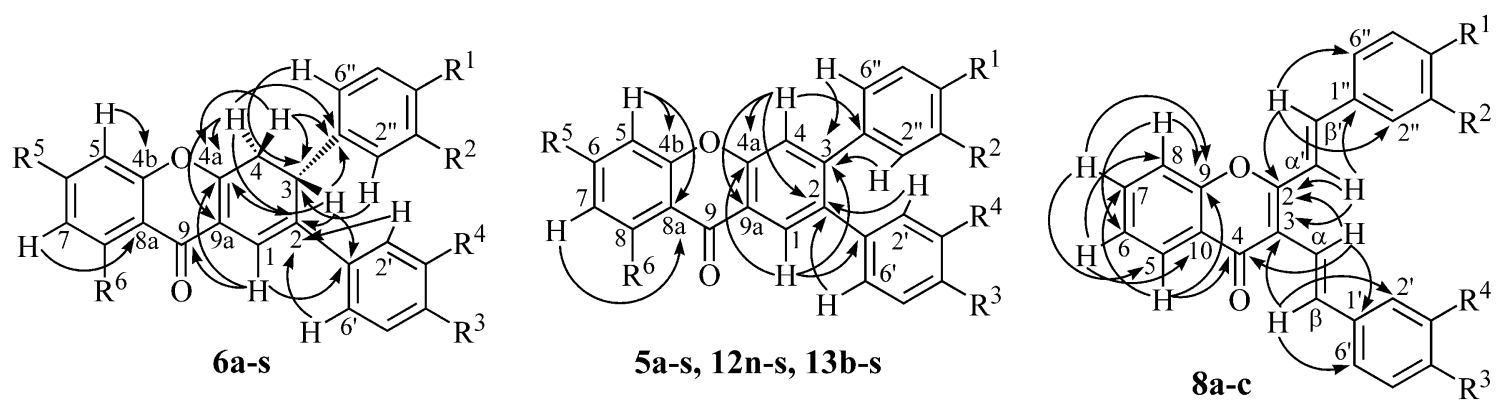

Figure 3. Important connectivities found in the HMBC spectra of the 2,3-diaryl-9H-xanthen-9-ones $\mathbf{5 a}-\mathbf{s}, \mathbf{1 2 n}-\mathbf{s}$ and $\mathbf{1 3 b}-\mathbf{s}$, the 2,3-diaryl3,4-dihydro-9H-xanthen-9-ones 6a-s and the $(E, E)$-2,3-distyryl-4H-chromen-4-ones 8a-c.

effect of the chromone moiety, $\beta-\mathrm{H}$ being the more deshielded. NOESY correlations between the protons of the methyl group and the two protons $\alpha-\mathrm{H}$ and $\beta-\mathrm{H}$, indicate free rotation around the $\mathrm{C}-3$ and $\mathrm{C}-\alpha$ bonds. The ${ }^{13} \mathrm{C}$ NMR spectra of the $(E)$-2-methyl-3-styryl-4H-chromen-4-ones $7 \mathbf{a}-\mathbf{c}$ each present the characteristic signals corresponding to the 2-methyl carbon at $\delta=19.3-19.4 \mathrm{ppm}$, to $\mathrm{C}-2$ at $\delta=$ $163.3-163.8 \mathrm{ppm}$ and to the carbonyl carbon at $\delta=176.9$ $177.0 \mathrm{ppm}$.

The NMR spectra of the $(E, E)$-2,3-distyryl-4H-chromen4-ones $\mathbf{8 a}-\mathbf{c}$ are not easy to interpret because of the symmetry of the molecules and the fact that all the signals are in the aromatic region, except for the signals corresponding to $5-\mathrm{H}$, which each appear as a double doublet at the highest frequency value ( $\delta=8.22-8.25 \mathrm{ppm})$. The assignment of the two $\mathrm{AB}$ spin-systems, due in each case to the resonances of $\alpha-\mathrm{H}(\delta=7.37-7.42 \mathrm{ppm}), \beta-\mathrm{H}(\delta=7.67-7.71 \mathrm{ppm}), \alpha^{\prime}-$ $\mathrm{H}(\delta=7.11-7.21 \mathrm{ppm})$ and $\beta^{\prime}-\mathrm{H}(\delta=7.27-7.43 \mathrm{ppm})$, was made on the basis of the connectivities found in the HMBC spectra. The correlation observed in these spectra between the signal of the carbonyl carbon and the doublet at $\delta=$ 7.11-7.21 ppm identifies $\alpha^{\prime}-\mathrm{H}$ (Figure 3 ). The coupling constants of the vinylic systems $(J \approx 16 \mathrm{~Hz})$ indicate their trans configurations, whereas the NOE cross peaks between the signals of $\alpha-\mathrm{H}$ and $\alpha^{\prime}-\mathrm{H}$, as well as those of $\alpha-\mathrm{H}$ and $2^{\prime}, 6^{\prime}-\mathrm{H}$ and also of $\alpha^{\prime}-\mathrm{H}$ and $2^{\prime \prime}, 6^{\prime \prime}-\mathrm{H}$, support the conformations presented in Figure 2 for compounds 8a-c.

In the ${ }^{1} \mathrm{H}$ NMR spectra of all the synthesised 2,3-diaryl$9 H$-xanthen-9-ones $\mathbf{5 a}-\mathbf{s}, \mathbf{1 2 n}-\mathbf{s}$ and $\mathbf{1 3 b}-\mathbf{s}$, the signals corresponding to $1-\mathrm{H}$ and $4-\mathrm{H}$ appear as singlets at $\delta=7.91-$ 8.38 and $7.43-7.65 \mathrm{ppm}$, respectively; the high-frequency values of the $1-\mathrm{H}$ protons are due to the anisotropic and mesomeric deshielding effects of the carbonyl groups. In the case of the xanthones $\mathbf{1 2 n}-\mathbf{s}$ and $\mathbf{1 3 n}-\mathbf{s}$ it was also possible to identify the singlets corresponding to the resonances of the 8-hydroxy groups at $\delta=12.48-12.92 \mathrm{ppm}$. The highfrequency values of these resonances are due to intramolecular hydrogen bonding of the hydroxy protons with the carbonyl groups. For compounds $\mathbf{1 3 b}-\mathbf{n}$, the signals corresponding to the proton resonance of the hydroxy groups also appear as singlets, at high-frequency values, with chemical shifts higher than $8.9 \mathrm{ppm}$.

The correlations found in the HMBC spectra provided unequivocal support for the structures of the 2,3-diaryl-9H- xanthen-9-ones $\mathbf{5 a}-\mathbf{s}, \mathbf{1 2 n}-\mathbf{s}$ and $\mathbf{1 3 b}-\mathbf{s}$, allowing the assignment of the quaternary carbons of the xanthone cores. The main connectivities are: i) $4-\mathrm{H}$ and $2^{\prime}, 6^{\prime}-\mathrm{H}$ with $\mathrm{C}-2$, ii) $1-$ $\mathrm{H}$ and $2^{\prime \prime}, 6^{\prime \prime}-\mathrm{H}$ with $\mathrm{C}-3$, iii) $1-\mathrm{H}$ and $4-\mathrm{H}$ with $\mathrm{C}-4 \mathrm{a}$, iv) 5$\mathrm{H}$ and 6-H with $\mathrm{C}-4 \mathrm{~b}$, v) $5-\mathrm{H}$ and 7-H with $\mathrm{C}-8 \mathrm{a}$, and vi) 4$\mathrm{H}$ with carbon C-9a (Figure 3).

The ${ }^{1} \mathrm{H}$ NMR spectra of the 2,3-diaryl-3,4-dihydro- $9 \mathrm{H}$ xanthen-9-ones $\mathbf{6 a}-\mathbf{s}$ in each case revealed the presence of the aliphatic protons $\mathrm{H}-3$ and H-4. The signal corresponding to 3-H appears as a doublet or a double doublet, at $\delta$ $=4.22-4.31 \mathrm{ppm}$ and at a higher-frequency value than that of $\mathrm{H}-4$. From the coupling constants, the stereochemistry of $4_{\text {cis }}-\mathrm{H}(J=8.3-9.1 \mathrm{~Hz}, \delta=3.57-3.67 \mathrm{ppm})$ and $4_{\text {trans }}-\mathrm{H}$ $(J=1.3-1.6 \mathrm{~Hz}, \delta=2.89-3.01 \mathrm{ppm})$ is related to $3-\mathrm{H}$. Another main feature in each ${ }^{1} \mathrm{H}$ NMR spectrum is the presence, in the aromatic region, of a singlet at $\delta=7.44$ $7.56 \mathrm{ppm}$ corresponding to $1-\mathrm{H}$. The correlations found in the HMBC spectrum between this singlet $(1-\mathrm{H})$ and the signal of the carbonyl carbon and also between the signal of $3-\mathrm{H}$ with those of carbons $\mathrm{C}-2^{\prime \prime}, 6^{\prime \prime}$, supports the presence of the 3,4-dihydro-9H-xanthen-9-one moiety (Figure 3). This structure was also confirmed by the correlations found in the NOESY spectrum, mainly those indicating close proximity of $1-\mathrm{H}$ to $2^{\prime}, 6^{\prime}-\mathrm{H}$, of $3-\mathrm{H}$ to $2^{\prime}, 6^{\prime}-\mathrm{H}$ and $2^{\prime \prime} 6^{\prime \prime}-$ $\mathrm{H}$ and of $4_{\text {cis }}-\mathrm{H}$ and 4 trans $^{-\mathrm{H}}$ to $2^{\prime \prime}, 6^{\prime \prime}$-H (Figure 3).

\section{Conclusions}

We have reported two different routes for the synthesis of hydroxylated 2,3-diaryl-9H-xanthen-9-ones. One of them involves Heck reactions between 3-bromo-2-methyl-4Hchromen-4-one and styrenes, followed by aldol condensations of the obtained compounds with benzaldehyde to gave 2,3-distyryl-4H-chromen-4-ones, which gave the desired 2,3diaryl-9H-xanthen-9-ones after electrocyclisation and oxidation processes. In the second route, the 2,3-diaryl-9Hxanthen-9-ones were obtained through Heck reactions between 3-bromo-2-styryl-4H-chromen-4-ones and styrenes. The 3-bromo-2-styryl-4H-chromen-4-ones were obtained either through aldol condensations between 3-bromo-2methyl-4H-chromen-4-one and benzaldehydes or through Baker-Venkataraman rearrangements of 2-acetylphenyl cinnamates followed by one-pot bromination/cyclisation 
with phenyltrimethylammonium tribromide. The cleavage of the methyl and benzyl protecting groups with boron tribromide gave the desired hydroxylated 2,3-diaryl-9Hxanthen-9-ones.

\section{Experimental Section}

General Remarks: Melting points were measured in a Reichert Thermovar apparatus fitted with a microscope and are uncorrected. NMR spectra were recorded on a Bruker Avance 300 spectrometer $\left(300.13 \mathrm{MHz}\right.$ for ${ }^{1} \mathrm{H}$ and $75.47 \mathrm{MHz}$ for $\left.{ }^{13} \mathrm{C}\right)$, in $\mathrm{CDCl}_{3}$ as solvent if not stated otherwise. Chemical shifts $(\delta)$ are reported in ppm and coupling constants $(J)$ in $\mathrm{Hz}$; the internal standard was TMS. Unequivocal ${ }^{13} \mathrm{C}$ assignments were made with the aid of $2 \mathrm{D}$ $g \mathrm{HSQC}$ and $g \mathrm{HMBC}$ (delays for one-bond and long-range $J \mathrm{C} / \mathrm{H}$ couplings were optimised for 145 and $7 \mathrm{~Hz}$, respectively) experiments. Positive-ion ESI mass spectra were acquired with a QTOF 2 instrument [dilution of $1 \mu \mathrm{L}$ of the sample in chloroform solution (ca. $10^{-5} \mathrm{M}$ ) in $200 \mu \mathrm{L}$ of $0.1 \%$ trifluoroacetic acid/methanol solution. Nitrogen was used as nebuliser gas and argon as collision gas. The needle voltage was set at $3000 \mathrm{~V}$, with the ion source at $80^{\circ} \mathrm{C}$ and the desolvation temperature at $150^{\circ} \mathrm{C}$. The cone voltage was $35 \mathrm{~V}]$. Other low- and high-resolution mass spectra $(\mathrm{EI}, 70 \mathrm{eV}$ ) were measured with VG Autospec $\mathrm{Q}$ and $\mathrm{M}$ spectrometers. Elemental analyses were obtained with a LECO 932 CHNS analyser. Preparative thin-layer chromatography was performed with Merck silica gel $\left(60 \mathrm{DGF}_{254}\right)$. Column chromatography was performed with Merck silica gel (60, 70-230 mesh). All chemicals and solvents used were obtained from commercial sources and used as received or dried by standard procedures.

Synthesis of (E)-3-Bromo-2-styryl-4H-chromen-4-ones 3a and 3b: Sodium $(0.4 \mathrm{~g}, 16.7 \mathrm{mmol})$ was gradually added to dry methanol $(20 \mathrm{~mL})$ and the mixture was stirred until the reaction mixture had returned to room temperature. 3-Bromo-2-methyl-4H-chromen-4one $(1,1.0 \mathrm{~g}, 4.2 \mathrm{mmol})$ and the appropriate benzaldehyde $2 \mathrm{a}$ or $\mathbf{2 b}(5.0 \mathrm{mmol})$ were added and the reaction mixture was allowed to stand at room temperature for $48 \mathrm{~h}$. After this period, the solution was poured into ice $(100 \mathrm{~g})$ and water $(100 \mathrm{~mL})$ and the $\mathrm{pH}$ was adjusted to 4 with hydrochloric acid. The yellow solid was removed by filtration, taken up in dichloromethane and purified by silica gel column chromatography with dichloromethane as eluent. The solvent was removed to dryness and the residue was recrystallised from ethanol to give the $(E)$-3-bromo-2-styryl-4H-chromen-4-ones $3 \mathbf{a}$ or $\mathbf{3 b}$.

(E)-3-Bromo-2-(2-phenylvinyl)-4H-chromen-4-one (3a): Yield $1.42 \mathrm{~g}$ (87\%); m.p. $167-169{ }^{\circ} \mathrm{C} .{ }^{1} \mathrm{H}$ NMR: $\delta=7.40-7.49$ (m, $4 \mathrm{H}, 6-\mathrm{H}$, $\left.3^{\prime}, 4^{\prime}, 5^{\prime}-\mathrm{H}\right), 7.51(\mathrm{~d}, J=16.0 \mathrm{~Hz}, 1 \mathrm{H}, \alpha-\mathrm{H}), 7.56(\mathrm{~d}, J=7.9 \mathrm{~Hz}, 1$ H, 8-H), 7.64-7.67 (m, 2 H, 2', 6'-H), $7.72(\mathrm{dt}, J=7.9,1.7 \mathrm{~Hz}, 1$ $\mathrm{H}$ and $7-\mathrm{H}), 7.73(\mathrm{~d}, J=16.0 \mathrm{~Hz}, 1 \mathrm{H}, \beta-\mathrm{H}), 8.25(\mathrm{dd}, J=8.0$, $1.7 \mathrm{~Hz}, 1 \mathrm{H}, 5-\mathrm{H}) \mathrm{ppm} .{ }^{13} \mathrm{C}$ NMR: $\delta=109.8(\mathrm{C}-3), 117.5(\mathrm{C}-8)$, $119.2(\mathrm{C}-\alpha), 122.1$ (C-10), 125.4 (C-6), 126.4 (C-5), $128.1\left(\mathrm{C}-2^{\prime}, 6^{\prime}\right)$, $129.1\left(\mathrm{C}-3^{\prime}, 5^{\prime}\right), 130.3\left(\mathrm{C}-4^{\prime}\right), 134.1$ (C-7), $134.9\left(\mathrm{C}-1^{\prime}\right), 139.6(\mathrm{C}-\beta)$, 154.9 (C-9), 158.4 (C-2), 172.8 (C-4) ppm. EI-MS: $m / z(\%)=328$ $[\mathrm{M}]^{+\cdot}\left({ }^{81} \mathrm{Br}, 22\right), 326[\mathrm{M}]^{+\cdot}\left({ }^{79} \mathrm{Br}, 22\right), 282(10), 247(100), 218(12)$, 191 (11), 189 (12), 165 (4), 127 (24), 109 (7), 101 (3), 92 (10), 77 (9), 63 (6), 51 (4). $\mathrm{C}_{17} \mathrm{H}_{11} \mathrm{BrO}_{2}$ (327.13): calcd. $\mathrm{C}$ 62.41, H 3.39; found C 62.49, H 3.42 .

(E)-4'-Benzyloxy-3-bromo-2-(2-phenylvinyl)-4H-chromen-4-one (3b): This compound was shown to possess spectroscopic and analytical data identical to those previously reported. ${ }^{[26]}$

Synthesis of the 2,3-Diaryl-9H-xanthen-9-ones 5a-f: The appropriate styrene (4a-c, $3 \mathrm{mmol}$ for styrene $\mathbf{4 a}$ and $0.8 \mathrm{mmol}$ for styrenes 4b or 4c) was added to a mixture of the appropriate 3-bromo-2styryl-4H-chromen-4-one (3a or $\mathbf{3 b}, 0.6 \mathrm{mmol}$ ), triphenylphosphane $(15.7 \mathrm{mg}, 0.06 \mathrm{mmol})$, tetrakis(triphenylphosphane)palla$\operatorname{dium}(0) \quad(34.7 \mathrm{mg}, \quad 0.03 \mathrm{mmol})$ and triethylamine $(83.6 \mu \mathrm{L}$, $0.6 \mathrm{mmol})$ in $\mathrm{N}$-methyl-2-pyrrolidin-1-one $(10 \mathrm{~mL})$. The mixture was stirred under different time and temperature conditions according to the substitution on the compounds (Table 2). The mixture was then poured into water $(20 \mathrm{~mL})$ and ice $(10 \mathrm{~g})$ and extracted with diethyl ether $(4 \times 25 \mathrm{~mL})$ and dried with anhydrous sodium sulfate. The residue was concentrated, taken up in dichloromethane $(5 \mathrm{~mL})$ and purified by thin layer chromatography (eluent: dichloromethane/light petroleum, 7:3). The major spot with higher $R_{\mathrm{f}}$ value corresponds to the 2,3-diaryl-9H-xanthen-9-one (5a-f) and a minor spot with lower $R_{\mathrm{f}}$ corresponds to the 2,3-diaryl-3,4dihydro-9H-xanthen-9-one (6a-f). The 2,3-diaryl-9H-xanthen-9ones $\mathbf{5} \mathbf{a}-\mathbf{f}$ were recrystallised from ethanol and obtained in moderate to good yields.

2,3-Diaryl-9H-xanthen-9-ones 5a and 5b and 2,3-Diphenyl-3,4-dihydro-9H-xanthen-9-ones $6 \mathrm{a}$ and 6f: These compounds were shown to possess spectroscopic and analytical data identical to those previously reported. ${ }^{[26]}$

2-(4-Methoxyphenyl)-3-phenyl-3,4-dihydro-9H-xanthen-9-one (6b): M.p. $153-156{ }^{\circ} \mathrm{C} .{ }^{1} \mathrm{H}$ NMR: $\delta=2.99(\mathrm{dd}, J=17.3,1.3 \mathrm{~Hz}, 1 \mathrm{H}$, $\left.4_{\text {trans }}-\mathrm{H}\right), 3.64\left(\mathrm{dd}, J=17.3,9.0 \mathrm{~Hz}, 1 \mathrm{H}, 4_{\text {cis }}-\mathrm{H}\right), 3.77\left(\mathrm{~s}, 3 \mathrm{H}, 4^{\prime}\right.$ $\left.\mathrm{OCH}_{3}\right), 4.27(\mathrm{dd}, J=9.0,1.3 \mathrm{~Hz}, 1 \mathrm{H}, 3-\mathrm{H}), 6.81(\mathrm{~d}, J=8.9 \mathrm{~Hz}$, $\left.2 \mathrm{H}, 3^{\prime}, 5^{\prime}-\mathrm{H}\right), 7.15-7.40$ (m, $\left.7 \mathrm{H}, 5,7,2^{\prime \prime}, 3^{\prime \prime}, 4^{\prime \prime}, 5^{\prime \prime}, 6^{\prime \prime}-\mathrm{H}\right), 7.43$ (d, $\left.J=8.9 \mathrm{~Hz}, 2 \mathrm{H}, 2^{\prime}, 6^{\prime}-\mathrm{H}\right), 7.46(\mathrm{~s}, 1 \mathrm{H}, 1-\mathrm{H}), 7.58(\mathrm{dt}, J=7.8$, $1.6 \mathrm{~Hz}, 1 \mathrm{H}, 6-\mathrm{H}), 8.28(\mathrm{dd}, J=7.8,1.6 \mathrm{~Hz}, 1 \mathrm{H}, 8-\mathrm{H}) \mathrm{ppm} .{ }^{13} \mathrm{C}$ NMR: $\delta=36.6(\mathrm{C}-4), 41.6(\mathrm{C}-3), 55.2\left(4^{\prime}-\mathrm{OCH}_{3}\right), 113.8\left(\mathrm{C}-3^{\prime}, 5^{\prime}\right)$, 114.8 (C-1), 117.0 (C-9a), 118.0 (C-5), 123.8 (C-8a), 125.0 (C-7), 126.1 (C-8), $126.8\left(\mathrm{C}-2^{\prime}, 6^{\prime}\right), 127.1\left(\mathrm{C}-4^{\prime \prime}\right), 127.3\left(\mathrm{C}-2^{\prime \prime}, 6^{\prime \prime}\right), 128.9$ $\left(\mathrm{C}-3^{\prime \prime}, 5^{\prime \prime}\right), 131.5\left(\mathrm{C}-1^{\prime}\right), 132.9$ (C-6), 134.9 (C-2), 140.8 (C-1' ), 155.8 (C-4b), 159.1 (C-4'), 162.0 (C-4a), 174.2 (C-9) ppm. EI-MS: $\mathrm{m} / \mathrm{z}(\%)=380(100)[\mathrm{M}]^{+\cdot}, 379(18), 378(31), 365(6), 347(3), 303$ (23), 287 (6), 273 (16), 260 (10), 215 (9), 202 (6), 190 (6), 165 (3), 139 (3), 121 (14), 108 (3), 92 (5), 77 (6), 65 (4), 51 (4). HRMS (EI): calcd. for $\mathrm{C}_{26} \mathrm{H}_{20} \mathrm{O}_{3}[\mathrm{M}]^{+\cdot} 380.1412$; found 380.1412 .

2-(3,4-Dimethoxyphenyl)-3-phenyl-9H-xanthen-9-one (5c): M.p. 139-140 ${ }^{\circ} \mathrm{C} .{ }^{1} \mathrm{H}$ NMR: $\delta=3.56$ and $3.87\left(2 \times \mathrm{s}, 6 \mathrm{H}, 3^{\prime}, 4^{\prime}-\mathrm{OCH}_{3}\right)$, $6.54\left(\mathrm{~d}, J=1.9 \mathrm{~Hz}, 1 \mathrm{H}, 2^{\prime}-\mathrm{H}\right), 6.78-6.90\left(\mathrm{~m}, 2 \mathrm{H}, 5^{\prime} \mathrm{H}\right.$ and $\left.6^{\prime}-\mathrm{H}\right)$, 7.20-7.24 (m, 2 H, $\left.2^{\prime \prime}, 6^{\prime \prime}-\mathrm{H}\right), 7.26-7.32$ (m, $\left.3 \mathrm{H}, 3^{\prime \prime}, 4^{\prime \prime}, 5^{\prime \prime}-\mathrm{H}\right), 7.40$ (dt, $J=7.6,1.0 \mathrm{~Hz}, 1 \mathrm{H}, 7-\mathrm{H}), 7.52(\mathrm{~d}, J=8.2 \mathrm{~Hz}, 1 \mathrm{H}, 5-\mathrm{H}), 7.55$ (s, $1 \mathrm{H}, 4-\mathrm{H}), 7.74$ (ddd, $J=8.2,7.6,1.7 \mathrm{~Hz}, 1 \mathrm{H}, 6-\mathrm{H}$ ), 8.38 (dd, $J=7.6,1.7 \mathrm{~Hz}, 1 \mathrm{H}, 8-\mathrm{H}), 8.39(\mathrm{~s}, 1 \mathrm{H}, 1-\mathrm{H}) \mathrm{ppm} .{ }^{13} \mathrm{C} \mathrm{NMR}: \delta=$ 55.7 and $55.9\left(3^{\prime}, 4^{\prime}-\mathrm{OCH}_{3}\right), 110.7\left(\mathrm{C}-5^{\prime}\right), 113.4\left(\mathrm{C}-2^{\prime}\right), 118.0(\mathrm{C}-5)$, 119.5 (C-4), 120.7 (C-9a), 121.9 (C-8a), 122.1 (C-6'), 123.9 (C-7), 126.7 (C-8), $127.5\left(\mathrm{C}-4^{\prime \prime}\right), 128.7$ (C-1), $128.2\left(\mathrm{C}-3^{\prime \prime}{ }^{\prime}, 5^{\prime \prime}\right), 129.5$ (C$\left.2^{\prime \prime}, 6^{\prime \prime}\right), 132.2\left(\mathrm{C}-1^{\prime}\right), 134.8(\mathrm{C}-6), 136.7$ (C-2), $140.1\left(\mathrm{C}-1^{\prime \prime}\right), 147.6$ (C-3), 148.0 and 148.1 (C-3', C-4'), 155.0 (C-4a), 156.2 (C-4b), 177.0 (C-9) ppm. EI-MS: $m / z(\%)=408\left[\right.$ M $^{+*}(100), 393$ (9), 361 (8), 333 (8), 321 (7), 292 (4), 263 (3), 204 (4), 188 (3), 167 (4), 138 (2), 77 (1). $\mathrm{C}_{27} \mathrm{H}_{20} \mathrm{O}_{4}$ (408.45): calcd. for $\mathrm{C} 79.40, \mathrm{H} 4.94$; found $\mathrm{C}$ 79.64, H 4.83 .

2-(3,4-Dimethoxyphenyl)-3-phenyl-3,4-dihydro-9H-xanthen-9-one (6c): Yellowish oil. ${ }^{1} \mathrm{H}$ NMR: $\delta=3.00(\mathrm{dd}, J=17.3,1.5 \mathrm{~Hz}, 1 \mathrm{H}$, $\left.4_{\text {trans }}-\mathrm{H}\right), 3.65\left(\mathrm{dd}, J=17.3,9.1 \mathrm{~Hz}, 1 \mathrm{H}, 4_{\text {cis }}-\mathrm{H}\right), 3.84\left(\mathrm{~s}, 6 \mathrm{H}, 3^{\prime}, 4^{\prime}\right.$ $\left.\mathrm{OCH}_{3}\right), 4.29(\mathrm{dd}, J=9.1,1.5 \mathrm{~Hz}, 1 \mathrm{H}, 3-\mathrm{H}), 6.74(\mathrm{~d}, J=8.4 \mathrm{~Hz}$, $\left.1 \mathrm{H}, 5^{\prime}-\mathrm{H}\right), 6.96\left(\mathrm{dd}, J=8.4,2.2 \mathrm{~Hz}, 1 \mathrm{H}, 6^{\prime}-\mathrm{H}\right), 7.09$ (d, $J=$ $\left.2.2 \mathrm{~Hz}, 1 \mathrm{H}, 2^{\prime}-\mathrm{H}\right), 7.18-7.32$ (m, $\left.5 \mathrm{H}, 2^{\prime \prime}, 3^{\prime \prime}, 4^{\prime \prime}, 5^{\prime \prime}, 6^{\prime \prime}-\mathrm{H}\right), 7.33-$ 7.41 (m, 2 H, 5-H, 7-H), 7.46 (s, $1 \mathrm{H}, 1-\mathrm{H}), 7.59$ (dt, $J=7.8,1.6 \mathrm{~Hz}$, $1 \mathrm{H}, 6-\mathrm{H}), 8.28(\mathrm{dd}, J=8.0,1.6 \mathrm{~Hz}, 1 \mathrm{H}, 8-\mathrm{H}) \mathrm{ppm} .{ }^{13} \mathrm{C}$ NMR: $\delta$ $=36.5(\mathrm{C}-4), 41.8(\mathrm{C}-3), 55.8\left(3^{\prime}, 4^{\prime}-\mathrm{OCH}_{3}\right), 108.7\left(\mathrm{C}-2^{\prime}\right), 110.8(\mathrm{C}-$ 
5'), 115.0 (C-1), 116.9 (C-9a), 117.9 (C-5), 118.2 (C-6'), 123.8 (C8a), 125.0 (C-7), 126.1 (C-8), 127.1 (C-4" ), 127.2 (C-2' ,6 $\left.6^{\prime \prime}\right), 128.9$ (C-3' , 5' $), 131.9$ (C-1'), 133.0 (C-6), 135.1 (C-2), 140.9 (C-1' '), 148.70 and $148.72\left(\mathrm{C}-3^{\prime}, 4^{\prime}\right), 155.8$ (C-4b), 162.1 (C-4a), 174.2 (C9) ppm. EI-MS: $\mathrm{m} / \mathrm{z}=410[\mathrm{M}]^{+\cdot}(100), 409$ (8), 408 (20), 395 (7), 379 (4), 361 (3), 333 (15), 319 (4), 289 (4), 273 (13), 205 (5), 165 (12), 151 (6), 138 (5), 121 (9), 92 (4), 77 (5). HRMS (EI): calcd. for $\mathrm{C}_{27} \mathrm{H}_{22} \mathrm{O}_{4}[\mathrm{M}]^{+\cdot}$ 410.1518; found 410.1521 .

3-(4-Benzyloxyphenyl)-2-phenyl-9H-xanthen-9-one (5d): M.p. 182 $183{ }^{\circ} \mathrm{C} .{ }^{1} \mathrm{H}$ NMR: $\delta=5.05$ (s, $\left.2 \mathrm{H}, 4^{\prime \prime}{ }^{\prime} \mathrm{OCH}_{2} \mathrm{C}_{6} \mathrm{H}_{5}\right), 6.88$ (d, $J=$ $\left.8.7 \mathrm{~Hz}, 2 \mathrm{H}, 3^{\prime \prime}, 5^{\prime \prime}-\mathrm{H}\right), 7.13$ (d, $\left.J=8.7 \mathrm{~Hz}, 2 \mathrm{H}, 2^{\prime \prime}, 6^{\prime \prime}-\mathrm{H}\right), 7.18$ 7.27 (m, 5 H, 2', 3', $\left.4^{\prime}, 5^{\prime}, 6^{\prime}-\mathrm{H}\right), 7.33-7.45$ (m, $6 \mathrm{H}, 7-\mathrm{H}, 2,3,4,5,6-\mathrm{H}$ of $\left.4^{\prime \prime}-\mathrm{OCH}_{2} \mathrm{C}_{6} H_{5}\right), 7.52(\mathrm{~d}, J=7.9 \mathrm{~Hz}, 1 \mathrm{H}, 5-\mathrm{H}), 7.54(\mathrm{~s}, 1 \mathrm{H}, 4-$ H), $7.74(\mathrm{dt}, J=7.9,1.6 \mathrm{~Hz}, 1 \mathrm{H}, 6-\mathrm{H}), 8.35(\mathrm{~s}, 1 \mathrm{H}, 1-\mathrm{H}), 8.37$ $(\mathrm{dd}, J=7.9,1.6 \mathrm{~Hz}, 1 \mathrm{H}, 8-\mathrm{H}) \mathrm{ppm} .{ }^{13} \mathrm{C} \mathrm{NMR}: \delta=70.0\left(4^{\prime \prime}-\right.$ $\left.\mathrm{OCH}_{2} \mathrm{C}_{6} \mathrm{H}_{5}\right), 114.5\left(\mathrm{C}-3^{\prime \prime}, 5^{\prime \prime}\right), 118.0$ (C-5), 119.2 (C-4), $120.4(\mathrm{C}-$ 9a), 122.0 (C-8a), 123.9 (C-7), 126.76 (C-8), 126.84 (C-4'), 127.5 $\left(\mathrm{C}-2,6\right.$ of $\left.4^{\prime \prime}{ }^{-}-\mathrm{OCH}_{2} \mathrm{C}_{6} \mathrm{H}_{5}\right), 128.0\left(\mathrm{C}-4\right.$ of $\left.4^{\prime \prime}{ }^{\prime}-\mathrm{OCH}_{2} \mathrm{C}_{6} \mathrm{H}_{5}\right), 128.1(\mathrm{C}-$ $\left.2^{\prime}, 6^{\prime}\right), 128.4(\mathrm{C}-1), 128.6\left(\mathrm{C}-3,5\right.$ of $\left.4^{\prime \prime}-\mathrm{OCH}_{2} C_{6} \mathrm{H}_{5}\right), 129.9\left(\mathrm{C}-3^{\prime}, 5^{\prime}\right)$, $130.9\left(\mathrm{C}-2^{\prime \prime}, 6^{\prime \prime}\right), 132.4\left(\mathrm{C}-1^{\prime \prime}\right), 134.7$ (C-6), 136.7 (C-1 of 4' $\left.\mathrm{OCH}_{2} \mathrm{C}_{6} \mathrm{H}_{5}\right), 137.0\left(\mathrm{C}-1^{\prime}\right), 140.0(\mathrm{C}-2), 147.3(\mathrm{C}-3), 155.3(\mathrm{C}-4 \mathrm{a})$, 156.3 (C-4b), 158.4 (C-4" ), 177.0 (C-9) ppm. EI-MS: $\mathrm{m} / \mathrm{z}(\%)=$ $454\left[^{[M]^{+*}}\right.$ (33), 408 (2), 363 (8), 333 (2), 305 (3), 236 (3), 91 (100), 65 (3). $\mathrm{C}_{32} \mathrm{H}_{22} \mathrm{O}_{3}$ (454.52): calcd. for $\mathrm{C} 84.56, \mathrm{H} \mathrm{4.88}$; found $\mathrm{C}$ 84.29, H 4.87.

3-(4-Benzyloxyphenyl)-2-phenyl-3,4-dihydro-9H-xanthen-9-one (6d): Yellowish oil. ${ }^{1} \mathrm{H}$ NMR: $\delta=2.97\left(\mathrm{dd}, J=17.4,1.5 \mathrm{~Hz}, 1 \mathrm{H}, 4_{\text {trans }^{-}}\right.$ $\mathrm{H}), 3.63\left(\mathrm{dd}, J=17.4,8.4 \mathrm{~Hz}, 1 \mathrm{H}, 4_{\text {cis }}-\mathrm{H}\right), 4.26(\mathrm{brd}, J=8.4 \mathrm{~Hz}$, $1 \mathrm{H}, 3-\mathrm{H}), 4.95\left(\mathrm{~s}, 2 \mathrm{H}, 4^{\prime \prime}{ }^{\prime}-\mathrm{OCH}_{2} \mathrm{C}_{6} \mathrm{H}_{5}\right) 6.85(\mathrm{~d}, J=8.7 \mathrm{~Hz}, 2 \mathrm{H}$, $\left.3^{\prime \prime}, 5^{\prime \prime}-\mathrm{H}\right), 7.22\left(\mathrm{~d}, J=8.7 \mathrm{~Hz}, 2 \mathrm{H}, 2^{\prime \prime}, 6^{\prime \prime}-\mathrm{H}\right), 7.24-7.41(\mathrm{~m}, 10 \mathrm{H}$, $5,7,3^{\prime}, 4^{\prime}, 5^{\prime}-\mathrm{H}, 2,3,4,5,6-\mathrm{H}$ of $\left.4^{\prime \prime}{ }^{\prime}-\mathrm{OCH}_{2} \mathrm{C}_{6} H_{5}\right), 7.46$ (m, $2 \mathrm{H}, 2^{\prime}, 6^{\prime}-$ H), $7.54(\mathrm{~s}, 1 \mathrm{H}, 1-\mathrm{H}), 7.59$ (dt, $J=7.8,1.6 \mathrm{~Hz}, 1 \mathrm{H}, 6-\mathrm{H}), 8.28$ $(\mathrm{dd}, J=7.9,1.6 \mathrm{~Hz}, 1 \mathrm{H}, 8-\mathrm{H}) \mathrm{ppm} .{ }^{13} \mathrm{C} \mathrm{NMR}: \delta=36.8(\mathrm{C}-4)$, 40.9 (C-3), 69.9 (4'" $\left.-\mathrm{OC} \mathrm{H}_{2} \mathrm{C}_{6} \mathrm{H}_{5}\right), 115.1\left(\mathrm{C}-3^{\prime \prime}{ }^{\prime}, 5^{\prime \prime}\right), 116.5(\mathrm{C}-1)$, 116.8 (C-9a), 118.0 (C-5), 123.9 (C-8a), 125.0 (C-7), 125.6 (C-2', 6'), $126.2(\mathrm{C}-8), 127.49\left(\mathrm{C}-2,6\right.$ of $\left.4^{\prime \prime}{ }^{-}-\mathrm{OCH}_{2} \mathrm{C}_{6} \mathrm{H}_{5}\right), 127.52\left(\mathrm{C}-4^{\prime}\right), 127.9$ $\left(\mathrm{C}-4\right.$ of $\left.4^{\prime \prime}{ }^{\prime}-\mathrm{OCH}_{2} \mathrm{C}_{6} \mathrm{H}_{5}\right), 128.41,128.44$ and $128.5\left(\mathrm{C}-3^{\prime}, 5^{\prime}, \mathrm{C}-\right.$ $2^{\prime \prime}, 6^{\prime \prime}, \mathrm{C}-3,5$ of $\left.4^{\prime \prime}-\mathrm{OCH}_{2} \mathrm{C}_{6} \mathrm{H}_{5}\right), 132.8\left(\mathrm{C}-1^{\prime \prime}\right), 133.0(\mathrm{C}-6), 135.6$ $(\mathrm{C}-2), 136.8\left(\mathrm{C}-1\right.$ of $\left.4^{\prime \prime}{ }^{\prime}-\mathrm{OCH}_{2} \mathrm{C}_{6} \mathrm{H}_{5}\right), 139.1\left(\mathrm{C}-1^{\prime}\right), 155.8(\mathrm{C}-4 \mathrm{~b})$, 157.9 (C-4" ) 162.6 (C-4a), 174.2 (C-9) ppm. EI-MS: $m / z(\%)=456$ $[\mathrm{M}]^{+\cdot}(59), 455$ (11), 454 (9), 379 (4), 365 (20), 289 (5), 272 (13), 215 (5), 121 (4), 92 (11), 91 (100), 77 (3), 65 (8). HRMS (EI): calcd. for $\mathrm{C}_{32} \mathrm{H}_{24} \mathrm{O}_{3}[\mathrm{M}]^{+\cdot} 456.1725$; found 456.1725.

3-(4-Benzyloxyphenyl)-2-(4-methoxyphenyl)-9H-xanthen-9-one (5e): M.p. $140-142{ }^{\circ} \mathrm{C}$. ${ }^{1} \mathrm{H}$ NMR: $\delta=3.81\left(\mathrm{~s}, 3 \mathrm{H}, 4^{\prime}-\mathrm{OCH}_{3}\right), 5.06(\mathrm{~s}, 2$ $\left.\mathrm{H}, 4^{\prime \prime}-\mathrm{OCH}_{2} \mathrm{C}_{6} \mathrm{H}_{5}\right), 6.80$ (d, $\left.J=8.8 \mathrm{~Hz}, 2 \mathrm{H}, 3^{\prime}, 5^{\prime}-\mathrm{H}\right), 6.90$ (d, $J$ $\left.=8.8 \mathrm{~Hz}, 2 \mathrm{H}, 3^{\prime \prime}, 5^{\prime \prime}-\mathrm{H}\right), 7.11\left(\mathrm{~d}, J=8.8 \mathrm{~Hz}, 2 \mathrm{H}, 2^{\prime}, 6^{\prime}-\mathrm{H}\right), 7.15$ $\left(\mathrm{d}, J=8.8 \mathrm{~Hz}, 2 \mathrm{H}, 2^{\prime \prime}, 6^{\prime \prime}-\mathrm{H}\right), 7.34-7.46\left(\mathrm{~m}, 6 \mathrm{H}, 7-\mathrm{H}, 4^{\prime \prime}\right.$ $\left.\mathrm{OCH}_{2} \mathrm{C}_{6} H_{5}\right), 7.520(\mathrm{~d}, J=7.8 \mathrm{~Hz}, 1 \mathrm{H}, 5-\mathrm{H}), 7.522(\mathrm{~s}, 1 \mathrm{H}, 4-\mathrm{H})$, $7.74(\mathrm{dt}, J=7.8,1.6 \mathrm{~Hz}, 1 \mathrm{H}, 6-\mathrm{H}), 8.32$ (s, $1 \mathrm{H}, 1-\mathrm{H}), 8.37$ (dd, $J$ $=8.0,1.6 \mathrm{~Hz}, 1 \mathrm{H}, 8-\mathrm{H}) \mathrm{ppm} .{ }^{13} \mathrm{C} \mathrm{NMR}: \delta=55.2\left(4^{\prime}-\mathrm{OCH}_{3}\right), 70.0$ $\left(4^{\prime \prime}-\mathrm{OCH}_{2} \mathrm{C}_{6} \mathrm{H}_{5}\right), 113.6\left(\mathrm{C}-3^{\prime}, 5^{\prime}\right), 114.5\left(\mathrm{C}-3^{\prime \prime}, 5^{\prime \prime}\right), 118.0(\mathrm{C}-5)$, 119.2 (C-4), 120.4 (C-9a), 122.0 (C-8a), 123.9 (C-7), 126.8 (C-8), $127.6\left(\mathrm{C}-2,6\right.$ of $\left.4^{\prime \prime}{ }^{-}-\mathrm{OCH}_{2} \mathrm{C}_{6} \mathrm{H}_{5}\right), 128.1\left(\mathrm{C}-4\right.$ of $\left.4{ }^{\prime \prime}{ }^{-}-\mathrm{OCH}_{2} \mathrm{C}_{6} \mathrm{H}_{5}\right)$, $128.2(\mathrm{C}-1), 128.6\left(\mathrm{C}-3,5\right.$ of $\left.4^{\prime \prime}-\mathrm{OCH}_{2} \mathrm{C}_{6} \mathrm{H}_{5}\right), 130.9\left(\mathrm{C}-2^{\prime \prime}, 6^{\prime \prime}\right)$, $131.0\left(\mathrm{C}-2^{\prime}, 6^{\prime}\right), 132.4\left(\mathrm{C}-1^{\prime}\right), 132.6\left(\mathrm{C}-1^{\prime \prime}\right), 134.7(\mathrm{C}-6), 136.65(\mathrm{C}-$ 2), $136.72\left(\mathrm{C}-1\right.$ of $\left.4^{\prime \prime}{ }^{-} \mathrm{OCH}_{2} \mathrm{C}_{6} \mathrm{H}_{5}\right), 147.3(\mathrm{C}-3), 155.1$ (C-4a), 156.3 (C-4b), 158.3 (C-4' '), 158.6 (C-4'), 177.1 (C-9) ppm. EI-MS: $m / z$ $(\%)=484[\mathrm{M}]^{+\cdot}(61), 394(15), 393(23), 91(100), 85(6) . \mathrm{C}_{33} \mathrm{H}_{24} \mathrm{O}_{4}$ (484.54): calcd. for C 81.80, H 4.99; found C 82.02, H 5.06.

3-(4-Benzyloxyphenyl)-2-(4-methoxyphenyl)-3,4-dihydro-9Hxanthen-9-one (6e): Yellowish oil. ${ }^{1} \mathrm{H}$ NMR: $\delta=2.95$ (dd, $J=17.3$, $\left.1.4 \mathrm{~Hz}, 1 \mathrm{H}, 4_{\text {trans }}-\mathrm{H}\right), 3.60\left(\mathrm{dd}, J=17.3,8.4 \mathrm{~Hz}, 1 \mathrm{H}, 4_{\text {cis }}-\mathrm{H}\right), 3.77$ (s, $3 \mathrm{H}, 4^{\prime}-\mathrm{OCH}_{3}$ ), 4.22 (brd, $\left.J=8.4 \mathrm{~Hz}, 1 \mathrm{H}, 3-\mathrm{H}\right), 4.94$ (s, $2 \mathrm{H}$, $\left.4^{\prime \prime}-\mathrm{OCH}_{2} \mathrm{C}_{6} \mathrm{H}_{5}\right), 6.81\left(\mathrm{~d}, J=8.9 \mathrm{~Hz}, 1 \mathrm{H}, 3^{\prime}, 5^{\prime}-\mathrm{H}\right), 6.84$ (d, $J=$ $\left.8.7 \mathrm{~Hz}, 2 \mathrm{H}, 3^{\prime \prime}, 5^{\prime \prime}-\mathrm{H}\right), 7.20$ (d, $\left.J=8.7 \mathrm{~Hz}, 2 \mathrm{H}, 2^{\prime \prime}, 6^{\prime \prime}-\mathrm{H}\right), 7.28$ $7.39\left(\mathrm{~m}, 7 \mathrm{H}, 5-\mathrm{H}, 7-\mathrm{H}, 2,3,4,5,6-\mathrm{H}\right.$ of $\left.4^{\prime \prime}{ }^{\prime}-\mathrm{OCH}_{2} \mathrm{C}_{6} H_{5}\right), 7.43$ (s, 1 $\mathrm{H}, 1-\mathrm{H}), 7.43\left(\mathrm{~d}, J=8.9 \mathrm{~Hz}, 2 \mathrm{H}, 2^{\prime}, 6^{\prime}-\mathrm{H}\right), 7.58(\mathrm{dt}, J=7.8,1.6 \mathrm{~Hz}$, $1 \mathrm{H}, 6-\mathrm{H}), 8.27$ (dd, $J=8.0,1.6 \mathrm{~Hz}, 1 \mathrm{H}, 8-\mathrm{H}) \mathrm{ppm} .{ }^{13} \mathrm{C} \mathrm{NMR}: \delta$ $=36.7(\mathrm{C}-4), 40.8(\mathrm{C}-3), 55.2\left(4^{\prime}-\mathrm{OCH}_{3}\right), 69.8\left(4^{\prime \prime}{ }^{\prime}-\mathrm{OCH}_{2} \mathrm{C}_{6} \mathrm{H}_{5}\right)$, $113.8\left(\mathrm{C}-3^{\prime}, 5^{\prime}\right), 114.5$ (C-1), $115.0\left(\mathrm{C}-3^{\prime \prime}, 5^{\prime \prime}\right), 116.9$ (C-9a), 117.9 (C-5), 123.8 (C-8a), 124.9 (C-7), 126.1 (C-8), 126.8 (C-2',6'), 127.4 $\left(\mathrm{C}-2,6\right.$ of $\left.4^{\prime \prime}{ }^{\prime}-\mathrm{OCH}_{2} \mathrm{C}_{6} \mathrm{H}_{5}\right), 127.9\left(\mathrm{C}-4\right.$ of $\left.4^{\prime \prime}{ }^{\prime}-\mathrm{OCH}_{2} \mathrm{C}_{6} \mathrm{H}_{5}\right), 128.3(\mathrm{C}-$ $\left.2^{\prime \prime}, 6^{\prime \prime}\right), 128.5\left(\mathrm{C}-3,5\right.$ of $\left.4^{\prime \prime}-\mathrm{OCH}_{2} \mathrm{C}_{6} \mathrm{H}_{5}\right), 131.5\left(\mathrm{C}-1^{\prime}\right), 132.87(\mathrm{C}-$ 6), $132.90\left(\mathrm{C}-1^{\prime \prime}\right), 135.2(\mathrm{C}-2), 136.8\left(\mathrm{C}-1\right.$ of $\left.4^{\prime \prime}{ }^{\prime}-\mathrm{OCH}_{2} \mathrm{C}_{6} \mathrm{H}_{5}\right), 155.8$ (C-4b), 157.9 (C-4"'), 155.9 (C-4'), 162.1 (C-4a), 174.2 (C-9) ppm. EI-MS: $m / z(\%)=486[\mathrm{M}]^{+*}(44), 485(7), 484(9), 408(6), 395(15)$, 364 (3), 302 (5), 213 (3), 135 (33), 121 (8), 92 (14), 91 (100), 77 (7), 65 (9). HRMS (EI): calcd. for $\mathrm{C}_{33} \mathrm{H}_{26} \mathrm{O}_{4}[\mathrm{M}]^{+\cdot} 486.1831$; found 486.1829 .

3-(4-Benzyloxyphenyl)-2-(3,4-dimethoxyphenyl)-9H-xanthen-9-one (5f): M.p. ${ }^{188-189}{ }^{\circ} \mathrm{C} .{ }^{1} \mathrm{H}$ NMR: $\delta=3.61$ (s, $3 \mathrm{H}, 4^{\prime}-\mathrm{OCH}_{3}$ ), 3.89 $\left(\mathrm{s}, 3 \mathrm{H}, 3^{\prime}-\mathrm{OCH}_{3}\right), 5.06\left(\mathrm{~s}, 2 \mathrm{H}, 4^{\prime \prime}-\mathrm{OCH}_{2} \mathrm{C}_{6} \mathrm{H}_{5}\right), 6.59(\mathrm{~d}, J=$ $\left.1.8 \mathrm{~Hz}, 1 \mathrm{H}, 2^{\prime}-\mathrm{H}\right), 6.79-6.86\left(\mathrm{~m}, 2 \mathrm{H}, 5^{\prime}-\mathrm{H}, 6^{\prime}-\mathrm{H}\right), 6.90$ (d, $J=$ $\left.8.8 \mathrm{~Hz}, 2 \mathrm{H}, 3^{\prime \prime}, 5^{\prime \prime}-\mathrm{H}\right), 7.15$ (d, $\left.J=8.8 \mathrm{~Hz}, 2 \mathrm{H}, 2^{\prime \prime}, 6^{\prime \prime}-\mathrm{H}\right), 7.31-$ $7.46\left(\mathrm{~m}, 6 \mathrm{H}, 7-\mathrm{H}, 4^{\prime \prime}{ }^{-}-\mathrm{OCH}_{2} \mathrm{C}_{6} H_{5}\right), 7.52(\mathrm{~d}, J=7.9 \mathrm{~Hz}, 1 \mathrm{H}, 5-\mathrm{H})$, $7.53(\mathrm{~s}, 1 \mathrm{H}, 4-\mathrm{H}), 7.75(\mathrm{dt}, J=7.9,1.8 \mathrm{~Hz}, 1 \mathrm{H}, 6-\mathrm{H}), 8.36(\mathrm{~s}, 1$ $\mathrm{H}, 1-\mathrm{H}), 8.38$ (dd, $J=8.2,1.8 \mathrm{~Hz}, 1 \mathrm{H}, 8-\mathrm{H}) \mathrm{ppm} .{ }^{13} \mathrm{C} \mathrm{NMR}: \delta=$ $55.6\left(4^{\prime}-\mathrm{OCH}_{3}\right), 55.8\left(3^{\prime}-\mathrm{OCH}_{3}\right), 69.9\left(4^{\prime \prime}-\mathrm{OCH}_{2} \mathrm{C}_{6} \mathrm{H}_{5}\right), 110.8(\mathrm{C}-$ $\left.5^{\prime}\right), 113.4\left(\mathrm{C}-2^{\prime}\right), 114.6\left(\mathrm{C}-3^{\prime \prime}, 5^{\prime \prime}\right), 118.0$ (C-5), 119.2 (C-4), 120.4 (C-9a), 121.9 (C-8a), 122.2 (C-6'), 123.9 (C-7), 126.8 (C-8), 127.4 $\left(\mathrm{C}-2,6\right.$ of $\left.4^{\prime \prime}{ }^{-} \mathrm{OCH}_{2} \mathrm{C}_{6} \mathrm{H}_{5}\right), 127.9$ (C-1), 128.0 (C-4 of 4' ' $\left.\mathrm{OCH}_{2} \mathrm{C}_{6} \mathrm{H}_{5}\right), 128.6\left(\mathrm{C}-3,5\right.$ of $\left.4^{\prime \prime}-\mathrm{OCH}_{2} \mathrm{C}_{6} \mathrm{H}_{5}\right), 130.8\left(\mathrm{C}-2^{\prime \prime}{ }^{\prime}, 6^{\prime \prime}\right)$, $132.5\left(\mathrm{C}-1^{\prime}\right), 132.7\left(\mathrm{C}-1^{\prime \prime}\right), 134.7$ (C-6), 136.7 (C-2, C-1 of $4^{\prime \prime}$ $\left.\mathrm{OCH}_{2} \mathrm{C}_{6} \mathrm{H}_{5}\right), 147.3(\mathrm{C}-3), 148.0\left(\mathrm{C}-3^{\prime}\right), 148.2\left(\mathrm{C}-4^{\prime}\right), 155.1$ (C-4a), 156.3 (C-4b), 158.3 (C-4" ), 177.0 (C-9) ppm. EI-MS: m/z (\%) = $514\left[\mathrm{M}^{+*}(11), 388\right.$ (11), 166 (11), 165 (13), 91 (100), 77 (3), 65 (5). HRMS (EI): calcd. for $\mathrm{C}_{34} \mathrm{H}_{26} \mathrm{O}_{5}[\mathrm{M}]^{+\cdot} 514.1780$; found 514.1785 .

Synthesis of the $(E)$-2-Methyl-3-styryl-4H-chromen-4-ones 7a-c: The appropriate styrene $(\mathbf{4 a}-\mathbf{c}, 2.5 \mathrm{mmol})$ was added to a mixture of 3-bromo-2-methyl-4H-chromen-4-one (1, $0.12 \mathrm{~g}, 0.5 \mathrm{mmol})$, triphenylphosphane (13.1 mg, $0.05 \mathrm{mmol})$, palladium chloride (4.4 mg, $0.025 \mathrm{mmol})$ and triethylamine $(69.7 \mu \mathrm{L}, 0.5 \mathrm{mmol})$ in $N$ methyl-2-pyrrolidin-1-one $(5 \mathrm{~mL})$. The mixture was stirred at $160{ }^{\circ} \mathrm{C}$ for $9 \mathrm{~h}$. It was then poured into water $(15 \mathrm{~mL})$ and ice $(10 \mathrm{~g})$, extracted with diethyl ether $(4 \times 25 \mathrm{~mL})$ and dried with anhydrous sodium sulfate. The residue was concentrated, taken up in dichloromethane $(10 \mathrm{~mL})$ and purified by thin layer chromatography (eluent: dichloromethane/light petroleum 7:3). The obtained (E)-2-methyl-3-styryl-4H-chromen-4-ones (7a-c) were recrystallised from ethanol and obtained in moderate to good yields.

(E)-2-Methyl-3-(2-phenylvinyl)-4H-chromen-4-one (7a): Yield $6.30 \mathrm{mg}(48 \%)$; m.p. $136-137^{\circ} \mathrm{C} .{ }^{1} \mathrm{H}$ NMR: $\delta=2.62$ (s, $3 \mathrm{H}, 2-$ $\left.\mathrm{CH}_{3}\right), 6.97(\mathrm{~d}, J=16.3 \mathrm{~Hz}, 1 \mathrm{H}, \alpha-\mathrm{H}), 7.24-7.29\left(\mathrm{~m}, 1 \mathrm{H}, 4^{\prime}-\mathrm{H}\right)$, $7.36\left(\mathrm{~d}, J=7.7 \mathrm{~Hz}, 2 \mathrm{H}, 3^{\prime}, 5^{\prime}-\mathrm{H}\right), 7.38$ (ddd, $J=8.0,7.9,1.0 \mathrm{~Hz}$, $2 \mathrm{H}, 6-\mathrm{H}), 7.41(\mathrm{~d}, J=8.2 \mathrm{~Hz}, 1 \mathrm{H}, 8-\mathrm{H}), 7.53$ (dd, $J=7.7,1.5 \mathrm{~Hz}$, $\left.2 \mathrm{H}, 2^{\prime}, 6^{\prime}-\mathrm{H}\right), 7.63$ (ddd, $\left.J=8.2,8.0,1.6 \mathrm{~Hz}, 1 \mathrm{H}, 7-\mathrm{H}\right), 7.71$ (d, $J$ $=16.3 \mathrm{~Hz}, 1 \mathrm{H}, \beta-\mathrm{H}), 8.25(\mathrm{dd}, J=7.9,1.6 \mathrm{~Hz}, 1 \mathrm{H}, 5-\mathrm{H}) \mathrm{ppm}$. ${ }^{13} \mathrm{C}$ NMR: $\delta=19.3\left(2-\mathrm{CH}_{3}\right), 117.5(\mathrm{C}-8), 118.5(\mathrm{C}-3), 119.4(\mathrm{C}-\alpha)$, 123.4 (C-10), 124.8 (C-6), 126.1 (C-5), 126.4 (C-2',6'), 127.7 (C-4'), $128.6\left(\mathrm{C}^{-} 3^{\prime}, 5^{\prime}\right), 133.1$ (C-7), 134.2 (C- $\left.\beta\right), 137.9$ (C-1'), 155.2 (C-9), 163.8 (C-2), 176.9 (C-4) ppm. EI-MS: $m / z(\%)=262[\mathrm{M}]^{+\cdot}(100)$, 247 (39), 233 (7), 218 (5), 202 (3), 191 (5), 185 (63), 141 (27), 121 (14), 115 (21), 102 (3), 92 (11), 77 (8), 63 (10), 51 (6). $\mathrm{C}_{18} \mathrm{H}_{14} \mathrm{O}_{2}$ (262.30): calcd. for C 82.42, H 5.38; found C 82.31, H 5.46. 
(E)-3-[2-(4-Methoxyphenyl)vinyl]-2-methyl-4H-chromen-4-one (7b): Yield $71.6 \mathrm{mg}$ (49\%); m.p. 89-91 ${ }^{\circ} \mathrm{C} .{ }^{1} \mathrm{H}$ NMR: $\delta=2.61$ (s, $3 \mathrm{H}$, 2-C $\left.\mathrm{CH}_{3}\right), 3.83\left(\mathrm{~s}, 3 \mathrm{H}, 4^{\prime}-\mathrm{OCH}_{3}\right), 6.83(\mathrm{~d}, J=16.3 \mathrm{~Hz}, 1 \mathrm{H}, \alpha-\mathrm{H})$, $6.90\left(\mathrm{~d}, J=8.7 \mathrm{~Hz}, 2 \mathrm{H}, 3^{\prime}, 5^{\prime}-\mathrm{H}\right), 7.35-7.43(\mathrm{~m}, 2 \mathrm{H}, 6-\mathrm{H}, 8-\mathrm{H})$, $7.47\left(\mathrm{~d}, J=8.7 \mathrm{~Hz}, 2 \mathrm{H}, 2^{\prime}, 6^{\prime}-\mathrm{H}\right), 7.60-7.67$ (m, $\left.1 \mathrm{H}, 7-\mathrm{H}\right), 7.64$ $(\mathrm{d}, J=16.3 \mathrm{~Hz}, 1 \mathrm{H}, \beta-\mathrm{H}), 8.25(\mathrm{dd}, J=8.1,0.9 \mathrm{~Hz}, 1 \mathrm{H}, 5-$ H) ppm. ${ }^{13} \mathrm{C}$ NMR: $\delta=19.3\left(2-\mathrm{CH}_{3}\right), 55.3\left(4^{\prime}-\mathrm{OCH}_{3}\right), 114.0(\mathrm{C}-$ $\left.3^{\prime}, 5^{\prime}\right), 117.3$ (C-8), 117.5 (C- $\alpha$ ), 118.7 (C-3), 123.4 (C-10), 124.8 (C6), 126.1 (C-5), $127.7\left(\mathrm{C}-2^{\prime}, 6^{\prime}\right), 130.7\left(\mathrm{C}-1^{\prime}\right), 133.1$ (C-7), 133.8 (Cß), 155.3 (C-9), 159.3 (C-4'), 163.3 (C-2), 177.0 (C-4) ppm. EI-MS: $m / z(\%)=292[\mathrm{M}]^{+\cdot}(31), 277(14), 211(8), 197(10), 183(11), 169$ (13), 155 (17), 141 (19), 127 (24), 125 (10), 113 (28), 111 (16), 99 (34), 97 (27), 85 (96), 83 (20), 71 (100), 69 (18). HRMS (EI): calcd. for $\mathrm{C}_{19} \mathrm{H}_{16} \mathrm{O}_{3}[\mathrm{M}]^{+\cdot} 292.1099$; found 292.1091.

(E)-3-[2-(3,4-Dimethoxyphenyl)vinyl]-2-methyl-4H-chromen-4-one (7c): Yield $83.8 \mathrm{mg}(52 \%)$; m.p. $117-118^{\circ} \mathrm{C} .{ }^{1} \mathrm{H}$ NMR: $\delta=2.61(\mathrm{~s}$, $\left.3 \mathrm{H}, 2-\mathrm{CH}_{3}\right), 3.90$ and $3.93\left(2 \times \mathrm{s}, 6 \mathrm{H}, 3^{\prime}, 4^{\prime}-\mathrm{OCH}_{3}\right), 6.77-6.92(\mathrm{~m}$, $\left.1 \mathrm{H}, 5^{\prime}-\mathrm{H}\right), 6.84(\mathrm{~d}, J=16.3 \mathrm{~Hz}, 1 \mathrm{H}, \alpha-\mathrm{H}), 7.06-7.07(\mathrm{~m}, 2 \mathrm{H}$, $\left.2^{\prime}, 6^{\prime}-\mathrm{H}\right), 7.35-7.41(\mathrm{~m}, 2 \mathrm{H}, 6-\mathrm{H}, 8-\mathrm{H}), 7.59$ (d, $J=16.3 \mathrm{~Hz}, 1 \mathrm{H}$, $\beta-\mathrm{H}), 7.59-7.65(\mathrm{~m}, 1 \mathrm{H}, 7-\mathrm{H}), 8.24(\mathrm{dd}, J=7.7,1.2 \mathrm{~Hz}, 1 \mathrm{H}, 5-$ H) ppm. ${ }^{13} \mathrm{C}$ NMR: $\delta=19.4\left(2-\mathrm{CH}_{3}\right), 55.8$ and $55.9\left(3^{\prime}, 4^{\prime}-\mathrm{OCH}_{3}\right)$, $109.0\left(\mathrm{C}-2^{\prime}\right), 111.0\left(\mathrm{C}-5^{\prime}\right), 117.5(\mathrm{C}-8, \mathrm{C}-\alpha), 118.6(\mathrm{C}-3), 119.4(\mathrm{C}-$ 6'), 123.2 (C-10), 124.7 (C-6), 126.0 (C-5), 130.9 (C-1'), 133.1 (C7), $134.0(\mathrm{C}-\beta), 148.8$ and $148.9\left(\mathrm{C}-3^{\prime}, 4^{\prime}\right), 155.2$ (C-9), 163.3 (C-2), 176.9 (C-4) ppm. EI-MS: $m / z(\%)=322[\mathrm{M}]^{+*}(100), 307(30), 292$ (6), 276 (6), 263 (6), 247 (7), 235 (9), 221 (3), 202 (3), 185 (17), 161 (9), 144 (5), 138 (7), 121 (10), 115 (15), 92 (13), 77 (7), 63 (9), 51 (6). $\mathrm{C}_{20} \mathrm{H}_{18} \mathrm{O}_{4}$ (322.35): calcd. for $\mathrm{C} 74.52$, H 5.63; found $\mathrm{C} 74.36$, H 5.86 .

Synthesis of the $(E, E)-2,3-D i s t y r y l-4 H$-chromen-4-ones 8a-c: Sodium $(0.4 \mathrm{~g}, 16.7 \mathrm{mmol})$ was gradually added to dry methanol $(20 \mathrm{~mL})$ and the mixture was stirred until the reaction mixture had reached room temperature. The appropriate (E)-2-methyl-3-styryl$4 H$-chromen-4-one (7a-c, $4.2 \mathrm{mmol})$ and benzaldehyde (2a, $0.53 \mathrm{~g}$, $5.0 \mathrm{mmol}$ ) were added and the reaction mixture was allowed to stand at room temperature for $48 \mathrm{~h}$. After this period, the solution was poured into ice $(30 \mathrm{~g})$ and water $(50 \mathrm{~mL})$ and the $\mathrm{pH}$ was adjusted to 4 with hydrochloric acid. The yellow solid was removed by filtration, taken up in dichloromethane $(10 \mathrm{~mL})$ and purified by thin layer chromatography with dichloromethane as eluent. The solvent was evaporated to dryness and the residue were recrystallised from ethanol to give the $(E, E)$-2,3-distyryl-4H-chromen-4ones $\mathbf{8 a}-\mathbf{c}$.

$(\boldsymbol{E}, \boldsymbol{E})$-2,3-Bis(2-phenylvinyl)-4H-chromen-4-one (8a): Yield $0.78 \mathrm{~g}$ (53\%); m.p. $160-162^{\circ} \mathrm{C} .{ }^{1} \mathrm{H}$ NMR: $\delta=7.21(\mathrm{~d}, J=15.8 \mathrm{~Hz}, 1 \mathrm{H}$, $\left.\alpha^{\prime}-\mathrm{H}\right), 7.29\left(\mathrm{tt}, J=7.2,1.7 \mathrm{~Hz}, 1 \mathrm{H}, 4^{\prime}-\mathrm{H}\right), 7.33-7.43(\mathrm{~m}, 6 \mathrm{H}, 6-$ $\left.\mathrm{H}, 3^{\prime}, 5^{\prime}, 3^{\prime \prime}, 4^{\prime \prime}, 5^{\prime \prime}-\mathrm{H}\right), 7.37(\mathrm{~d}, J=15.7 \mathrm{~Hz}, 1 \mathrm{H}, \alpha-\mathrm{H}), 7.43(\mathrm{~d}, J$ $\left.=15.8 \mathrm{~Hz}, 1 \mathrm{H}, \beta^{\prime}-\mathrm{H}\right), 7.50(\mathrm{~d}, J=8.1 \mathrm{~Hz}, 1 \mathrm{H}, 8-\mathrm{H}), 7.54-7.59$ (m, $\left.4 \mathrm{H}, 2^{\prime}, 6^{\prime}, 2^{\prime \prime}, 6^{\prime \prime}-\mathrm{H}\right), 7.65(\mathrm{dt}, J=8.1,1.7 \mathrm{~Hz}, 1 \mathrm{H}, 7-\mathrm{H}), 7.67$ $(\mathrm{d}, J=15.7 \mathrm{~Hz}, 1 \mathrm{H}, \beta-\mathrm{H}), 8.22(\mathrm{dd}, J=7.9,1.7 \mathrm{~Hz}, 1 \mathrm{H}, 5-$ H) ppm. ${ }^{13} \mathrm{C}$ NMR: $\delta=117.5$ (C-8), 118.5 (C- $\left.\alpha\right), 119.0$ (C-3), 119.1 $\left(\mathrm{C}-\alpha^{\prime}\right), 123.1$ (C-10), 124.7 (C-6), $126.0(\mathrm{C}-5), 126.7$ (C-2', 6') 127.7 $\left(\mathrm{C}-2^{\prime \prime}, 6^{\prime \prime}\right), 127.9\left(\mathrm{C}-4^{\prime}\right), 128.6\left(\mathrm{C}-3^{\prime}, 5^{\prime}\right), 129.0\left(\mathrm{C}-3^{\prime \prime}, 5^{\prime \prime}\right), 129.7(\mathrm{C}-$ $\left.4^{\prime \prime}\right), 133.5$ (C-7), $135.5\left(\mathrm{C}-1^{\prime \prime}\right), 136.1\left(\mathrm{C}-\beta^{\prime}\right), 137.1$ (C- $\left.\beta\right), 137.5$ (C1'), 154.9 (C-9), 158.0 (C-2), 177.5 (C-4) ppm. EI-MS: $m / z(\%)=$ $350 \mathrm{M}^{+\cdot}(58), 273$ (100), 259 (4), 228 (9), 215 (7), 202 (8), 152 (6), 121 (8), 115 (11), 92 (8), 77 (13), 63 (7), 51 (7). $\mathrm{C}_{25} \mathrm{H}_{18} \mathrm{O}_{2}$ (350.41): calcd. for C 85.69, H 5.18; found C 85.44, H 5.34.

2,3-Diphenyl-1,2-dihydro-9H-xanthen-9-one (9a): Yellowish oil. ${ }^{1} \mathrm{H}$ NMR: $\delta=3.19\left(\mathrm{dd}, J=16.8,8.4 \mathrm{~Hz}, 1 \mathrm{H}, 1_{c i s}-\mathrm{H}\right), 3.45(\mathrm{dd}, J=$ 16.8, $2.0 \mathrm{~Hz}, 1 \mathrm{H}, 1_{\text {trans }}-\mathrm{H}$ ), 4.33 (brd, $\left.J=8.4 \mathrm{~Hz}, 1 \mathrm{H}, 2-\mathrm{H}\right), 7.02$ (s, $1 \mathrm{H}, 4-\mathrm{H}), 7.15-7.23$ (m, $\left.3 \mathrm{H}, 3^{\prime}, 4^{\prime}, 5^{\prime}-\mathrm{H}\right), 7.25-7.27$ (m, $2 \mathrm{H}$, $\left.2^{\prime}, 6^{\prime}-\mathrm{H}\right), 7.32-7.41\left(\mathrm{~m}, 4 \mathrm{H}, 7,3^{\prime \prime}, 4^{\prime \prime}, 5^{\prime \prime}-\mathrm{H}\right), 7.48$ (d, $J=8.4 \mathrm{~Hz}, 1$ $\mathrm{H}, 5-\mathrm{H}), 7.50-7.53\left(\mathrm{~m}, 2 \mathrm{H}, 2^{\prime \prime}, 6^{\prime \prime}-\mathrm{H}\right), 7.60-7.66(\mathrm{~m}, 1 \mathrm{H}, 6-\mathrm{H})$, $8.16(\mathrm{dd}, J=8.1,1.1 \mathrm{~Hz}, 1 \mathrm{H}, 8-\mathrm{H}) \mathrm{ppm} .{ }^{13} \mathrm{C}$ NMR: $\delta=28.2(\mathrm{C}-$ 1), 41.5 (C-2), 111.5 (C-9a), 118.4 (C-4), 124.1 (C-8a), 124.7 (C-7), $125.7(\mathrm{C}-8), 126.8\left(\mathrm{C}-2^{\prime \prime}, 6^{\prime \prime}\right), 127.0\left(\mathrm{C}-4^{\prime}\right), 127.2\left(\mathrm{C}-2^{\prime}, 6^{\prime}\right), 128.8$ (C-3', 5', C-3" , 5' '), 129.3 (C-4' $), 133.0$ (C-6), 138.1 (C-1"'), 140.7 (C-1'), 150.6 (C-3), 155.6 (C-4a), 159.6 (C-4b), 176.7 (C-9) ppm. EI-MS: $m / z(\%)=350[\mathrm{M}]^{+\cdot}(84), 349(52), 274(55), 273(100), 261$ (14), 228 (10), 215 (12), 202 (8), 189 (6), 152 (10), 139 (7), 121 (12), 115 (11), 105 (33), 92 (8), 91 (6), 77 (23), 63 (7), 51 (12). HRMS (EI): calcd. for $\mathrm{C}_{25} \mathrm{H}_{18} \mathrm{O}_{2}[\mathrm{M}]^{+\cdot} 350.1307$; found 350.1306 .

$(E, E)-2-[2-(4-M e t h o x y p h e n y l) v i n y l]-3-(2-p h e n y l v i n y l)-4 H$-chromen4-one (8b): Yield $1.07 \mathrm{~g}(67 \%)$; m.p. $163-164{ }^{\circ} \mathrm{C} .{ }^{1} \mathrm{H}$ NMR: $\delta=$ $3.85\left(\mathrm{~s}, 3 \mathrm{H}, 4^{\prime \prime}-\mathrm{OCH}_{3}\right), 6.93\left(\mathrm{~d}, J=8.8 \mathrm{~Hz}, 2 \mathrm{H}, 3^{\prime \prime}, 5^{\prime \prime}-\mathrm{H}\right), 7.11$ $\left(\mathrm{d}, J=16.4 \mathrm{~Hz}, 1 \mathrm{H}, \alpha^{\prime}-\mathrm{H}\right), 7.36\left(\mathrm{~d}, J=16.4 \mathrm{~Hz}, 1 \mathrm{H}, \beta^{\prime}-\mathrm{H}\right), 7.36-$ $7.42\left(\mathrm{~m}, 4 \mathrm{H}, 6-\mathrm{H}, 3^{\prime}, 4^{\prime}, 5^{\prime}-\mathrm{H}\right), 7.42(\mathrm{~d}, J=16.0 \mathrm{~Hz}, 1 \mathrm{H}, \alpha-\mathrm{H})$, $7.52\left(\mathrm{~d}, J=8.8 \mathrm{~Hz}, 2 \mathrm{H}, 2^{\prime \prime}, 6^{\prime \prime}-\mathrm{H}\right), 7.51-7.52(\mathrm{~m}, 1 \mathrm{H}, 8-\mathrm{H}), 7.60$ $\left(\mathrm{dd}, J=7.9,1.7 \mathrm{~Hz}, 2 \mathrm{H}, 2^{\prime}, 6^{\prime}-\mathrm{H}\right), 7.68(\mathrm{dt}, J=7.8,1.6 \mathrm{~Hz}, 1 \mathrm{H}$, $7-\mathrm{H}), 7.70(\mathrm{~d}, J=16.0 \mathrm{~Hz}, 1 \mathrm{H}, \beta-\mathrm{H}), 8.25(\mathrm{dd}, J=8.0,1.6 \mathrm{~Hz}, 1$ $\mathrm{H}, 5-\mathrm{H}) \mathrm{ppm} .{ }^{13} \mathrm{C}$ NMR: $\delta=55.3\left(4^{\prime \prime}-\mathrm{OCH}_{3}\right), 114.1\left(5^{\prime \prime}, \mathrm{C}-3^{\prime \prime}\right)$, $116.9\left(\mathrm{C}-\alpha^{\prime}\right), 117.5(\mathrm{C}-8), 118.8(\mathrm{C}-\alpha), 119.5$ (C-3), 123.1 (C-10), 124.7 (C-6), 126.1 (C-5), $127.7\left(\mathrm{C}-2^{\prime}, 6^{\prime}\right), 128.0\left(\mathrm{C}-2^{\prime \prime}, 6^{\prime \prime}\right), 129.0(\mathrm{C}-$ $\left.3^{\prime}, 5^{\prime}\right), 129.4\left(\mathrm{C}-4^{\prime}\right), 130.4\left(\mathrm{C}-1^{\prime \prime}\right), 133.5(\mathrm{C}-7), 135.6\left(\mathrm{C}-1^{\prime}\right), 135.8$ $\left(\mathrm{C}-\beta^{\prime}\right), 136.8$ (C- $\beta$ ), 155.1 (C-9), 157.7 (C-2), 159.6 (C-4' $\left.{ }^{\prime \prime}\right), 177.7$ (C-4) ppm. EI-MS: $m / z(\%)=380\left[\mathrm{M}^{+\cdot}(100), 303(65), 289\right.$ (6), 273 (41), 260 (11), 215 (13), 202 (7), 190 (6), 151 (7), 121 (48), 115 (7), 92 (9), 77 (9), 63 (6), 51 (4). $\mathrm{C}_{26} \mathrm{H}_{20} \mathrm{O}_{3}$ (380.44): calcd. for $\mathrm{C}$ 82.08, H 5.30; found C 82.05, H 4.99 .

(E,E)-2-[2-(3,4-Dimethoxyphenyl)vinyl]-3-(2-phenylvinyl)-4Hchromen-4-one (8c): Yield $1.19 \mathrm{~g}(69 \%)$; m.p. $128-130{ }^{\circ} \mathrm{C} .{ }^{1} \mathrm{H}$ NMR: $\delta=3.93$ and 3.96 (s, $\left.6 \mathrm{H}, 3^{\prime \prime}, 4^{\prime \prime}-\mathrm{OCH}_{3}\right), 6.89(\mathrm{~d}, J=8.2 \mathrm{~Hz}$, $\left.1 \mathrm{H}, 5^{\prime \prime}-\mathrm{H}\right), 7.11\left(\mathrm{dd}, J=8.2,1.9 \mathrm{~Hz}, 1 \mathrm{H}, 6^{\prime \prime}-\mathrm{H}\right), 7.13(\mathrm{~d}, J=$ $\left.16.1 \mathrm{~Hz}, 1 \mathrm{H}, \alpha^{\prime}-\mathrm{H}\right), 7.14\left(\mathrm{~d}, J=1.9 \mathrm{~Hz}, 1 \mathrm{H}, 2^{\prime \prime}-\mathrm{H}\right), 7.27(\mathrm{~d}, J=$ $\left.16.1 \mathrm{~Hz}, 1 \mathrm{H}, \beta^{\prime}-\mathrm{H}\right), 7.38-7.42\left(\mathrm{~m}, 4 \mathrm{H}, 6,3^{\prime}, 4^{\prime}, 5^{\prime}-\mathrm{H}\right), 7.42(\mathrm{~d}, J=$ $15.8 \mathrm{~Hz}, 1 \mathrm{H}, \alpha-\mathrm{H}), 7.54(\mathrm{~d}, J=8.0 \mathrm{~Hz}, 1 \mathrm{H}, 8-\mathrm{H}), 7.61(\mathrm{~d}, J=$ $\left.7.3 \mathrm{~Hz}, 2 \mathrm{H}, 2^{\prime}, 6^{\prime}-\mathrm{H}\right), 7.69(\mathrm{dt}, J=8.0,1.5 \mathrm{~Hz}, 1 \mathrm{H}, 7-\mathrm{H}), 7.71(\mathrm{~d}$, $J=15.8 \mathrm{~Hz}, 1 \mathrm{H}, \beta-\mathrm{H}), 8.25(\mathrm{dd}, J=8.0,1.5 \mathrm{~Hz}, 1 \mathrm{H}, 5-\mathrm{H}) .{ }^{13} \mathrm{C}$ NMR: $\delta=55.9\left(3^{\prime \prime}, 4^{\prime \prime}-\mathrm{OCH}_{3}\right), 109.1\left(\mathrm{C}-2^{\prime \prime}\right), 111.1\left(\mathrm{C}-5^{\prime \prime}\right), 117.2$

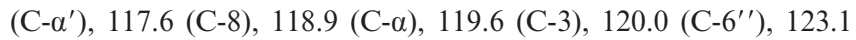
(C-10), 124.7 (C-6), 126.1 (C-5), 127.6 (C-2',6'), 129.0 (C-3',5'), $129.6\left(\mathrm{C}-4^{\prime}\right), 130.7\left(\mathrm{C}-1^{\prime \prime}\right), 133.6(\mathrm{C}-7), 135.6\left(\mathrm{C}-1^{\prime}\right), 136.1\left(\mathrm{C}-\beta^{\prime}\right)$, 136.8 (C- $\beta$ ), 149.1 (C-3"'), 149.2 (C-4" ), 155.1 (C-9), 157.8 (C-2), 177.7 (C-4) ppm. EI-MS: $m / z(\%)=410[\mathrm{M}]^{+\cdot}(100), 333(29), 317$ (6), 289 (3), 273 (27), 261 (4), 215 (6), 202 (10), 189 (5), 151 (31), 138 (10), 121 (11), 92 (10), 77 (8), 63 (5), 51 (5). $\mathrm{C}_{27} \mathrm{H}_{22} \mathrm{O}_{4}$ (410.46): calcd. for C 79.01, H 5.40; found C 78.75, H 5.55.

Synthesis of the 2-Acetylphenyl $(E)$-Cinnamates 10a-i. Method 1: Cinnamoyl chloride $(1.9 \mathrm{~g}, 11.4 \mathrm{mmol})$ was added to a solution of the appropriate acetophenone $(5.7 \mathrm{mmol})$ in dry pyridine $(150 \mathrm{~mL})$. The solution was stirred under nitrogen at room temperature for $2 \mathrm{~h}$. After that period, the solution was poured into ice $(100 \mathrm{~g})$ and water $(100 \mathrm{~mL})$, and the $\mathrm{pH}$ was adjusted to 4 with dilute hydrochloric acid. The mixture was vigorously stirred for $15 \mathrm{~min}$, the precipitate was removed and dissolved in chloroform $(100 \mathrm{~mL})$, and the organic layer was washed with water $(3 \times 100 \mathrm{~mL})$. The solvent was evaporated to dryness, and the residue was purified by silica gel column chromatography with dichloromethane as eluent. The solvent was removed to dryness in each case, and the residue was recrystallised from ethanol to give the desired 2-acetylphenyl $(E)$ cinnamate 10a, 10d, $10 \mathrm{f}$ or $10 \mathrm{~h}$ in good yield.

2-Acetylphenyl (E)-3-Phenylacrylate (10a): This compound showed spectroscopic and analytical data identical to those previously reported. ${ }^{[32]}$ 
2-Acetyl-5-methoxyphenyl (E)-3-Phenylacrylate (10d): Yield $1.47 \mathrm{~g}$ (87\%); m.p. 97-99 ${ }^{\circ} \mathrm{C} .{ }^{1} \mathrm{H}$ NMR: $\delta=2.53$ (s, $3 \mathrm{H}, 2-\mathrm{CH}_{3}$ ), 3.86 (s, $\left.3 \mathrm{H}, 4^{\prime}-\mathrm{OCH}_{3}\right), 6.69$ (d, $\left.J=16.0 \mathrm{~Hz}, 1 \mathrm{H}, \alpha-\mathrm{H}\right), 6.69(\mathrm{~d}, J=2.5 \mathrm{~Hz}$, $\left.1 \mathrm{H}, 3^{\prime}-\mathrm{H}\right), 6.85$ (dd, $\left.J=8.8,2.5 \mathrm{~Hz}, 1 \mathrm{H}, 5^{\prime}-\mathrm{H}\right), 7.42-7.44$ (m, 3 $\left.\mathrm{H}, 3^{\prime \prime}, 4^{\prime \prime}, 5^{\prime \prime}-\mathrm{H}\right), 7.59-7.62\left(\mathrm{~m}, 2 \mathrm{H}, 2^{\prime \prime}, 6^{\prime \prime}-\mathrm{H}\right), 7.88(\mathrm{~d}, J=8.8 \mathrm{~Hz}$, $\left.1 \mathrm{H}, 6^{\prime}-\mathrm{H}\right), 7.91(\mathrm{~d}, J=16.0 \mathrm{~Hz}, 1 \mathrm{H}, \beta-\mathrm{H}) \mathrm{ppm} .{ }^{13} \mathrm{C} \mathrm{NMR}: \delta=$ $29.5\left(2-\mathrm{CH}_{3}\right), 55.7\left(4^{\prime}-\mathrm{OCH}_{3}\right), 109.1\left(\mathrm{C}-3^{\prime}\right), 111.9\left(\mathrm{C}-5^{\prime}\right), 116.8(\mathrm{C}-$ a), $123.5\left(\mathrm{C}-1^{\prime}\right), 128.4\left(\mathrm{C}-2^{\prime \prime} .6^{\prime \prime}\right), 128.9\left(\mathrm{C}-3^{\prime \prime}, 5^{\prime \prime}\right), 130.8\left(\mathrm{C}-4^{\prime \prime}\right)$, $132.3\left(\mathrm{C}-6^{\prime}\right), 134.0\left(\mathrm{C}-1^{\prime \prime}\right), 147.3(\mathrm{C}-\beta), 151.4\left(\mathrm{C}-2^{\prime}\right), 163.7\left(\mathrm{C}-4^{\prime}\right)$, $165.1(\mathrm{C}=\mathrm{O}), 195.8(\mathrm{C}-1)$ ppm. ESI-MS: $m / z(\%)=297[\mathrm{M}+\mathrm{H}]^{+}$ (8), $319[\mathrm{M}+\mathrm{Na}]^{+}(100), 335[\mathrm{M}+\mathrm{K}]^{+}(13), 615[2 \times \mathrm{M}+\mathrm{Na}]^{+}$ (43). $\mathrm{C}_{18} \mathrm{H}_{16} \mathrm{O}_{4}$ (296.32): calcd. for $\mathrm{C} 72.96$, H 5.44; found $\mathrm{C} 72.84$, H 5.57 .

2-Acetyl-3-methoxyphenyl (E)-3-Phenylacrylate (10f): Yield $1.43 \mathrm{~g}$ (85\%); m.p. $101-102{ }^{\circ} \mathrm{C} .{ }^{1} \mathrm{H}$ NMR: $\delta=2.52$ (s, $\left.3 \mathrm{H}, 2-\mathrm{CH}_{3}\right), 3.87$ (s, $\left.3 \mathrm{H}, 6^{\prime}-\mathrm{OCH}_{3}\right), 6.58(\mathrm{~d}, J=16.0 \mathrm{~Hz}, 1 \mathrm{H}, \alpha-\mathrm{H}), 6.81(\mathrm{dd}, J=$ $\left.8.3,0.7 \mathrm{~Hz}, 1 \mathrm{H}, 3^{\prime}-\mathrm{H}\right), 6.85\left(\mathrm{~d}, J=8.3 \mathrm{~Hz}, 1 \mathrm{H}, 5^{\prime}-\mathrm{H}\right), 7.38$ (t, $J$ $\left.=8.3 \mathrm{~Hz}, 1 \mathrm{H}, 4^{\prime}-\mathrm{H}\right), 7.40-7.42\left(\mathrm{~m}, 3 \mathrm{H}, 3^{\prime \prime}, 4^{\prime \prime}, 5^{\prime \prime}-\mathrm{H}\right), 7.55-7.58$ $\left(\mathrm{m}, 2 \mathrm{H}, 2^{\prime \prime}, 6^{\prime \prime}-\mathrm{H}\right), 7.83(\mathrm{~d}, J=16.0 \mathrm{~Hz}, 1 \mathrm{H}, \beta-\mathrm{H}) \mathrm{ppm} \cdot{ }^{13} \mathrm{C}$ NMR: $\delta=31.7\left(2-\mathrm{CH}_{3}\right), 55.9\left(6^{\prime}-\mathrm{OCH}_{3}\right), 108.6\left(\mathrm{C}-5^{\prime}\right), 115.1(\mathrm{C}-$ $\left.3^{\prime}\right), 116.6(\mathrm{C}-\alpha), 124.5\left(\mathrm{C}-1^{\prime}\right), 128.4\left(\mathrm{C}-2^{\prime \prime}, 6^{\prime \prime}\right), 128.9\left(\mathrm{C}-3^{\prime \prime}, 5^{\prime \prime}\right)$, $130.8\left(\mathrm{C}-4^{\prime \prime}\right), 130.9\left(\mathrm{C}-4^{\prime}\right), 134.0\left(\mathrm{C}-1^{\prime \prime}\right), 147.2(\mathrm{C}-\beta), 147.6\left(\mathrm{C}-2^{\prime}\right)$, $157.3\left(\mathrm{C}-6^{\prime}\right), 165.1(\mathrm{C}=\mathrm{O}), 200.7(\mathrm{C}-1) \mathrm{ppm}$. ESI-MS: $m / z(\%)=$ $297[\mathrm{M}+\mathrm{H}]^{+}(6), 319[\mathrm{M}+\mathrm{Na}]^{+}(100), 335[\mathrm{M}+\mathrm{K}]^{+}(16), 615$ $[2 \mathrm{M}+\mathrm{Na}]^{+}(89) \cdot \mathrm{C}_{18} \mathrm{H}_{16} \mathrm{O}_{4}$ (296.32): calcd. for C 72.96, H 5.44; found C 73.36, H 5.47 .

2-Acetyl-3,5-dimethoxyphenyl (E)-3-Phenylacrylate (10h): Yield 1.75 g $(94 \%)$; m.p. $97-98{ }^{\circ} \mathrm{C} .{ }^{1} \mathrm{H}$ NMR: $\delta=2.49$ (s, $\left.3 \mathrm{H}, 2-\mathrm{CH}_{3}\right)$, $3.82\left(\mathrm{~s}, 3 \mathrm{H}, 4^{\prime}-\mathrm{OCH}_{3}\right), 3.86\left(\mathrm{~s}, 3 \mathrm{H}, 6^{\prime}-\mathrm{OCH}_{3}\right), 6.31$ (d, $J=2.2 \mathrm{~Hz}$, $\left.1 \mathrm{H}, 3^{\prime}-\mathrm{H}\right), 6.39\left(\mathrm{~d}, J=2.2 \mathrm{~Hz}, 1 \mathrm{H}, 5^{\prime}-\mathrm{H}\right), 6.59(\mathrm{~d}, J=16.0 \mathrm{~Hz}$, $1 \mathrm{H}, \alpha-\mathrm{H}), 7.40-7.42\left(\mathrm{~m}, 3 \mathrm{H}, 3^{\prime \prime}, 4^{\prime \prime}, 5^{\prime \prime}-\mathrm{H}\right), 7.56-7.59(\mathrm{~m}, 2 \mathrm{H}$, $\left.2^{\prime \prime}, 6^{\prime \prime}-\mathrm{H}\right), 7.83(\mathrm{~d}, J=16.0 \mathrm{~Hz}, 1 \mathrm{H}, \beta-\mathrm{H}) \mathrm{ppm} .{ }^{13} \mathrm{C}$ NMR: $\delta=$ $31.9\left(2-\mathrm{CH}_{3}\right), 55.6\left(4^{\prime}-\mathrm{OCH}_{3}\right), 55.9\left(6^{\prime}-\mathrm{OCH}_{3}\right), 96.5\left(\mathrm{C}-5^{\prime}\right), 99.9$ $\left(\mathrm{C}-3^{\prime}\right), 116.7(\mathrm{C}-\alpha), 117.3\left(\mathrm{C}-1^{\prime}\right), 128.4\left(\mathrm{C}-2^{\prime \prime}, 6^{\prime \prime}\right), 128.9\left(\mathrm{C}-3^{\prime \prime}, 5^{\prime \prime}\right)$, 130.7 (C-4" $), 134.0$ (C-1' $), 147.1$ (C- $\beta), 149.5$ (C-2'), 159.1 (C-6'), $162.2\left(\mathrm{C}-4^{\prime}\right), 165.2(\mathrm{C}=\mathrm{O}), 199.5(\mathrm{C}-1) \mathrm{ppm}$. ESI-MS: $m / z(\%)=$ $327[\mathrm{M}+\mathrm{H}]^{+}(14), 349[\mathrm{M}+\mathrm{Na}]^{+}(73), 365[\mathrm{M}+\mathrm{K}]^{+}(12), 675[2$ $\mathrm{M}+\mathrm{Na}]^{+}(100) \cdot \mathrm{C}_{19} \mathrm{H}_{18} \mathrm{O}_{5}$ (326.34): calcd. for $\mathrm{C}$ 69.93, H 5.56; found C 69.81, H 5.62 .

Method 2: The appropriate cinnamic acid $(16.8 \mathrm{mmol})$ and phosphorus oxychloride $(4.2 \mathrm{~mL}, 45.9 \mathrm{mmol})$ were added to a solution of the appropriate acetophenone $(8.6 \mathrm{mmol})$ in dry pyridine $(100 \mathrm{~mL})$. The solution was stirred at $60{ }^{\circ} \mathrm{C}$ for $2 \mathrm{~h}$, and after that the solution was poured into ice $(50 \mathrm{~g})$ and water $(100 \mathrm{~mL})$ and the $\mathrm{pH}$ was adjusted to 4 with hydrochloric acid. The precipitate was removed by filtration, the residue was dissolved with chloroform $(100 \mathrm{~mL})$, and the organic layer was washed with water $(3 \times 100 \mathrm{~mL})$. The solvent was evaporated, and the residue was purified by silica gel column chromatography with light petroleum/ dichloromethane 3:7 as eluent. The solvent was removed to dryness in each case, and the residue was recrystallised from ethanol to give the desired 2-acetylphenyl (E)-cinnamates $10 b, 10 c, 10 e, 10 \mathrm{~g}$ or $10 \mathbf{i}$ in good yield.

2-Acetylphenyl (E)-2-(4-Methoxyphenyl)acrylate (10b): This compound showed spectroscopic and analytical data identical to those previously reported. ${ }^{[32]}$

2-Acetylphenyl (E)-2-(3,4-Dimethoxyphenyl)acrylate (10c): Yield 1.96 g $(70 \%)$; m.p. $97-99{ }^{\circ} \mathrm{C} .{ }^{1} \mathrm{H}$ NMR: $\delta=2.58$ (s, $\left.3 \mathrm{H}, 2-\mathrm{CH}_{3}\right)$, 3.94 (s, $\left.6 \mathrm{H}, 3^{\prime \prime}, 4^{\prime \prime}-\mathrm{OCH}_{3}\right), 6.55(\mathrm{~d}, J=16.0 \mathrm{~Hz}, 1 \mathrm{H}, \alpha-\mathrm{H}), 6.90$ (d, $\left.J=8.3 \mathrm{~Hz}, 1 \mathrm{H}, 5^{\prime \prime}-\mathrm{H}\right), 7.13$ (d, $\left.J=1.9 \mathrm{~Hz}, 1 \mathrm{H}, 2^{\prime \prime}-\mathrm{H}\right), 7.18$ $\left(\mathrm{dd}, J=8.3,1.9 \mathrm{~Hz}, 1 \mathrm{H}, 6^{\prime \prime}-\mathrm{H}\right), 7.19(\mathrm{dd}, J=7.9,1.2 \mathrm{~Hz}, 1 \mathrm{H}$, $\left.3^{\prime}-\mathrm{H}\right), 7.35\left(\mathrm{dt}, J=7.9,1.2 \mathrm{~Hz}, 1 \mathrm{H}, 5^{\prime}-\mathrm{H}\right), 7.57$ (dt, $J=7.9,1.7 \mathrm{~Hz}$,
$1 \mathrm{H}, 4-\mathrm{H}), 7.85$ (dd, $J=7.9,1.7 \mathrm{~Hz}, 1 \mathrm{H}, 6-\mathrm{H}), 7.85$ (d, $J=$ $16.0 \mathrm{~Hz}, 1 \mathrm{H}, \beta-\mathrm{H}) \mathrm{ppm} .{ }^{13} \mathrm{C} \mathrm{NMR}: \delta=29.8\left(2-\mathrm{CH}_{3}\right), 55.9$ and $56.0\left(3^{\prime \prime}, 4^{\prime \prime}-\mathrm{OCH}_{3}\right), 109.6\left(\mathrm{C}-2^{\prime \prime}\right), 110.9\left(\mathrm{C}-5^{\prime \prime}\right), 114.2(\mathrm{C}-\alpha), 123.3$ $\left(\mathrm{C}-6^{\prime \prime}\right), 123.8\left(\mathrm{C}-3^{\prime}\right), 126.0\left(\mathrm{C}-5^{\prime}\right), 126.9\left(\mathrm{C}-1^{\prime \prime}\right), 130.1\left(\mathrm{C}-6^{\prime}\right), 131.4$ (C-1'), 133.3 (C-4'), 147.3 (C- $\beta$ ), 149.2 (C-2', C-3' '), 151.6 (C-4' '), $165.5(\mathrm{C}=\mathrm{O}), 197.9(\mathrm{C}-1) \mathrm{ppm}$. ESI-MS: $\mathrm{m} / z(\%)=349[\mathrm{M}+$ $\mathrm{Na}^{+}(100), 365[\mathrm{M}+\mathrm{K}]^{+}(17), 675[2 \mathrm{M}+\mathrm{Na}]^{+}(86) \cdot \mathrm{C}_{19} \mathrm{H}_{18} \mathrm{O}_{5}$ (326.34): calcd. for C 69.93, H 5.56; found C 69.56, H 5.69.

2-Acetyl-5-methoxyphenyl (E)-2-(3,4-Dimethoxyphenyl)acrylate (10e): Yield $2.05 \mathrm{~g}(67 \%)$; m.p. $142-143{ }^{\circ} \mathrm{C} .{ }^{1} \mathrm{H}$ NMR: $\delta=2.53(\mathrm{~s}$, $\left.3 \mathrm{H}, 2-\mathrm{CH}_{3}\right), 3.87\left(\mathrm{~s}, 3 \mathrm{H}, 4^{\prime}-\mathrm{OCH}_{3}\right), 3.94\left(\mathrm{~s}, 6 \mathrm{H}, 3^{\prime \prime}, 4^{\prime \prime}-\mathrm{OCH}_{3}\right)$, $6.56(\mathrm{~d}, J=15.9 \mathrm{~Hz}, 1 \mathrm{H}, \alpha-\mathrm{H}), 6.68\left(\mathrm{~d}, J=2.5 \mathrm{~Hz}, 1 \mathrm{H}, 3^{\prime}-\mathrm{H}\right)$, $6.85\left(\mathrm{dd}, J=8.8,2.5 \mathrm{~Hz}, 1 \mathrm{H}, 5^{\prime}-\mathrm{H}\right), 6.90$ (d, $J=8.3 \mathrm{~Hz}, 1 \mathrm{H}, 5^{\prime \prime}-$ $\mathrm{H}), 7.13\left(\mathrm{~d}, J=1.9 \mathrm{~Hz}, 1 \mathrm{H}, 2^{\prime \prime}-\mathrm{H}\right), 7.18(\mathrm{dd}, J=8.3,1.9 \mathrm{~Hz}, 1$ $\left.\mathrm{H}, 6^{\prime \prime}-\mathrm{H}\right), 7.85(\mathrm{~d}, J=15.9 \mathrm{~Hz}, 1 \mathrm{H}, \beta-\mathrm{H}), 7.88(\mathrm{~d}, J=8.8 \mathrm{~Hz}, 1$ $\left.\mathrm{H}, 6^{\prime}-\mathrm{H}\right)$ ppm. ${ }^{13} \mathrm{C}$ NMR: $\delta=29.6\left(2-\mathrm{CH}_{3}\right), 55.7\left(4^{\prime}-\mathrm{OCH}_{3}\right), 55.9$ and $56.0\left(3^{\prime \prime}, 4^{\prime \prime}-\mathrm{OCH}_{3}\right), 109.1\left(\mathrm{C}-3^{\prime}\right), 109.7\left(\mathrm{C}-2^{\prime \prime}\right), 110.9\left(\mathrm{C}-5^{\prime \prime}\right)$, $111.8\left(\mathrm{C}-5^{\prime}\right), 114.4(\mathrm{C}-\alpha), 123.3\left(\mathrm{C}-6^{\prime \prime}\right), 123.6\left(\mathrm{C}-1^{\prime}\right), 127.0\left(\mathrm{C}-1^{\prime \prime}\right)$, $132.3\left(\mathrm{C}-6^{\prime}\right), 147.3(\mathrm{C}-\beta), 149.2\left(\mathrm{C}-3^{\prime \prime}\right), 151.50$ and $151.54\left(\mathrm{C}-2^{\prime}\right.$, C-4' $), 163.7\left(\mathrm{C}-4^{\prime}\right), 165.4(\mathrm{C}=\mathrm{O}), 195.9(\mathrm{C}-1) \mathrm{ppm}$. ESI-MS: $\mathrm{m} / \mathrm{z}$ $(\%)=379[\mathrm{M}+\mathrm{Na}]^{+}(92), 395[\mathrm{M}+\mathrm{K}]^{+}(15), 735[2 \mathrm{M}+\mathrm{Na}]^{+}$ (100), $751[2 \mathrm{M}+\mathrm{K}]^{+}(4), 1091[3 \mathrm{M}+\mathrm{Na}]^{+}$(4). $\mathrm{C}_{20} \mathrm{H}_{20} \mathrm{O}_{6}(356.37)$ : calcd. for C 67.41, H 5.66; found C 67.32, H 5.94.

2-Acetyl-3-methoxyphenyl ( $E)$-2-(3,4-Dimethoxyphenyl)acrylate (10g): Yield 1.96 g $(64 \%)$; m.p. $86-88^{\circ} \mathrm{C} .{ }^{1} \mathrm{H}$ NMR: $\delta=2.52$ (s, 3 $\left.\mathrm{H}, 2-\mathrm{CH}_{3}\right), 3.88\left(\mathrm{~s}, 3 \mathrm{H}, 6^{\prime}-\mathrm{OCH}_{3}\right), 3.93$ and $3.94\left(2 \times \mathrm{s}, 6 \mathrm{H}, 3^{\prime \prime}, 4^{\prime \prime}{ }^{\prime}-\right.$ $\left.\mathrm{OCH}_{3}\right), 6.45(\mathrm{~d}, J=15.8 \mathrm{~Hz}, 1 \mathrm{H}, \alpha-\mathrm{H}), 6.81(\mathrm{dd}, J=8.3,0.7 \mathrm{~Hz}$, $\left.1 \mathrm{H}, 3^{\prime}-\mathrm{H}\right), 6.85$ (d, $\left.J=8.4 \mathrm{~Hz}, 1 \mathrm{H}, 5^{\prime}-\mathrm{H}\right), 6.89$ (d, $J=8.3 \mathrm{~Hz}, 1$ $\left.\mathrm{H}, 5^{\prime \prime}-\mathrm{H}\right), 7.10\left(\mathrm{~d}, J=2.0 \mathrm{~Hz}, 1 \mathrm{H}, 2^{\prime \prime}-\mathrm{H}\right), 7.15(\mathrm{dd}, J=8.3,2.0 \mathrm{~Hz}$, $\left.1 \mathrm{H}, 6^{\prime \prime}-\mathrm{H}\right), 7.38\left(\mathrm{dd}, J=8.4,8.3 \mathrm{~Hz}, 1 \mathrm{H}, 4^{\prime}-\mathrm{H}\right), 7.78(\mathrm{~d}, J=$ $15.8 \mathrm{~Hz}, 1 \mathrm{H}, \beta-\mathrm{H})$ ppm. ${ }^{13} \mathrm{C}$ NMR: $\delta=31.7\left(2-\mathrm{CH}_{3}\right), 55.9$ and $56.0\left(6^{\prime}, 3^{\prime \prime}, 4^{\prime \prime}-\mathrm{OCH}_{3}\right), 108.6\left(\mathrm{C}-5^{\prime}\right), 109.6\left(\mathrm{C}-2^{\prime \prime}\right), 110.9\left(\mathrm{C}-5^{\prime \prime}\right)$, $114.2(\mathrm{C}-\alpha), 115.2\left(\mathrm{C}-3^{\prime}\right), 123.3\left(\mathrm{C}-6^{\prime \prime}\right), 124.6\left(\mathrm{C}-1^{\prime}\right), 127.0\left(\mathrm{C}-1^{\prime \prime}\right)$, 130.9 (C-4'), 147.1 (C- $\beta$ ), 147.7 (C-2'), 149.2 (C-3' $), 151.5$ (C-4' $\left.{ }^{\prime \prime}\right)$, $157.3\left(\mathrm{C}-6^{\prime}\right), 165.3(\mathrm{C}=\mathrm{O}), 200.9(\mathrm{C}-1) \mathrm{ppm}$. ESI-MS: $\mathrm{m} / \mathrm{z}(\%)=$ $357[\mathrm{M}+\mathrm{H}]^{+}(15), 379[\mathrm{M}+\mathrm{Na}]^{+}(53), 395[\mathrm{M}+\mathrm{K}]^{+}(11), 753$ $[2 \mathrm{M}+\mathrm{Na}]^{+}$(31). $\mathrm{C}_{20} \mathrm{H}_{20} \mathrm{O}_{6}$ (356.37): calcd. for $\mathrm{C}$ 67.41, $\mathrm{H}$ 5.66; found C 67.55, H 5.74.

2-Acetyl-3,5-dimethoxyphenyl (E)-2-(3,4-Dimethoxyphenyl)acrylate (10i): Yield $1.66 \mathrm{~g}(50 \%)$; m.p. $140-142{ }^{\circ} \mathrm{C} .{ }^{1} \mathrm{H}$ NMR: $\delta=2.49$ (s, $\left.3 \mathrm{H}, 2-\mathrm{CH}_{3}\right), 3.82\left(\mathrm{~s}, 3 \mathrm{H}, 4^{\prime}-\mathrm{OCH}_{3}\right), 3.85$ (s, $\left.3 \mathrm{H}, 6^{\prime}-\mathrm{OCH}_{3}\right), 3.93$ (s, $\left.6 \mathrm{H}, 3^{\prime \prime}, 4^{\prime \prime}-\mathrm{OCH}_{3}\right), 6.31\left(\mathrm{~d}, J=2.2 \mathrm{~Hz}, 1 \mathrm{H}, 3^{\prime}-\mathrm{H}\right), 6.38(\mathrm{~d}, J$ $\left.=2.2 \mathrm{~Hz}, 1 \mathrm{H}, 5^{\prime}-\mathrm{H}\right), 6.46(\mathrm{~d}, J=15.9 \mathrm{~Hz}, 1 \mathrm{H}, \alpha-\mathrm{H}), 6.88(\mathrm{~d}, J=$ $\left.8.3 \mathrm{~Hz}, 1 \mathrm{H}, 5^{\prime \prime}-\mathrm{H}\right), 7.10$ (d, $\left.J=2.0 \mathrm{~Hz}, 1 \mathrm{H}, 2^{\prime \prime}-\mathrm{H}\right), 7.15(\mathrm{dd}, J=$ 8.3, $\left.2.0 \mathrm{~Hz}, 1 \mathrm{H}, 6^{\prime \prime}-\mathrm{H}\right), 7.78(\mathrm{~d}, J=15.9 \mathrm{~Hz}, 1 \mathrm{H}, \beta-\mathrm{H}) \mathrm{ppm} .{ }^{13} \mathrm{C}$ NMR: $\delta=31.8\left(2-\mathrm{CH}_{3}\right), 55.6\left(4^{\prime}-\mathrm{OCH}_{3}\right), 55.8\left(3^{\prime \prime}, 4^{\prime \prime}-\mathrm{OCH}_{3}\right), 55.9$ $\left(6^{\prime}-\mathrm{OCH}_{3}\right), 96.4\left(\mathrm{C}-5^{\prime}\right), 100.0\left(\mathrm{C}-3^{\prime}\right), 109.6\left(\mathrm{C}-2^{\prime \prime}\right), 110.9\left(\mathrm{C}-5^{\prime \prime}\right)$, $114.3(\mathrm{C}-\alpha), 117.3\left(\mathrm{C}-1^{\prime}\right), 123.2\left(\mathrm{C}-6^{\prime \prime}\right), 127.0\left(\mathrm{C}-1^{\prime \prime}\right), 147.0(\mathrm{C}-\beta)$, $149.1\left(\mathrm{C}-3^{\prime \prime}\right), 149.6\left(\mathrm{C}-2^{\prime}\right), 151.4\left(\mathrm{C}-4^{\prime \prime}\right), 159.0\left(\mathrm{C}-6^{\prime}\right), 162.1$ (C$\left.4^{\prime}\right), 165.4(\mathrm{C}=\mathrm{O}), 199.6(\mathrm{C}-1)$ ppm. ESI-MS: $m / z(\%)=387[\mathrm{M}+$ $\mathrm{H}]^{+}(6), 409[\mathrm{M}+\mathrm{Na}]^{+}(89), 425[\mathrm{M}+\mathrm{K}]^{+}(12), 795[2 \mathrm{M}+\mathrm{Na}]^{+}$ (100), $811[2 \mathrm{M}+\mathrm{K}]^{+}$(5). $\mathrm{C}_{21} \mathrm{H}_{22} \mathrm{O}_{7}$ (386.40): calcd. for $\mathrm{C} 65.28$, H 5.74; found C 65.60, H 5.71.

Synthesis of the 5-Aryl-3-hydroxy-1-(2-hydroxyphenyl)penta-2,4dien-1-ones 11a-i: Potassium hydroxide (powder, $1.4 \mathrm{~g}, 25 \mathrm{mmol}$ ) was added to a solution of the appropriate 2-acetylphenyl cinnamate $(\mathbf{1 0 a}-\mathbf{i}, 5 \mathrm{mmol})$ in dimethyl sulfoxide $(30 \mathrm{~mL})$. The solution was stirred at room temperature for $2 \mathrm{~h}$. After that period, the solution was poured into ice $(50 \mathrm{~g})$ and water $(100 \mathrm{~mL})$, and the $\mathrm{pH}$ was adjusted to 4 with dilute hydrochloric acid. The obtained solid was removed by filtration, dissolved in chloroform $(80 \mathrm{~mL})$ and washed with water $(2 \times 100 \mathrm{~mL})$. The solvent was evaporated to 
dryness in each case, and the residue was recrystallised from ethanol to give the expected 5-aryl-3-hydroxy-1-(2-hydroxyphenyl)-2,4pentadien-1-one (11a-i) in good yield.

5-Aryl-3-hydroxy-1-(2-hydroxyphenyl)penta-2,4-dien-1-ones 11a and 11b: These compounds showed spectroscopic and analytical data identical to those previously reported. ${ }^{[32]}$

3-Hydroxy-1-(2-hydroxyphenyl)-5-(3,4-dimethoxyphenyl)penta-2,4dien-1-one (11c): Yield $1.19 \mathrm{~g}(73 \%) ;$ m.p. $136-138^{\circ} \mathrm{C} .{ }^{1} \mathrm{H}$ NMR: $\delta$ $=3.93\left(\mathrm{~s}, 3 \mathrm{H}, 4^{\prime \prime}-\mathrm{OCH}_{3}\right), 3.95\left(\mathrm{~s}, 3 \mathrm{H}, 3^{\prime \prime}-\mathrm{OCH}_{3}\right), 6.31(\mathrm{~s}, 1 \mathrm{H}, 2-$ $\mathrm{H}), 6.47$ (brd, $J=15.8 \mathrm{~Hz}, 1 \mathrm{H}, 4-\mathrm{H}), 6.89$ (ddd, $J=8.3,8.1$, $\left.1.0 \mathrm{~Hz}, 1 \mathrm{H}, 5^{\prime}-\mathrm{H}\right), 6.89$ (d, $\left.J=8.3 \mathrm{~Hz}, 1 \mathrm{H}, 5^{\prime \prime}-\mathrm{H}\right), 6.99$ (dd, $J=$ 7.7, $\left.1.0 \mathrm{~Hz}, 1 \mathrm{H}, 3^{\prime}-\mathrm{H}\right), 7.08$ (d, $\left.J=1.9 \mathrm{~Hz}, 1 \mathrm{H}, 2^{\prime \prime}-\mathrm{H}\right), 7.15$ (dd, $\left.J=8.3,1.9 \mathrm{~Hz}, 1 \mathrm{H}, 6^{\prime \prime}-\mathrm{H}\right), 7.45$ (ddd, $J=8.1,7.7,1.5 \mathrm{~Hz}, 1 \mathrm{H}$, $\left.4^{\prime}-\mathrm{H}\right), 7.61(\mathrm{~d}, J=15.8 \mathrm{~Hz}, 1 \mathrm{H}, 5-\mathrm{H}), 7.70(\mathrm{dd}, J=8.3,1.5 \mathrm{~Hz}, 1$ $\left.\mathrm{H}, 6^{\prime}-\mathrm{H}\right), 12.28$ (s, $\left.1 \mathrm{H}, 2^{\prime}-\mathrm{OH}\right), 14.74(\mathrm{~d}, J=0.8 \mathrm{~Hz}, 1 \mathrm{H}, 3-$ $\mathrm{O} H)$ ppm. ${ }^{13} \mathrm{C}$ NMR: $\delta=55.9\left(3^{\prime \prime}-\mathrm{OCH}_{3}\right), 56.0\left(4^{\prime \prime}-\mathrm{OCH}_{3}\right), 96.5$ (C-2), 109.5 (C-2' ), 111.1 (C-5' $), 118.7$ (C-3'), 118.9 (C-5'), 119.1 $\left(\mathrm{C}-1^{\prime}\right), 119.8$ (C-4), $122.6\left(\mathrm{C}-6^{\prime \prime}\right), 127.9\left(\mathrm{C}-1^{\prime \prime}\right), 128.4\left(\mathrm{C}-6^{\prime}\right), 135.6$ (C-4') 139.9 (C-5), 149.2 (C-3' '), 151.1 (C-4' '), 162.5 (C-2'), 175.0 (C-3), 195.6 (C-1) ppm. ESI-MS: $m / z(\%)=327[\mathrm{M}+\mathrm{H}]^{+}(35), 349$ $[\mathrm{M}+\mathrm{Na}]^{+}$(25). $\mathrm{C}_{19} \mathrm{H}_{18} \mathrm{O}_{5}$ (326.34): calcd. for $\mathrm{C}$ 69.93, $\mathrm{H}$ 5.56; found $\mathrm{C} 69.54, \mathrm{H} 5.74 \%$.

3-Hydroxy-1-(2-hydroxy-4-methoxyphenyl)-5-phenylpenta-2,4-dien1-one (11d): Yield $1.20 \mathrm{~g}(81 \%)$; m.p. $125-127{ }^{\circ} \mathrm{C} .{ }^{1} \mathrm{H}$ NMR: $\delta=$ 3.82 (s, $\left.3 \mathrm{H}, 4^{\prime}-\mathrm{OCH}_{3}\right), 6.18$ (s, $\left.1 \mathrm{H}, 2-\mathrm{H}\right), 6.43$ (brs, $\left.1 \mathrm{H}, 3^{\prime}-\mathrm{H}\right)$, $6.45\left(\mathrm{dd}, J=8.7,2.5 \mathrm{~Hz}, 1 \mathrm{H}, 5^{\prime}-\mathrm{H}\right), 6.56(\mathrm{dd}, J=15.9,0.9 \mathrm{~Hz}, 1$ $\mathrm{H}, 4-\mathrm{H}), 7.35-7.41\left(\mathrm{~m}, 3 \mathrm{H}, 3^{\prime \prime}, 4^{\prime \prime}, 5^{\prime \prime}-\mathrm{H}\right), 7.52-7.55$ (m, $2 \mathrm{H}$, $\left.2^{\prime \prime}, 6^{\prime \prime}-\mathrm{H}\right), 7.59$ (d, $\left.J=8.7 \mathrm{~Hz}, 1 \mathrm{H}, 6^{\prime}-\mathrm{H}\right), 7.59$ (d, $J=15.9 \mathrm{~Hz}, 1$ $\mathrm{H}, 5-\mathrm{H}), 12.70\left(\mathrm{~s}, 1 \mathrm{H}, 2^{\prime}-\mathrm{OH}\right), 14.51(\mathrm{~d}, J=0.9 \mathrm{~Hz}, 1 \mathrm{H}, 3-$ $\mathrm{O} H)$ ppm. ${ }^{13} \mathrm{C}$ NMR: $\delta=55.5\left(4^{\prime}-\mathrm{OCH}_{3}\right), 96.8(\mathrm{C}-2), 101.2\left(\mathrm{C}-3^{\prime}\right)$, $107.9\left(\mathrm{C}-5^{\prime}\right), 112.7\left(\mathrm{C}-1^{\prime}\right), 122.3(\mathrm{C}-4), 127.9\left(\mathrm{C}-2^{\prime \prime}, 6^{\prime \prime}\right), 128.9(\mathrm{C}-$ $\left.3^{\prime \prime}, 5^{\prime \prime}\right), 129.8\left(\mathrm{C}-4^{\prime \prime}\right), 130.1\left(\mathrm{C}-6^{\prime}\right), 135.1\left(\mathrm{C}-1^{\prime \prime}\right), 139.0$ (C-5), 165.4 $\left(\mathrm{C}-4^{\prime}\right), 165.8\left(\mathrm{C}-2^{\prime}\right), 173.0(\mathrm{C}-3), 194.6(\mathrm{C}-1) \mathrm{ppm}$. ESI-MS: $\mathrm{m} / \mathrm{z}$ $(\%)=297[\mathrm{M}+\mathrm{H}]^{+}(15), 319[\mathrm{M}+\mathrm{Na}]^{+}(17) . \mathrm{C}_{18} \mathrm{H}_{16} \mathrm{O}_{4}$ (296.32): calcd. for C 72.96, H 5.44; found C 72.74, H 5.47.

5-(3,4-Dimethoxyphenyl)-3-hydroxy-1-(2-hydroxy-4-methoxyphenyl)penta-2,4-dien-1-one (11e): Yield $1.21 \mathrm{~g}(68 \%)$; m.p. $149-151^{\circ} \mathrm{C} .{ }^{1} \mathrm{H}$ NMR: $\delta=3.84\left(\mathrm{~s}, 3 \mathrm{H}, 4^{\prime}-\mathrm{OCH}_{3}\right), 3.93\left(\mathrm{~s}, 3 \mathrm{H}, 4^{\prime \prime}{ }^{\prime}-\mathrm{OCH}_{3}\right), 3.95$ (s, $3 \mathrm{H}, 3^{\prime \prime}-\mathrm{OCH}_{3}$ ), 6.18 (s, $\left.1 \mathrm{H}, 2-\mathrm{H}\right), 6.44$ (brs, $\left.1 \mathrm{H}, 3^{\prime}-\mathrm{H}\right), 6.45$ (dd, $J=15.4,1.0 \mathrm{~Hz}, 1 \mathrm{H}, 4-\mathrm{H}), 6.44-6.47$ (m, $\left.1 \mathrm{H}, 5^{\prime}-\mathrm{H}\right), 6.89$ (d, $\left.J=8.3 \mathrm{~Hz}, 1 \mathrm{H}, 5^{\prime \prime}-\mathrm{H}\right), 7.07\left(\mathrm{~d}, J=1.9 \mathrm{~Hz}, 1 \mathrm{H}, 2^{\prime \prime}-\mathrm{H}\right), 7.14(\mathrm{dd}$, $\left.J=8.3,1.9 \mathrm{~Hz}, 1 \mathrm{H}, 6^{\prime \prime}-\mathrm{H}\right), 7.56(\mathrm{~d}, J=15.4 \mathrm{~Hz}, 1 \mathrm{H}, 5-\mathrm{H}), 7.59$ $7.62\left(\mathrm{~m}, 1 \mathrm{H}, 6^{\prime}-\mathrm{H}\right), 12.74$ (s, $\left.1 \mathrm{H}, 2^{\prime}-\mathrm{OH}\right), 14.59$ (d, $J=1.0 \mathrm{~Hz}, 1$ $\mathrm{H}, 3-\mathrm{OH})$ ppm. ${ }^{13} \mathrm{C} \mathrm{NMR}: \delta=55.5\left(4^{\prime}-\mathrm{OCH}_{3}\right), 55.9\left(3^{\prime \prime}-\mathrm{OCH}_{3}\right)$, $56.0\left(4^{\prime \prime}-\mathrm{OCH}_{3}\right), 96.2(\mathrm{C}-2), 101.2\left(\mathrm{C}-3^{\prime}\right), 107.8\left(\mathrm{C}-5^{\prime}\right), 109.5(\mathrm{C}-$ $\left.2^{\prime \prime}\right), 111.1\left(\mathrm{C}-5^{\prime \prime}\right), 112.7\left(\mathrm{C}-1^{\prime}\right), 120.1$ (C-4), $122.4\left(\mathrm{C}-6^{\prime \prime}\right), 128.1$ $\left(\mathrm{C}-1^{\prime \prime}\right), 130.0\left(\mathrm{C}-6^{\prime}\right), 139.1(\mathrm{C}-5), 149.2\left(\mathrm{C}-3^{\prime \prime}\right), 150.8$ (C-4"'), 165.3 $\left(\mathrm{C}-2^{\prime}\right), 165.7\left(\mathrm{C}-4^{\prime}\right), 173.6$ (C-3), 194.3 (C-1) ppm. ESI-MS: $m / z$ $(\%)=357[\mathrm{M}+\mathrm{H}]^{+}(18), 379[\mathrm{M}+\mathrm{Na}]^{+}(27), 735[2 \mathrm{M}+\mathrm{Na}]^{+}$ (7). $\mathrm{C}_{20} \mathrm{H}_{20} \mathrm{O}_{6}$ (356.37): calcd. for $\mathrm{C} 67.41$, $\mathrm{H}$ 5.66; found $\mathrm{C} 67.85$, H 5.78 .

3-Hydroxy-1-(2-hydroxy-6-methoxyphenyl)-5-phenylpenta-2,4-dien1-one (11f): Yield 1.38 g (93\%); m.p. $123-125{ }^{\circ} \mathrm{C} .{ }^{1} \mathrm{H}$ NMR: $\delta=$ 3.93 (s, $\left.3 \mathrm{H}, 6^{\prime}-\mathrm{OCH}_{3}\right), 6.42\left(\mathrm{dd}, J=8.2,0.7 \mathrm{~Hz}, 1 \mathrm{H}, 5^{\prime}-\mathrm{H}\right), 6.59$ (dd, $J=15.8,1.1 \mathrm{~Hz}, 1 \mathrm{H}, 4-\mathrm{H}), 6.60$ (dd, $J=8.4,0.7 \mathrm{~Hz}, 1 \mathrm{H}, 3^{\prime}-$ H), 6.80 (s, $1 \mathrm{H}, 2-\mathrm{H}), 7.32$ (dd, $\left.J=8.4,8.2 \mathrm{~Hz}, 1 \mathrm{H}, 4^{\prime}-\mathrm{H}\right), 7.36-$ 7.42 (m, $\left.3 \mathrm{H}, 3^{\prime \prime}, 4^{\prime \prime}, 5^{\prime \prime}-\mathrm{H}\right), 7.55-7.58$ (m, $\left.2 \mathrm{H}, 2^{\prime \prime}, 6^{\prime \prime}-\mathrm{H}\right), 7.63$ (d, $J=15.8 \mathrm{~Hz}, 1 \mathrm{H}, 5-\mathrm{H}), 12.73\left(\mathrm{~s}, 1 \mathrm{H}, 2^{\prime}-\mathrm{OH}\right), 14.88(\mathrm{~d}, J=1.1 \mathrm{~Hz}$, $1 \mathrm{H}, 3-\mathrm{OH})$ ppm. ${ }^{13} \mathrm{C}$ NMR: $\delta=55.8\left(6^{\prime}-\mathrm{OCH}_{3}\right), 101.7\left(\mathrm{C}-5^{\prime}\right)$,

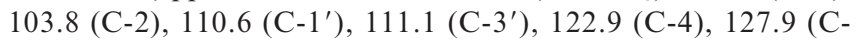
$\left.2^{\prime \prime}, 6^{\prime \prime}\right), 128.9\left(\mathrm{C}-3^{\prime \prime}, 5^{\prime \prime}\right), 129.9\left(\mathrm{C}-4^{\prime \prime}\right), 135.1\left(\mathrm{C}-4^{\prime}\right), 135.2\left(\mathrm{C}-1^{\prime \prime}\right)$, 139.2 (C-5), 160.3 (C-6'), 164.1 (C-2'), 174.3 (C-3), 195.2 (C-
1) ppm. ESI-MS: $m / z(\%)=397[\mathrm{M}+\mathrm{H}]^{+}(22), 319[\mathrm{M}+\mathrm{Na}]^{+}$ (18). $\mathrm{C}_{18} \mathrm{H}_{16} \mathrm{O}_{4}$ (296.32): calcd. for $\mathrm{C} 72.96, \mathrm{H}$ 5.44; found $\mathrm{C} 72.86$, H 5.44.

5-(3,4-Dimethoxyphenyl)-3-hydroxy-1-(2-hydroxy-6-methoxyphenyl)penta-2,4-dien-1-one (11g): Yield $1.16 \mathrm{~g}(65 \%)$; m.p. $123-125^{\circ} \mathrm{C} .{ }^{1} \mathrm{H}$ NMR: $\delta=3.929,3.932$ and $3.95\left(3 \times \mathrm{s}, 9 \mathrm{H}, 6^{\prime}, 3^{\prime \prime}, 4^{\prime \prime}{ }^{\prime}-\mathrm{OCH}_{3}\right), 6.41$ (d, $\left.J=8.2 \mathrm{~Hz}, 1 \mathrm{H}, 5^{\prime}-\mathrm{H}\right), 6.45$ (brd, $\left.J=15.0 \mathrm{~Hz}, 1 \mathrm{H}, 4-\mathrm{H}\right), 6.59$ (dd, $\left.J=8.3,0.8 \mathrm{~Hz}, 1 \mathrm{H}, 3^{\prime}-\mathrm{H}\right), 6.77$ (s, $\left.1 \mathrm{H}, 2-\mathrm{H}\right), 6.88$ (d, $J=$ $\left.8.3 \mathrm{~Hz}, 1 \mathrm{H}, 5^{\prime \prime}-\mathrm{H}\right), 7.08\left(\mathrm{~d}, J=1.9 \mathrm{~Hz}, 1 \mathrm{H}, 2^{\prime \prime}-\mathrm{H}\right), 7.15$ (dd, $J=$ 8.3, $\left.1.9 \mathrm{~Hz}, 1 \mathrm{H}, 6^{\prime \prime}-\mathrm{H}\right), 7.31\left(\mathrm{dd}, J=8.3,8.2 \mathrm{~Hz}, 1 \mathrm{H}, 4^{\prime}-\mathrm{H}\right), 7.59$ $(\mathrm{d}, J=15.0 \mathrm{~Hz}, 1 \mathrm{H}, 5-\mathrm{H}), 12.76\left(\mathrm{~s}, 1 \mathrm{H}, 2^{\prime}-\mathrm{OH}\right), 15.01$ (d, $J=$ $1.0 \mathrm{~Hz}, 1 \mathrm{H} \mathrm{3-OH}$ ) ppm. ${ }^{13} \mathrm{C} \mathrm{NMR}: \delta=55.85,55.91$ and 56.0 $\left(6^{\prime}, 3^{\prime \prime}, 4^{\prime \prime}-\mathrm{OCH}_{3}\right), 101.6\left(\mathrm{C}-5^{\prime}\right), 103.3(\mathrm{C}-2), 109.7\left(\mathrm{C}-2^{\prime \prime}\right), 110.6$ $\left(\mathrm{C}-1^{\prime}\right), 111.1\left(\mathrm{C}-3^{\prime}, \mathrm{C}-5^{\prime \prime}\right), 120.6(\mathrm{C}-4), 122.4\left(\mathrm{C}-6^{\prime \prime}\right), 128.2\left(\mathrm{C}-1^{\prime \prime}\right)$, $134.9\left(\mathrm{C}-4^{\prime}\right), 139.4(\mathrm{C}-5), 149.2\left(\mathrm{C}-3^{\prime \prime}\right), 150.9\left(\mathrm{C}-4^{\prime \prime}\right), 160.2\left(\mathrm{C}-6^{\prime}\right)$, $164.0\left(\mathrm{C}-2^{\prime}\right), 175.0(\mathrm{C}-3), 194.7$ (C-1) ppm. ESI-MS: $m / z(\%)=357$ $[\mathrm{M}+\mathrm{H}]^{+}(16), 379[\mathrm{M}+\mathrm{Na}]^{+}(21) \cdot \mathrm{C}_{20} \mathrm{H}_{20} \mathrm{O}_{6}$ (356.37): calcd. for C 67.41, H 5.66; found C 67.29, H. 5.71.

3-Hydroxy-1-(2-hydroxy-4,6-dimethoxyphenyl)-5-phenylpenta-2,4dien-1-one (11h): Yield 1.30 g (80\%); m.p. $127-128{ }^{\circ} \mathrm{C} .{ }^{1} \mathrm{H}$ NMR: $\delta$ $=3.81\left(\mathrm{~s}, 3 \mathrm{H}, 4^{\prime}-\mathrm{OCH}_{3}\right), 3.89\left(\mathrm{~s}, 3 \mathrm{H}, 6^{\prime}-\mathrm{OCH}_{3}\right), 5.95(\mathrm{~d}, J=$ $\left.2.4 \mathrm{~Hz}, 1 \mathrm{H}, 5^{\prime}-\mathrm{H}\right), 6.08\left(\mathrm{~d}, J=2.4 \mathrm{~Hz}, 1 \mathrm{H}, 3^{\prime}-\mathrm{H}\right), 6.57$ (dd, $J=$ 15.9, $1.2 \mathrm{~Hz}, 1 \mathrm{H}, 4-\mathrm{H}), 6.75(\mathrm{~s}, 1 \mathrm{H}, 2-\mathrm{H}), 7.34-7.42(\mathrm{~m}, 3 \mathrm{H}$, $\left.3^{\prime \prime}, 4^{\prime \prime}, 5^{\prime \prime}-\mathrm{H}\right), 7.53-7.56\left(\mathrm{~m}, 2 \mathrm{H}, 2^{\prime \prime}, 6^{\prime \prime}-\mathrm{H}\right), 7.57(\mathrm{~d}, J=15.9 \mathrm{~Hz}$, $1 \mathrm{H}, 5-\mathrm{H}), 13.62$ (s, $\left.1 \mathrm{H}, 2^{\prime}-\mathrm{OH}\right), 14.73$ (d, $J=1.2 \mathrm{~Hz}, 1 \mathrm{H}, 3-$ $\mathrm{O} H)$ ppm. ${ }^{13} \mathrm{C}$ NMR: $\delta=55.5\left(4^{\prime}-\mathrm{OCH}_{3}\right), 55.6\left(6^{\prime}-\mathrm{OCH}_{3}\right), 91.3$ (C-5'), 94.0 (C-3'), 103.0 (C-2), 104.6 (C-1'), 123.2 (C-4), 127.8 (C$\left.2^{\prime \prime}, 6^{\prime \prime}\right), 128.8\left(\mathrm{C}-3^{\prime \prime}, 5^{\prime \prime}\right), 129.6\left(\mathrm{C}-4^{\prime \prime}\right), 135.3\left(\mathrm{C}-1^{\prime \prime}\right), 138.3(\mathrm{C}-5)$, $161.8\left(\mathrm{C}-6^{\prime}\right), 165.5\left(\mathrm{C}-4^{\prime}\right), 167.2\left(\mathrm{C}-2^{\prime}\right), 173.1$ (C-3), 193.9 (C1) ppm. ESI-MS: $m / z(\%)=326[\mathrm{M}+\mathrm{H}]^{+}(21), 349[\mathrm{M}+\mathrm{Na}]^{+}$ (21), $675[2 \mathrm{M}+\mathrm{Na}]^{+}(6) \cdot \mathrm{C}_{19} \mathrm{H}_{18} \mathrm{O}_{5}$ (326.34): calcd. for $\mathrm{C}$ 69.93, $\mathrm{H}$ 5.56; found C 70.33, H 5.55.

5-(3,4-Dimethoxyphenyl)-3-hydroxy-1-(2-hydroxy-4,6-dimethoxyphenyl)penta-2,4-dien-1-one (11i): Yield 1.49 g (77\%); m.p. 148$150{ }^{\circ} \mathrm{C} .{ }^{1} \mathrm{H}$ NMR: $\delta=3.929,3.932$ and $3.95\left(3 \times \mathrm{s}, 9 \mathrm{H}, 6^{\prime}, 3^{\prime \prime}, 4^{\prime \prime}{ }^{\prime}-\right.$ $\left.\mathrm{OCH}_{3}\right), 6.41\left(\mathrm{~d}, J=8.2 \mathrm{~Hz}, 1 \mathrm{H}, 5^{\prime}-\mathrm{H}\right), 6.45(\mathrm{~d}, J=15.0 \mathrm{~Hz}, 1 \mathrm{H}$, 4-H), 6.59 (dd, $\left.J=8.2,0.8 \mathrm{~Hz}, 1 \mathrm{H}, 3^{\prime}{ }^{\prime}-\mathrm{H}\right), 6.77$ (s, $\left.1 \mathrm{H}, 2-\mathrm{H}\right), 6.88$ $\left(\mathrm{d}, J=8.3 \mathrm{~Hz}, 1 \mathrm{H}, 5^{\prime \prime}-\mathrm{H}\right), 7.08\left(\mathrm{~d}, J=1.9 \mathrm{~Hz}, 1 \mathrm{H}, 2^{\prime \prime}-\mathrm{H}\right), 7.15$ (dd, $\left.J=8.3,1.9 \mathrm{~Hz}, 1 \mathrm{H}, 6^{\prime \prime}-\mathrm{H}\right), 7.31$ (t, $\left.J=8.2 \mathrm{~Hz}, 1 \mathrm{H}, 4^{\prime}-\mathrm{H}\right)$, 7.59 (d, $J=15.0 \mathrm{~Hz}, 1 \mathrm{H}, 5-\mathrm{H}), 12.76$ (s, $\left.1 \mathrm{H}, 2^{\prime}-\mathrm{OH}\right), 15.01$ (d, $J$ $=1.0 \mathrm{~Hz}, 1 \mathrm{H}, 3-\mathrm{OH}) \mathrm{ppm} .{ }^{13} \mathrm{C} \mathrm{NMR}: \delta=55.5\left(4^{\prime}-\mathrm{OCH}_{3}\right), 55.6$, 55.8 and $55.9\left(6^{\prime}, 3^{\prime \prime}, 4^{\prime \prime}-\mathrm{OCH}_{3}\right), 91.3\left(\mathrm{C}-5^{\prime}\right), 94.0\left(\mathrm{C}-3^{\prime}\right), 102.5(\mathrm{C}-$ 2), $104.6\left(\mathrm{C}-1^{\prime}\right), 109.6\left(\mathrm{C}-2^{\prime \prime}\right), 111.0\left(\mathrm{C}-5^{\prime \prime}\right), 120.9$ (C-4), 122.2 (C$\left.6^{\prime \prime}\right), 128.3\left(\mathrm{C}-1^{\prime \prime}\right), 138.5(\mathrm{C}-5), 149.1\left(\mathrm{C}-3^{\prime \prime}\right), 150.6\left(\mathrm{C}-4^{\prime \prime}\right), 161.8$ (C-6'), $165.4\left(\mathrm{C}-4^{\prime}\right), 167.1$ (C-2'), 173.7 (C-3), 193.6 (C-1) ppm. ESI-MS: $m / z(\%)=387[\mathrm{M}+\mathrm{H}]^{+}(29), 409[\mathrm{M}+\mathrm{Na}]^{+}(38), 795$ $[2 \mathrm{M}+\mathrm{Na}]^{+}$(11). $\mathrm{C}_{21} \mathrm{H}_{22} \mathrm{O}_{7}$ (386.40): calcd. for $\mathrm{C}$ 65.28, $\mathrm{H}$ 5.74; found $\mathrm{C} 65.56, \mathrm{H} 5.83$.

Synthesis of the 3-Bromo-2-styryl-4H-chromen-4-ones 3a and 3d-k: Phenyltrimethylammonium tribromide (PTT, $2.1 \mathrm{~g}, 5.5 \mathrm{mmol}$ ) was added to a THF $(60 \mathrm{~mL})$ solution of the appropriate 5-aryl-3-hydroxy-1-(2-hydroxyphenyl)penta-2,4-dien-1-one (11a-i, 5 mmol). The reaction mixture was stirred and allowed to stand at room temperature for $12 \mathrm{~h}$. After that period, the solution was poured into ice $(50 \mathrm{~g})$ and water $(80 \mathrm{~mL})$ and stirred for $20 \mathrm{~min}$, and the obtained yellow solid was removed by filtration. The residue was taken up in chloroform $(50 \mathrm{~mL})$ and washed with water $(3 \times 50 \mathrm{~mL})$. The solvent was removed to dryness and the residue was purified by silica gel column chromatography with dichloromethane as eluent. The obtained residue was recrystallised from ethanol to give the 3-bromo-2-styryl-4H-chromen-4-one (3a or $\mathbf{3 d}-$ k). 
(E)-3-Bromo-2-[2-(4-methoxyphenyl)vinyl]-4H-chromen-4-one (3d): Yield 0.95 g (53\%); m.p. $149-151{ }^{\circ} \mathrm{C} .{ }^{1} \mathrm{H}$ NMR: $\delta=3.86(\mathrm{~s}, 3 \mathrm{H}$, $\left.4^{\prime}-\mathrm{OCH}_{3}\right), 6.94\left(\mathrm{~d}, J=8.8 \mathrm{~Hz}, 2 \mathrm{H}, 3^{\prime}, 5^{\prime}-\mathrm{H}\right), 7.33$ (d, $J=15.9 \mathrm{~Hz}$, $1 \mathrm{H}, \alpha-\mathrm{H}), 7.40$ (ddd, $J=8.5,8.4,0.7 \mathrm{~Hz}, 1 \mathrm{H}, 6-\mathrm{H}), 7.52$ (d, $J=$ $8.0 \mathrm{~Hz}, 1 \mathrm{H}, 8-\mathrm{H}), 7.59$ (d, $\left.J=8.8 \mathrm{~Hz}, 2 \mathrm{H}, 2^{\prime}, 6^{\prime}-\mathrm{H}\right), 7.66(\mathrm{~d}, J=$ $15.9 \mathrm{~Hz}, 1 \mathrm{H}, \beta-\mathrm{H}), 7.69$ (ddd, $J=8.4,8.0,1.6 \mathrm{~Hz}, 1 \mathrm{H}, 7-\mathrm{H}), 8.22$ $(\mathrm{dd}, J=8.5,1.6 \mathrm{~Hz}, 1 \mathrm{H}, 5-\mathrm{H}) \mathrm{ppm} .{ }^{13} \mathrm{C} \mathrm{NMR}: \delta=55.4\left(4^{\prime}-\right.$ $\left.\mathrm{OCH}_{3}\right), 108.9(\mathrm{C}-3), 114.5\left(\mathrm{C}-3^{\prime}, 5^{\prime}\right), 116.7(\mathrm{C}-\alpha), 117.4(\mathrm{C}-8), 122.0$ (C-10), 125.2 (C-6), 126.3 (C-5), $127.6\left(\mathrm{C}-1^{\prime}\right), 129.8\left(\mathrm{C}-2^{\prime}, 6^{\prime}\right), 133.9$ (C-7), 139.3 (C- $\beta$ ), 154.8 (C-9), 158.8 (C-2), 161.4 (C-4'), 172.7 (C4) $\mathrm{ppm}$. ESI-MS: $m / z(\%)=357[\mathrm{M}+\mathrm{H}]^{+}\left({ }^{79} \mathrm{Br}, 65\right), 359[\mathrm{M}+\mathrm{H}]$ ${ }^{+}\left({ }^{81} \mathrm{Br}, 66\right), 379[\mathrm{M}+\mathrm{Na}]^{+}\left({ }^{79} \mathrm{Br}, 29\right), 381[\mathrm{M}+\mathrm{Na}]^{+}\left({ }^{81} \mathrm{Br}, 89\right)$, $735[2 \mathrm{M}+\mathrm{Na}]^{+}\left({ }^{79} \mathrm{Br}, 19\right), 737[2 \mathrm{M}+\mathrm{Na}]^{+}\left({ }^{81} \mathrm{Br}, 40\right) \cdot \mathrm{C}_{18} \mathrm{H}_{13} \mathrm{BrO}_{3}$ (357.20): calcd. for C 60.52, H 3.67; found C 60.14, H 3.66.

(E)-3-Bromo-2-[2-(3,4-dimethoxyphenyl)vinyl]-4H-chromen-4-one (3e): This compound showed spectroscopic and analytical data identical to those previously reported. ${ }^{[27]}$

(E)-3-Bromo-7-methoxy-2-(2-phenylvinyl)-4H-chromen-4-one (3f): Yield 1.73 g (97\%); m.p. $174-176{ }^{\circ} \mathrm{C} .{ }^{1} \mathrm{H}$ NMR: $\delta=3.95(\mathrm{~s}, 3 \mathrm{H}$, $\left.7-\mathrm{OCH}_{3}\right), 6.93(\mathrm{~d}, J=2.4 \mathrm{~Hz}, 1 \mathrm{H}, 8-\mathrm{H}), 6.97$ (dd, $J=8.8,2.4 \mathrm{~Hz}$, $1 \mathrm{H}, 6-\mathrm{H}), 7.40-7.45\left(\mathrm{~m}, 3 \mathrm{H}, 3^{\prime}, 4^{\prime}, 5^{\prime}-\mathrm{H}\right), 7.46(\mathrm{~d}, J=16.0 \mathrm{~Hz}, 1$ $\mathrm{H}, \alpha-\mathrm{H}), 7.63-7.66\left(\mathrm{~m}, 2 \mathrm{H}, 2^{\prime}, 6^{\prime}-\mathrm{H}\right), 7.67$ (d, $J=16.0 \mathrm{~Hz}, 1 \mathrm{H}, \beta-$ $\mathrm{H}), 8.13(\mathrm{~d}, J=8.8 \mathrm{~Hz}, 1 \mathrm{H}, 5-\mathrm{H}) \mathrm{ppm} .{ }^{13} \mathrm{C} \mathrm{NMR}: \delta=55.9(7-$ $\left.\mathrm{OCH}_{3}\right), 99.7$ (C-8), 109.9 (C-3), 114.7 (C-6), 115.9 (C-10), 119.3 $(\mathrm{C}-\alpha), 127.7$ (C-5), $128.0\left(\mathrm{C}-2^{\prime}, 6^{\prime}\right), 129.0\left(\mathrm{C}-3^{\prime}, 5^{\prime}\right), 130.2\left(\mathrm{C}-4^{\prime}\right)$, $135.0\left(\mathrm{C}-1^{\prime}\right), 138.9$ (C- $\left.\beta\right), 156.6$ (C-9), 157.9 (C-2), 164.4 (C-7), 172.1 (C-4) ppm. ESI-MS: $m / z(\%)=357[\mathrm{M}+\mathrm{H}]^{+}\left({ }^{79} \mathrm{Br}, 97\right), 359$ $[\mathrm{M}+\mathrm{H}]^{+}\left({ }^{81} \mathrm{Br}, 96\right), 379[\mathrm{M}+\mathrm{Na}]^{+}\left({ }^{79} \mathrm{Br}, 39\right), 381[\mathrm{M}+\mathrm{Na}]^{+}$ $\left({ }^{81} \mathrm{Br}, 83\right), 395[\mathrm{M}+\mathrm{K}]^{+}\left({ }^{79} \mathrm{Br}, 10\right), 397[\mathrm{M}+\mathrm{K}]^{+}\left({ }^{81} \mathrm{Br}, 13\right), 735$ $[2 \mathrm{M}+\mathrm{Na}]^{+}\left({ }^{79} \mathrm{Br}, 50\right), 737[2 \mathrm{M}+\mathrm{Na}]^{+}\left({ }^{81} \mathrm{Br}, 98\right) \cdot \mathrm{C}_{18} \mathrm{H}_{13} \mathrm{BrO}_{3}$ (357.20): calcd. for C 60.52, H 3.67; found C 60.43, H 4.12.

(E)-3-Bromo-2-[2-(3,4-dimethoxyphenyl)vinyl]-7-methoxy-4Hchromen-4-one (3g): Yield 1.25 g $(60 \%)$; m.p. $195-917{ }^{\circ} \mathrm{C} .{ }^{1} \mathrm{H}$ NMR: $\delta=3.937$ and $3.943\left(2 \times \mathrm{s}, 6 \mathrm{H}, 7,4^{\prime}-\mathrm{OCH}_{3}\right), 3.97(\mathrm{~s}, 3 \mathrm{H}$, $\left.3^{\prime}-\mathrm{OCH}_{3}\right), 6.90(\mathrm{~d}, J=2.3 \mathrm{~Hz}, 1 \mathrm{H}, 8-\mathrm{H}), 6.91(\mathrm{~d}, J=8.3 \mathrm{~Hz}, 1$ $\left.\mathrm{H}, 5^{\prime}-\mathrm{H}\right), 6.95$ (dd, $\left.J=8.8,2.3 \mathrm{~Hz}, 1 \mathrm{H}, 6-\mathrm{H}\right), 7.12(\mathrm{~d}, J=1.9 \mathrm{~Hz}$, $\left.1 \mathrm{H}, 2^{\prime}-\mathrm{H}\right), 7.21\left(\mathrm{dd}, J=8.3,1.9 \mathrm{~Hz}, 1 \mathrm{H}, 6^{\prime}-\mathrm{H}\right), 7.28$ (d, $J=$ $15.9 \mathrm{~Hz}, 1 \mathrm{H}, \alpha-\mathrm{H}), 7.60(\mathrm{~d}, J=15.9 \mathrm{~Hz}, 1 \mathrm{H}, \beta-\mathrm{H}), 8.11(\mathrm{~d}, J=$ $8.8 \mathrm{~Hz}, 1 \mathrm{H}, 5-\mathrm{H}) \mathrm{ppm} .{ }^{13} \mathrm{C} \mathrm{NMR}: \delta=55.86,55.92$ and 56.0 (7,3', $\left.4^{\prime}-\mathrm{OCH}_{3}\right), 99.7$ (C-8), $109.1(\mathrm{C}-3), 109.6\left(\mathrm{C}-2^{\prime}\right), 111.1\left(\mathrm{C}-5^{\prime}\right)$, 114.5 (C-6), 115.9 (C-10), 116.9 (C- $\alpha), 122.4$ (C-6'), 127.7 (C-5), 128.0 (C-1'), 138.9 (C- $\beta$ ), 149.3 (C-3'), 151.1 (C-4'), 156.5 (C-9), 158.3 (C-2), 164.3 (C-7), 172.0 (C-4) ppm. ESI-MS: $m / z(\%)=417$ $[\mathrm{M}+\mathrm{H}]^{+}\left({ }^{79} \mathrm{Br}, 34\right), 419[\mathrm{M}+\mathrm{H}]^{+}\left({ }^{81} \mathrm{Br}, 33\right), 439[\mathrm{M}+\mathrm{Na}]^{+}\left({ }^{79} \mathrm{Br}\right.$, 13), $441[\mathrm{M}+\mathrm{Na}]^{+}\left({ }^{81} \mathrm{Br}, 13\right), 855[2 \mathrm{M}+\mathrm{Na}]^{+}\left({ }^{79} \mathrm{Br}, 13\right), 857[2 \mathrm{M}$ $+\mathrm{Na}^{+}\left({ }^{81} \mathrm{Br}, 25\right) \cdot \mathrm{C}_{20} \mathrm{H}_{17} \mathrm{BrO}_{5}$ (417.25): calcd. for $\mathrm{C} 57.57, \mathrm{H} 4.11$; found C 57.76, H 4.18 .

(E)-3-Bromo-5-methoxy-2-(2-phenylvinyl)-4H-chromen-4-one (3h): Yield $1.50 \mathrm{~g}(84 \%)$; m.p. $172-173{ }^{\circ} \mathrm{C} .{ }^{1} \mathrm{H}$ NMR: $\delta=3.97$ (s, $3 \mathrm{H}$, $\left.5-\mathrm{OCH}_{3}\right), 6.82(\mathrm{dd}, J=8.4,0.9 \mathrm{~Hz}, 1 \mathrm{H}, 6-\mathrm{H}), 7.10(\mathrm{dd}, J=8.4$, $0.9 \mathrm{~Hz}, 1 \mathrm{H}, 8-\mathrm{H}), 7.42(\mathrm{~d}, J=16.0 \mathrm{~Hz}, 1 \mathrm{H}, \alpha-\mathrm{H}), 7.40-7.45(\mathrm{~m}$, $\left.3 \mathrm{H}, 3^{\prime}, 4^{\prime}, 5^{\prime}-\mathrm{H}\right), 7.58(\mathrm{t}, J=8.4 \mathrm{~Hz}, 1 \mathrm{H}, 7-\mathrm{H}), 7.63(\mathrm{~d}, J=16.0 \mathrm{~Hz}$, $1 \mathrm{H}, \beta-\mathrm{H}), 7.62-7.64\left(\mathrm{~m}, 2 \mathrm{H}, 2^{\prime}, 6^{\prime}-\mathrm{H}\right) \mathrm{ppm} .{ }^{13} \mathrm{C} \mathrm{NMR}: \delta=56.4$ $\left(5-\mathrm{OCH}_{3}\right), 106.5$ (C-6), 109.4 (C-8), 111.4 (C-3), 112.7 (C-10), 119.1 $(\mathrm{C}-\alpha), 127.9\left(\mathrm{C}-2^{\prime}, 6^{\prime}\right), 129.0\left(\mathrm{C}-3^{\prime}, 5^{\prime}\right), 130.1\left(\mathrm{C}-4^{\prime}\right), 134.1(\mathrm{C}-7)$, 134.9 (C-1'), 138.8 (C- $\beta$ ), 156.5 (C-9), 156.8 (C-2), 159.7 (C-5), 171.7 (C-4) ppm. ESI-MS: $m / z(\%)=357[\mathrm{M}+\mathrm{H}]^{+}\left({ }^{79} \mathrm{Br}, 83\right), 359$ $[\mathrm{M}+\mathrm{H}]^{+}\left({ }^{81} \mathrm{Br}, 80\right), 379[\mathrm{M}+\mathrm{Na}]^{+}\left({ }^{79} \mathrm{Br}, 28\right), 381[\mathrm{M}+\mathrm{Na}]^{+}$ $\left({ }^{81} \mathrm{Br}, 28\right), 735[2 \mathrm{M}+\mathrm{Na}]^{+}\left({ }^{79} \mathrm{Br}, 51\right), 737[2 \mathrm{M}+\mathrm{Na}]^{+}\left({ }^{81} \mathrm{Br}, 100\right)$, $1091[3 \mathrm{M}+\mathrm{Na}]^{+}\left({ }^{79} \mathrm{Br}, 4\right), 1093[3 \mathrm{M}+\mathrm{Na}]^{+}\left({ }^{81} \mathrm{Br}, 11\right)$. $\mathrm{C}_{18} \mathrm{H}_{13} \mathrm{BrO}_{3}$ (357.20): calcd. for $\mathrm{C} 60.52, \mathrm{H} \mathrm{3.67}$; found $\mathrm{C} 60.77, \mathrm{H}$ 3.69 .
(E)-3-Bromo-2-[2-(3,4-dimethoxyphenyl)vinyl]-5-methoxy-4Hchromen-4-one (3i): Yield 1.21 g (58\%); m.p. $190-192{ }^{\circ} \mathrm{C} .{ }^{1} \mathrm{H}$ NMR: $\delta=3.94\left(\mathrm{~s}, 3 \mathrm{H}, 4^{\prime}-\mathrm{OCH}_{3}\right), 3.977\left(\mathrm{~s}, 3 \mathrm{H}, 3^{\prime}-\mathrm{OCH}_{3}\right), 3.982(\mathrm{~s}, 3 \mathrm{H}$, $\left.5-\mathrm{OCH}_{3}\right), 6.82(\mathrm{~d}, J=8.2 \mathrm{~Hz}, 1 \mathrm{H}, 6-\mathrm{H}), 6.91(\mathrm{~d}, J=8.3 \mathrm{~Hz}, 1 \mathrm{H}$, $\left.5^{\prime}-\mathrm{H}\right), 7.10(\mathrm{~d}, J=8.4 \mathrm{~Hz}, 1 \mathrm{H}, 8-\mathrm{H}), 7.13\left(\mathrm{~d}, J=1.7 \mathrm{~Hz}, 1 \mathrm{H}, 2^{\prime}-\right.$ H), $7.22\left(\mathrm{dd}, J=8.3,1.7 \mathrm{~Hz}, 1 \mathrm{H}, 6^{\prime}-\mathrm{H}\right), 7.27(\mathrm{~d}, J=15.8 \mathrm{~Hz}, 1 \mathrm{H}$, $\alpha-\mathrm{H}), 7.576(\mathrm{dd}, J=8.4,8.2 \mathrm{~Hz}, 1 \mathrm{H}, 7-\mathrm{H}), 7.578$ (d, $J=15.8 \mathrm{~Hz}, 1$ $\mathrm{H}, \beta-\mathrm{H}) \mathrm{ppm} .{ }^{13} \mathrm{C}$ NMR: $\delta=56.0\left(4^{\prime}-\mathrm{OCH}_{3}\right), 56.3$ and $56.5\left(5,3^{\prime}-\right.$ $\left.\mathrm{OCH}_{3}\right), 106.5$ (C-6), 109.4 (C-8), $109.6\left(\mathrm{C}-2^{\prime}\right), 110.6$ (C-3), 111.1 (C-5'), 112.8 (C-10), 116.9 (C- $\alpha), 122.4\left(\mathrm{C}-6^{\prime}\right), 128.1$ (C-1'), 133.9 (C-7), 138.8 (C- $\beta$ ), 149.3 (C-3'), 151.0 (C-4'), 156.8 (C-9), 156.9 (C2), 159.7 (C-5), $171.8(\mathrm{C}-4)$ ppm. ESI-MS: $m / z(\%)=417[\mathrm{M}+$ $\mathrm{H}^{+}\left({ }^{79} \mathrm{Br}, 26\right), 419[\mathrm{M}+\mathrm{H}]^{+}\left({ }^{81} \mathrm{Br}, 25\right), 439[\mathrm{M}+\mathrm{Na}]^{+}\left({ }^{79} \mathrm{Br}, 13\right)$, $441[\mathrm{M}+\mathrm{Na}]^{+}\left({ }^{81} \mathrm{Br}, 13\right), 855[2 \mathrm{M}+\mathrm{Na}]^{+}\left({ }^{79} \mathrm{Br}, 15\right), 857[2 \mathrm{M}+$ $\mathrm{Na}]^{+}\left({ }^{81} \mathrm{Br}, 30\right) . \mathrm{C}_{20} \mathrm{H}_{17} \mathrm{BrO}_{5}$ (417.25): calcd. for $\mathrm{C} 57.57, \mathrm{H} 4.11$; found $\mathrm{C} 57.27, \mathrm{H} 4.21$.

(E)-3-Bromo-5,7-dimethoxy-2-(2-phenylvinyl)-4H-chromen-4-one (3j): Yield $0.95 \mathrm{~g}(49 \%)$; m.p. $181-183{ }^{\circ} \mathrm{C} .{ }^{1} \mathrm{H}$ NMR: $\delta=3.928$ and $3.931\left(2 \times \mathrm{s}, 6 \mathrm{H}, 5,7-\mathrm{OCH}_{3}\right), 6.36(\mathrm{~d}, J=2.3 \mathrm{~Hz}, 1 \mathrm{H}, 6-\mathrm{H}), 6.53$ $(\mathrm{d}, J=2.3 \mathrm{~Hz}, 1 \mathrm{H}, 8-\mathrm{H}), 7.40(\mathrm{~d}, J=16.0 \mathrm{~Hz}, 1 \mathrm{H}, \alpha-\mathrm{H}), 7.41-$ 7.44 (m, $\left.3 \mathrm{H}, 3^{\prime}, 4^{\prime}, 5^{\prime}-\mathrm{H}\right), 7.59$ (d, $\left.J=16.0 \mathrm{~Hz}, 1 \mathrm{H}, \beta-\mathrm{H}\right), 7.61-$ $7.64\left(\mathrm{~m}, 2 \mathrm{H}, 2^{\prime}, 6^{\prime}-\mathrm{H}\right) \mathrm{ppm} .{ }^{13} \mathrm{C} \mathrm{NMR}: \delta=55.8$ and $56.4(5,7-$ $\left.\mathrm{OCH}_{3}\right), 92.2(\mathrm{C}-8), 96.3(\mathrm{C}-6), 107.6(\mathrm{C}-10), 111.6(\mathrm{C}-3), 119.2(\mathrm{C}-$ a), $127.9\left(\mathrm{C}-2^{\prime}, 6^{\prime}\right), 129.0\left(\mathrm{C}-3^{\prime}, 5^{\prime}\right), 130.0\left(\mathrm{C}-4^{\prime}\right), 135.1\left(\mathrm{C}-1^{\prime}\right), 138.2$ (C- $\beta), 156.1$ (C-2), 158.4 (C-9), 160.9 (C-5), 164.3 (C-7), 171.0 (C4) ppm. ESI-MS: $m / z=387[\mathrm{M}+\mathrm{H}]^{+}\left({ }^{79} \mathrm{Br}, 33\right), 389[\mathrm{M}+\mathrm{H}]^{+}$ $\left({ }^{81} \mathrm{Br}, 33\right), 409[\mathrm{M}+\mathrm{Na}]^{+}\left({ }^{79} \mathrm{Br}, 8\right), 411[\mathrm{M}+\mathrm{Na}]^{+}\left({ }^{81} \mathrm{Br}, 8\right), 795$ $[2 \mathrm{M}+\mathrm{Na}]^{+}\left({ }^{79} \mathrm{Br}, 18\right), 797[2 \mathrm{M}+\mathrm{Na}]^{+}\left({ }^{81} \mathrm{Br}, 35\right) \cdot \mathrm{C}_{19} \mathrm{H}_{15} \mathrm{BrO}_{4}$ (387.22): calcd. for C 58.93, H 3.90; found C 58.77, H 4.07.

(E)-3-Bromo-2-[2-(3,4-dimethoxyphenyl)vinyl]-5,7-dimethoxy-4Hchromen-4-one (3k): Yield $0.67 \mathrm{~g}(30 \%)$; m.p. $208-210{ }^{\circ} \mathrm{C} .{ }^{1} \mathrm{H}$ NMR: $\delta=3.91\left(\mathrm{~s}, 3 \mathrm{H}, 5-\mathrm{OCH}_{3}\right), 3.92\left(\mathrm{~s}, 3 \mathrm{H}, 7-\mathrm{OCH}_{3}\right), 3.94(\mathrm{~s}$, $\left.3 \mathrm{H}, 4^{\prime}-\mathrm{OCH}_{3}\right), 3.96\left(\mathrm{~s}, 3 \mathrm{H}, 3^{\prime}-\mathrm{OCH}_{3}\right), 6.33(\mathrm{~d}, J=2.3 \mathrm{~Hz}, 1 \mathrm{H}$, 6-H), $6.50(\mathrm{~d}, J=2.3 \mathrm{~Hz}, 1 \mathrm{H}, 8-\mathrm{H}), 6.90$ (d, $J=8.5 \mathrm{~Hz}, 1 \mathrm{H}, 5^{\prime}-$ $\mathrm{H}), 7.10\left(\mathrm{~d}, J=1.9 \mathrm{~Hz}, 1 \mathrm{H}, 2^{\prime}-\mathrm{H}\right), 7.19(\mathrm{dd}, J=8.5,1.9 \mathrm{~Hz}, 1 \mathrm{H}$, $\left.6^{\prime}-\mathrm{H}\right), 7.20(\mathrm{~d}, J=15.9 \mathrm{~Hz}, 1 \mathrm{H}, \alpha-\mathrm{H}), 7.50(\mathrm{~d}, J=15.9 \mathrm{~Hz}, 1 \mathrm{H}$, $\beta-\mathrm{H}) \mathrm{ppm} .{ }^{13} \mathrm{C} \mathrm{NMR}: \delta=55.8\left(7-\mathrm{OCH}_{3}\right), 55.9\left(3^{\prime}-\mathrm{OCH}_{3}\right), 56.0\left(4^{\prime}-\right.$ $\left.\mathrm{OCH}_{3}\right), 56.3\left(5-\mathrm{OCH}_{3}\right), 92.1(\mathrm{C}-8), 96.2(\mathrm{C}-6), 107.5(\mathrm{C}-10), 109.6$ (C-2'), 110.8 (C-3), 111.1 (C-5'), 116.9 (C- $\alpha), 122.2$ (C-6'), 128.1 $\left(\mathrm{C}-1^{\prime}\right), 138.1$ (C- $\left.\beta\right), 149.2\left(\mathrm{C}^{\prime} 3^{\prime}\right), 150.9\left(\mathrm{C}-4^{\prime}\right), 156.4(\mathrm{C}-2), 158.4$ (C-9), 160.8 (C-5), 164.2 (C-7), 170.9 (C-4) ppm. ESI-MS: m/z (\%) $=447[\mathrm{M}+\mathrm{H}]^{+}\left({ }^{79} \mathrm{Br}, 46\right), 449[\mathrm{M}+\mathrm{H}]^{+}\left({ }^{81} \mathrm{Br}, 21\right), 469[\mathrm{M}+$ $\mathrm{Na}]^{+}\left({ }^{79} \mathrm{Br}, 16\right), 471[\mathrm{M}+\mathrm{Na}]^{+}\left({ }^{81} \mathrm{Br}, 16\right), 915[2 \mathrm{M}+\mathrm{Na}]^{+}\left({ }^{79} \mathrm{Br}\right.$, 19), $917[2 \mathrm{M}+\mathrm{Na}]^{+}\left({ }^{81} \mathrm{Br}, 38\right) \cdot \mathrm{C}_{21} \mathrm{H}_{19} \mathrm{BrO}_{6}$ (447.28): calcd. for $\mathrm{C}$ 56.39, H 4.28; found C 56.27, H 4.36.

Synthesis of the Methoxylated 2,3-Diaryl-9H-xanthen-9-ones 5g-s: The appropriate styrene $(\mathbf{4 a}-\mathbf{c}, 1.6 \mathrm{mmol}$ for styrene $\mathbf{4 a}$ and $0.8 \mathrm{mmol}$ for styrenes $\mathbf{4 b}$ and $\mathbf{4 c}$ ) was added to a mixture of the appropriate 3-bromo-2-styryl-4H-chromen-4-one (3d-k, $0.4 \mathrm{mmol})$, triphenylphosphane $(10.5 \mathrm{mg}, 0.04 \mathrm{mmol})$, tetrakis(triphenylphosphane)palladium $(0)(23.1 \mathrm{mg}, 0.02 \mathrm{mmol})$ and triethylamine $(55.8 \mu \mathrm{L}, 0.4 \mathrm{mmol})$ in $N$-methyl-2-pyrrolidin-1-one $(6 \mathrm{~mL})$. The mixture was stirred under different time and temperature conditions according to the substitution of the compounds (Table 3 ). The mixture was then poured into water $(20 \mathrm{~mL})$ and ice $(10 \mathrm{~g})$ and extracted with diethyl ether $(4 \times 25 \mathrm{~mL})$ and dried with anhydrous sodium sulfate. The solvent was removed to dryness and the residue was taken up in dichloromethane $(5 \mathrm{~mL})$ and purified by thin layer chromatography (eluent: dichloromethane/light petroleum 7:3). The major spot with higher $R_{\mathrm{f}}$ value corresponds to the methoxylated 2,3-diaryl-9H-xanthen-9-one (5g-s) and the minor spot with lower $R_{\mathrm{f}}$ corresponds to the 2,3-diaryl-3,4-dihydro- $9 H$ - 
xanthen-9-one $(\mathbf{6 g}-\mathbf{s})$. In the cases of the $(E)$-3-bromo-5-methoxy2-styryl-4H-chromen-4-ones $\mathbf{5 n}-\mathbf{s}$, it was also possible to isolate the 6,7-diaryl-1-hydroxy-9H-xanthen-9-ones $\mathbf{1 2 n}-\mathbf{s}$. The obtained methoxylated 2,3-diaryl-9H-xanthen-9-ones were recrystallised from ethanol and obtained in moderate to good yields (Table 3).

3-(4-Methoxyphenyl)-2-phenyl-9H-xanthen-9-one (5g): M.p. 150$153{ }^{\circ} \mathrm{C} .{ }^{1} \mathrm{H}$ NMR: $\delta=3.80\left(\mathrm{~s}, 3 \mathrm{H}, 4^{\prime \prime}{ }^{\prime}-\mathrm{OCH}_{3}\right), 6.80(\mathrm{~d}, J=8.8 \mathrm{~Hz}$, $\left.2 \mathrm{H}, 3^{\prime \prime}, 5^{\prime \prime}-\mathrm{H}\right), 7.13\left(\mathrm{~d}, J=8.8 \mathrm{~Hz}, 2 \mathrm{H}, 2^{\prime \prime}, 6^{\prime \prime}-\mathrm{H}\right), 7.17-7.20(\mathrm{~m}$, $\left.2 \mathrm{H}, 2^{\prime}, 6^{\prime}-\mathrm{H}\right), 7.23-7.27$ (m, $\left.3 \mathrm{H}, 3^{\prime}, 4^{\prime}, 5^{\prime}-\mathrm{H}\right), 7.39$ (ddd, $J=7.7$, 7.6, $0.9 \mathrm{~Hz}, 1 \mathrm{H}, 7-\mathrm{H}), 7.51(\mathrm{~d}, J=8.5 \mathrm{~Hz}, 1 \mathrm{H}, 5-\mathrm{H}), 7.53(\mathrm{~s}, 1 \mathrm{H}$, 4-H), 7.73 (ddd, $J=8.5,7.6,1.7 \mathrm{~Hz}, 1 \mathrm{H}, 6-\mathrm{H}), 8.34$ (s, $1 \mathrm{H}, 1-\mathrm{H})$, $8.37(\mathrm{dd}, J=7.7,1.7 \mathrm{~Hz}, 1 \mathrm{H}, 8-\mathrm{H}) \mathrm{ppm} .{ }^{13} \mathrm{C}$ NMR: $\delta=55.2\left(4^{\prime \prime}-\right.$

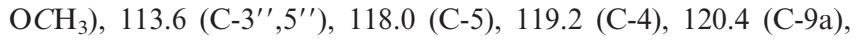
122.0 (C-8a), 123.9 (C-7), 126.7 (C-8), 126.8 (C-4'), 128.1 (C-3', $\left.5^{\prime}\right)$, $128.4(\mathrm{C}-1), 129.9\left(\mathrm{C}-2^{\prime}, 6^{\prime}\right), 130.8\left(\mathrm{C}-2^{\prime \prime}, 6^{\prime \prime}\right), 132.1\left(\mathrm{C}-1^{\prime \prime}\right), 134.7$ (C-6), 137.0 (C-2), 140.1 (C-1'), 147.3 (C-3), 155.3 (C-4a), 156.3 (C-4b), 159.1 (C-4' ), 177.0 (C-9) ppm. EI-MS: $m / z(\%)=378$ $[\mathrm{M}]^{+\cdot}$ (100), 377 (23), 363 (10), 347 (23), 335 (11), 334 (13), 305 (10), 276 (6). $\mathrm{C}_{26} \mathrm{H}_{18} \mathrm{O}_{3}$ (378.42): calcd. for $\mathrm{C} 82.52, \mathrm{H} \mathrm{4.79}$; found C 82.24, H 5.03.

3-(4-Methoxyphenyl)-2-phenyl-3,4-dihydro-9H-xanthen-9-one (6g): Yellowish oil. ${ }^{1} \mathrm{H}$ NMR: $\delta=2.96\left(\mathrm{dd}, J=17.3,1.3 \mathrm{~Hz}, 1 \mathrm{H}, 4_{\text {trans }^{-}}{ }^{-}\right.$ $\mathrm{H}), 3.61$ (dd, $\left.J=17.3,8.8 \mathrm{~Hz}, 1 \mathrm{H}, 4_{\text {cis }}-\mathrm{H}\right), 3.70$ (s, $3 \mathrm{H}, 4^{\prime \prime}{ }^{\prime}-\mathrm{OCH}_{3}$ ), $4.25(\mathrm{dd}, J=8.8,1.3 \mathrm{~Hz}, 1 \mathrm{H}, 3-\mathrm{H}), 6.76(\mathrm{~d}, J=8.7 \mathrm{~Hz}, 2 \mathrm{H}$, $\left.3^{\prime \prime}, 5^{\prime \prime}-\mathrm{H}\right), 7.21\left(\mathrm{~d}, J=8.7 \mathrm{~Hz}, 2 \mathrm{H}, 2^{\prime \prime}, 6^{\prime \prime}-\mathrm{H}\right), 7.23-7.29$ (m, $3 \mathrm{H}$, $\left.3^{\prime}, 4^{\prime}, 5^{\prime}-\mathrm{H}\right), 7.33(\mathrm{~d}, J=8.5 \mathrm{~Hz}, 1 \mathrm{H}, 5-\mathrm{H}), 7.36(\mathrm{ddd}, J=8.2,7.9$, $1.0 \mathrm{~Hz}, 1 \mathrm{H}, 7-\mathrm{H}), 7.46-7.49$ (m, $\left.2 \mathrm{H}, 2^{\prime \prime}, 6^{\prime \prime}-\mathrm{H}\right), 7.54$ (s, $1 \mathrm{H}, 1-$ $\mathrm{H}), 7.58$ (ddd, $J=8.5,8.2,1.6 \mathrm{~Hz}, 1 \mathrm{H}, 6-\mathrm{H}), 8.27$ (dd, $J=7.9$, $1.6 \mathrm{~Hz}, 1 \mathrm{H}, 8-\mathrm{H})$ ppm. ${ }^{13} \mathrm{C} \mathrm{NMR}: \delta=36.8(\mathrm{C}-4), 40.8(\mathrm{C}-3), 55.1$ $\left(4^{\prime \prime}-\mathrm{OCH}_{3}\right), 114.2\left(\mathrm{C}-3^{\prime \prime}, 5^{\prime \prime}\right), 116.4(\mathrm{C}-1), 116.8$ (C-9a), $118.0(\mathrm{C}-$ 5), 123.8 (C-8a), 125.0 (C-7), 125.6 (C-2',6'), 126.1 (C-8), 127.5 (C$\left.4^{\prime}\right), 128.3\left(\mathrm{C}-2^{\prime \prime}, 6^{\prime \prime}\right), 128.4\left(\mathrm{C}-3^{\prime}, 5^{\prime}\right), 132.5\left(\mathrm{C}-1^{\prime \prime}\right), 133.0(\mathrm{C}-6)$, 135.6 (C-2), $139.0\left(\mathrm{C}-1^{\prime}\right), 155.8$ (C-4b), $158.6\left(\mathrm{C}-4^{\prime \prime}\right), 162.6$ (C-4a), 174.2 (C-9) ppm. EI-MS: $m / z(\%)=380$ [M] $^{+\cdot}(14), 379(29), 378$ (100), 377 (30), 363 (16), 347 (24), 335 (13), 334 (14), 333 (11), 305 (11), 276 (8), 215 (6), 135 (7), 105 (5). HRMS (EI): calcd. for $\mathrm{C}_{26} \mathrm{H}_{18} \mathrm{O}_{3}[\mathrm{M}]^{+\cdot} 378.1256$; found 378.1244 .

2,3-Bis(4-methoxyphenyl)-9H-xanthen-9-one (5h): M.p. $164-166{ }^{\circ} \mathrm{C}$. ${ }^{1} \mathrm{H}$ NMR: $\delta=3.807$ and $3.813\left(\mathrm{~s}, 6 \mathrm{H}, 4^{\prime}, 4^{\prime \prime}{ }^{\prime}-\mathrm{OCH}_{3}\right), 6.80(\mathrm{~d}, J=$ $\left.8.8 \mathrm{~Hz}, 2 \mathrm{H}, 3^{\prime}, 5^{\prime}-\mathrm{H}\right), 6.82\left(\mathrm{~d}, J=8.8 \mathrm{~Hz}, 2 \mathrm{H}, 3^{\prime \prime}, 5^{\prime \prime}-\mathrm{H}\right), 7.11$ (d, $\left.J=8.8 \mathrm{~Hz}, 2 \mathrm{H}, 2^{\prime}, 6^{\prime}-\mathrm{H}\right), 7.14\left(\mathrm{~d}, J=8.8 \mathrm{~Hz}, 2 \mathrm{H}, 2^{\prime \prime}, 6^{\prime \prime}-\mathrm{H}\right), 7.40$ (ddd, $J=7.8,7.6,1.0 \mathrm{~Hz}, 1 \mathrm{H}, 7-\mathrm{H}), 7.52(\mathrm{~d}, J=7.8 \mathrm{~Hz}, 1 \mathrm{H}, 5-$ H), 7.52 (s, $1 \mathrm{H}, 4-\mathrm{H}), 7.74$ (ddd, $J=7.8,7.6,1.7 \mathrm{~Hz} 1 \mathrm{H}, 6-\mathrm{H})$, 8.32 (s, $1 \mathrm{H}, 1-\mathrm{H}), 8.37$ (dd, $J=7.8,1.7 \mathrm{~Hz}, 1 \mathrm{H}, 8-\mathrm{H}) \mathrm{ppm} .{ }^{13} \mathrm{C}$ NMR: $\delta=55.19$ and $55.21\left(4^{\prime}, 4^{\prime \prime}-\mathrm{OCH}_{3}\right), 113.5$ and $113.6(\mathrm{C}-$ 3',5', C-3"',5'), 118.0 (C-5), 119.2 (C-4), 120.4 (C-9a), 122.0 (C8a), 123.9 (C-7), 126.8 (C-8), 128.1 (C-1), 130.8 and 131.0 (C-2', 6', $\left.\mathrm{C}-2^{\prime \prime}, 6^{\prime \prime}\right), 132.35$ and 132.43 (C-1', C-1' $), 134.7$ (C-6), 136.6 (C2), 147.3 (C-3), 155.1 (C-4a), 156.3 (C-4b), 158.5 (C-4'), 159.1 (C$\left.4^{\prime \prime}\right), 177.0(\mathrm{C}-9)$ ppm. EI-MS: $m / z(\%)=408[\mathrm{M}]^{+\cdot}(100), 407(8)$, 393 (14), 377 (19), 365 (8), 350 (12), 334 (6), 333 (6), 321 (10). $\mathrm{C}_{27} \mathrm{H}_{20} \mathrm{O}_{4}$ (408.45): calcd. for $\mathrm{C} 79.40, \mathrm{H} \mathrm{4.94}$; found $\mathrm{C} 79.12, \mathrm{H}$ 5.03 .

2,3-Bis(4-methoxyphenyl)-3,4-dihydro-9H-xanthen-9-one (6h): Yellowish oil. ${ }^{1} \mathrm{H}$ NMR: $\delta=2.96\left(\mathrm{dd}, J=17.3,1.5 \mathrm{~Hz}, 1 \mathrm{H}, 4_{\text {trans }}-\mathrm{H}\right)$, 3.62 (dd, $\left.J=17.3,8.3 \mathrm{~Hz}, 1 \mathrm{H}, 4_{\text {cis }}-\mathrm{H}\right), 3.72$ (s, $3 \mathrm{H}, 4^{\prime \prime}{ }^{\prime}-\mathrm{OCH}_{3}$ ), 3.79 (s, $\left.3 \mathrm{H}, 4^{\prime}-\mathrm{OCH}_{3}\right), 4.24$ (brd, $\left.J=8.3 \mathrm{~Hz}, 1 \mathrm{H}, 3-\mathrm{H}\right), 6.77$ (d, $\left.J=8.7 \mathrm{~Hz}, 2 \mathrm{H}, 3^{\prime \prime}, 5^{\prime \prime}-\mathrm{H}\right), 6.83\left(\mathrm{~d}, J=8.9 \mathrm{~Hz}, 2 \mathrm{H}, 3^{\prime}, 5^{\prime}-\mathrm{H}\right), 7.21$ (d, $\left.J=8.7 \mathrm{~Hz}, 2 \mathrm{H}, 2^{\prime \prime}, 6^{\prime \prime}-\mathrm{H}\right), 7.36(\mathrm{~d}, J=8.1 \mathrm{~Hz}, 1 \mathrm{H}, 5-\mathrm{H}), 7.39$ (ddd, $J=7.8,7.7,1.1 \mathrm{~Hz}, 1 \mathrm{H}, 7-\mathrm{H}), 7.44$ (s, $1 \mathrm{H}, 1-\mathrm{H}), 7.44$ (d, $J$ $\left.=8.9 \mathrm{~Hz}, 2 \mathrm{H}, 2^{\prime}, 6^{\prime}-\mathrm{H}\right), 7.60(\mathrm{ddd}, J=8.1,7.7,1.7 \mathrm{~Hz}, 1 \mathrm{H}, 6-\mathrm{H})$, $8.29(\mathrm{dd}, J=7.8,1.7 \mathrm{~Hz}, 1 \mathrm{H}, 8-\mathrm{H}) \mathrm{ppm} .{ }^{13} \mathrm{C}$ NMR: $\delta=36.8(\mathrm{C}-$
4), $40.9(\mathrm{C}-3), 55.1\left(4^{\prime \prime}-\mathrm{OCH}_{3}\right), 55.3\left(4^{\prime}-\mathrm{OCH}_{3}\right), 113.8\left(\mathrm{C}-3^{\prime}, 5^{\prime}\right)$, 114.2 (C-3"',5' ), 114.6 (C-1), 117.0 (C-9a), 118.0 (C-5), 123.9 (C8a), 125.0 (C-7), 126.2 (C-8), 126.9 (C-2',6'), $128.4\left(\mathrm{C}-2^{\prime \prime}{ }^{\prime}, 6^{\prime \prime}\right)$, $131.6\left(\mathrm{C}-1^{\prime}\right), 132.7$ (C-1' $), 132.9$ (C-6), 135.3 (C-2), 155.9 (C-4b), 158.6 (C-4' $), 159.1$ (C-4'), 162.1 (C-4a), 174.2 (C-9) ppm. EI-MS: $\mathrm{m} / \mathrm{z}(\%)=410[\mathrm{M}]^{+\cdot}(21), 409(24), 408$ (100), 393 (11), 377 (13), 365 (6), 350 (8), 321 (6), 303 (11), 121 (6). HRMS (EI): calcd. for $\mathrm{C}_{27} \mathrm{H}_{22} \mathrm{O}_{4}[\mathrm{M}]^{+\cdot} 410.1518$; found 410.1526 .

2-(3,4-Dimethoxyphenyl)-3-(4-methoxyphenyl)-9H-xanthen-9-one (5i): M.p. $155-156{ }^{\circ} \mathrm{C} .{ }^{1} \mathrm{H}$ NMR: $\delta=3.63$ (s, $3 \mathrm{H}, 4^{\prime}-\mathrm{OCH}_{3}$ ), 3.81 (s, $\left.3 \mathrm{H}, 4^{\prime \prime}-\mathrm{OCH}_{3}\right), 3.89$ (s, $\left.3 \mathrm{H}, 3^{\prime}-\mathrm{OCH}_{3}\right), 6.60(\mathrm{~d}, J=1.7 \mathrm{~Hz}, 1$ $\left.\mathrm{H}, 2^{\prime}-\mathrm{H}\right), 6.83\left(\mathrm{~d}, J=8.6 \mathrm{~Hz}, 2 \mathrm{H}, 3^{\prime \prime}, 5^{\prime \prime}-\mathrm{H}\right), 6.79-6.85(\mathrm{~m}, 2 \mathrm{H}$, $\left.5^{\prime}, 6^{\prime}-\mathrm{H}\right), 7.15\left(\mathrm{~d}, J=8.6 \mathrm{~Hz}, 2 \mathrm{H}, 2^{\prime \prime}, 6^{\prime \prime}-\mathrm{H}\right), 7.41$ (ddd, $J=8.0$, 7.7, $1.1 \mathrm{~Hz}, 1 \mathrm{H}, 7-\mathrm{H}), 7.52(\mathrm{~d}, J=8.0 \mathrm{~Hz}, 1 \mathrm{H}, 5-\mathrm{H}), 7.53(\mathrm{~s}, 1 \mathrm{H}$, 4-H), 7.75 (ddd, $J=8.0,7.7,1.7 \mathrm{~Hz}, 1 \mathrm{H}, 6-\mathrm{H}), 8.36$ (s, $1 \mathrm{H}, 1-\mathrm{H})$, $8.38(\mathrm{dd}, J=8.0,1.7 \mathrm{~Hz}, 1 \mathrm{H}, 8-\mathrm{H}) \mathrm{ppm} .{ }^{13} \mathrm{C} \mathrm{NMR}: \delta=55.3\left(4^{\prime \prime}{ }^{\prime}\right.$ $\left.\mathrm{OCH}_{3}\right), 55.6\left(4^{\prime}-\mathrm{OCH}_{3}\right), 55.8\left(3^{\prime}-\mathrm{OCH}_{3}\right), 110.8\left(\mathrm{C}-5^{\prime}\right), 113.3(\mathrm{C}-$ $\left.2^{\prime}\right), 113.6$ (C-3' $\left.{ }^{\prime \prime} 5^{\prime \prime}\right), 118.0$ (C-5), 119.2 (C-4), 120.4 (C-9a), 121.9 (C-8a), 122.2 (C-6'), 123.9 (C-7), 126.8 (C-8), 127.9 (C-1), 130.7 $\left(\mathrm{C}-2^{\prime \prime}, 6^{\prime \prime}\right), 132.4\left(\mathrm{C}-1^{\prime \prime}\right), 132.6\left(\mathrm{C}-1^{\prime}\right), 134.7$ (C-6), 136.7 (C-2), 147.3 (C-3), 147.9 and 148.2 (C-3', C-4'), 155.1 (C-4a), 156.3 (C4b), $159.1\left(\mathrm{C}-4^{\prime \prime}\right), 177.0$ (C-9) ppm. EI-MS: $m / z(\%)=438[\mathrm{M}]^{+}$ (100), 423 (8), 407 (9). $\mathrm{C}_{28} \mathrm{H}_{22} \mathrm{O}_{5}$ (438.47): calcd. for $\mathrm{C} 76.70, \mathrm{H}$ 5.06; found C 76.47, H 4.93 .

2-(3,4-Dimethoxyphenyl)-3-(4-methoxyphenyl)-3,4-dihydro-9Hxanthen-9-one (6i): Yellowish oil. ${ }^{1} \mathrm{H}$ NMR: $\delta=2.96(\mathrm{dd}, J=17.3$, $\left.1.6 \mathrm{~Hz}, 1 \mathrm{H}, 4_{\text {trans }}-\mathrm{H}\right), 3.62\left(\mathrm{dd}, J=17.3,8.8 \mathrm{~Hz}, 1 \mathrm{H}, 4_{\text {cis }}-\mathrm{H}\right), 3.71$ $\left(\mathrm{s}, 3 \mathrm{H}, 4^{\prime \prime}{ }^{\prime}-\mathrm{OCH}_{3}\right), 3.84$ and $3.86\left(2 \times \mathrm{s}, 6 \mathrm{H}, 3^{\prime}, 4^{\prime}-\mathrm{OCH}_{3}\right), 4.24$ (brd, $J=8.8 \mathrm{~Hz}, 1 \mathrm{H}, 3-\mathrm{H}), 6.75$ (d, $\left.J=8.5 \mathrm{~Hz}, 1 \mathrm{H}, 5^{\prime}-\mathrm{H}\right), 6.77$ (d, $\left.J=8.7 \mathrm{~Hz}, 2 \mathrm{H}, 3^{\prime \prime}, 5^{\prime \prime}-\mathrm{H}\right), 6.97$ (dd, $J=8.5,2.2 \mathrm{~Hz}, 1 \mathrm{H}, 6^{\prime}-$ $\mathrm{H}), 7.10\left(\mathrm{~d}, J=2.2 \mathrm{~Hz}, 1 \mathrm{H}, 2^{\prime}-\mathrm{H}\right), 7.21\left(\mathrm{~d}, J=8.7 \mathrm{~Hz}, 2 \mathrm{H}, 2^{\prime \prime}, 6^{\prime \prime}\right.$ $\mathrm{H}), 7.35(\mathrm{dd}, J=8.3,1.0 \mathrm{~Hz}, 1 \mathrm{H}, 5-\mathrm{H}), 7.38(\mathrm{ddd}, J=7.8,7.7$, $1.0 \mathrm{~Hz}, 1 \mathrm{H}, 7-\mathrm{H}), 7.44$ (s, $1 \mathrm{H}, 1-\mathrm{H}), 7.59$ (ddd, $J=8.3,7.7,1.7 \mathrm{~Hz}$, $1 \mathrm{H}, 6-\mathrm{H}), 8.29(\mathrm{dd}, J=7.8,1.7 \mathrm{~Hz}, 1 \mathrm{H}, 8-\mathrm{H}) \mathrm{ppm} .{ }^{13} \mathrm{C} \mathrm{NMR}: \delta$ $=36.7(\mathrm{C}-4), 41.1(\mathrm{C}-3), 55.1\left(4^{\prime \prime}-\mathrm{OCH}_{3}\right), 55.81$ and $55.82\left(3^{\prime}, 4^{\prime}-\right.$ $\left.\mathrm{OCH}_{3}\right), 108.7\left(\mathrm{C}-2^{\prime}\right), 110.8\left(\mathrm{C}-5^{\prime}\right), 114.2\left(\mathrm{C}-3^{\prime \prime}{ }^{\prime}, 5^{\prime \prime}\right), 114.8(\mathrm{C}-1)$, 116.9 (C-9a), 118.0 (C-5), 118.2 (C-6'), 123.8 (C-8a), 125.0 (C-7), 126.1 (C-8), $128.3\left(\mathrm{C}-2^{\prime \prime}, 6^{\prime \prime}\right), 132.0\left(\mathrm{C}-1^{\prime}\right), 132.8$ (C-1' $), 132.9$ (C-

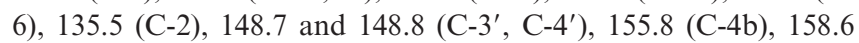
$\left(\mathrm{C}-4^{\prime \prime}\right), 162.2(\mathrm{C}-4 \mathrm{a}), 174.2(\mathrm{C}-9) \mathrm{ppm}$. EI-MS: $m / z(\%)=440$ $[\mathrm{M}]^{+\cdot}(100), 439$ (29), 438 (53), 423 (11), 391 (8), 333 (10), 303 (13), 165 (47), 151 (14), 121 (8), 86 (8), 84 (13). HRMS (EI): calcd. for $\mathrm{C}_{28} \mathrm{H}_{24} \mathrm{O}_{5}[\mathrm{M}]^{+\cdot} 440.1624$; found 440.1623 .

3-(3,4-Dimethoxyphenyl)-2-phenyl-9H-xanthen-9-one (5j): M.p. 212-214 ${ }^{\circ} \mathrm{C} .{ }^{1} \mathrm{H}$ NMR: $\delta=3.52\left(\mathrm{~s}, 3 \mathrm{H}, 3^{\prime \prime}-\mathrm{OCH}_{3}\right), 3.89(\mathrm{~s}, 3 \mathrm{H}$, $\left.4^{\prime \prime}-\mathrm{OCH}_{3}\right), 6.56\left(\mathrm{~d}, J=2.0 \mathrm{~Hz}, 1 \mathrm{H}, 2^{\prime \prime}-\mathrm{H}\right), 6.83(\mathrm{~d}, J=8.3 \mathrm{~Hz}, 1$ $\left.\mathrm{H}, 5^{\prime \prime}-\mathrm{H}\right), 6.92\left(\mathrm{dd}, J=8.3,2.0 \mathrm{~Hz}, 1 \mathrm{H}, 6^{\prime \prime}-\mathrm{H}\right), 7.18-7.21(\mathrm{~m}, 2$ H, 2', $\left.6^{\prime}-\mathrm{H}\right), 7.24-7.28\left(\mathrm{~m}, 3 \mathrm{H}, 3^{\prime}, 4^{\prime}, 5^{\prime}-\mathrm{H}\right), 7.40$ (ddd, $J=7.8,7.6$, $1.0 \mathrm{~Hz}, 1 \mathrm{H}, 7-\mathrm{H}), 7.52(\mathrm{~d}, J=8.0 \mathrm{~Hz}, 1 \mathrm{H}, 5-\mathrm{H}), 7.58$ (s, $1 \mathrm{H}, 4-$ H), 7.74 (ddd, $J=8.0,7.6,1.7 \mathrm{~Hz}, 1 \mathrm{H}, 6-\mathrm{H}), 8.35(\mathrm{~s}, 1 \mathrm{H}, 1-\mathrm{H})$, $8.37(\mathrm{dd}, J=7.8,1.7 \mathrm{~Hz}, 1 \mathrm{H}, 8-\mathrm{H}) \mathrm{ppm} .{ }^{13} \mathrm{C} \mathrm{NMR}: \delta=55.5\left(3^{\prime \prime}{ }_{-}\right.$ $\left.\mathrm{OCH}_{3}\right), 55.8\left(4^{\prime \prime}-\mathrm{OCH}_{3}\right), 110.7\left(\mathrm{C}-5^{\prime \prime}\right), 113.3\left(\mathrm{C}-2^{\prime \prime}\right), 117.9(\mathrm{C}-5)$, 119.0 (C-4), 120.4 (C-9a), 121.89 (C-6"'), 121.93 (C-8a), 123.9 (C7), 126.7 (C-8), 126.8 (C-4'), 128.1 (C-3',5'), 128.3 (C-1), 129.8 (C$\left.2^{\prime}, 6^{\prime}\right), 132.2\left(\mathrm{C}-1^{\prime \prime}\right), 134.7$ (C-6), 137.0 (C-2), 140.2 (C-1'), 147.2 (C-3), 148.1 and 148.6 (C-3"', C-4"'), 155.3 (C-4a), 156.2 (C-4b), 176.9 (C-9) ppm. EI-MS: $m / z(\%)=408\left[^{\mathrm{M}}\right]^{+\cdot}(100), 393(15), 377$ 814, 361 (13), 350 (10), 333 (15), 321 (10), 305 (8), 292 (6). $\mathrm{C}_{27} \mathrm{H}_{20} \mathrm{O}_{4}$ (408.45): calcd. for $\mathrm{C} 79.40, \mathrm{H} \mathrm{4.94}$; found $\mathrm{C} 79.07, \mathrm{H}$ 4.91 .

3-(3,4-Dimethoxyphenyl)-2-phenyl-3,4-dihydro-9H-xanthen-9-one (6j): Yellowish oil. ${ }^{1} \mathrm{H}$ NMR: $\delta=2.99(\mathrm{dd}, J=17.3,1.5 \mathrm{~Hz}, 1 \mathrm{H}$, 
$\left.4_{\text {trans }}-\mathrm{H}\right), 3.63\left(\mathrm{dd}, J=17.3,8.9 \mathrm{~Hz}, 1 \mathrm{H}, 4_{\text {cis }}-\mathrm{H}\right), 3.77\left(\mathrm{~s}, 3 \mathrm{H}, 3^{\prime \prime}\right.$ $\left.\mathrm{OCH}_{3}\right), 3.79\left(\mathrm{~s}, 3 \mathrm{H}, 4^{\prime \prime}-\mathrm{OCH}_{3}\right), 4.25(\mathrm{dd}, J=8.9,1.5 \mathrm{~Hz}, 1 \mathrm{H}, 3-$ $\mathrm{H}), 6.71\left(\mathrm{~d}, J=8.2 \mathrm{~Hz}, 1 \mathrm{H}, 5^{\prime \prime}-\mathrm{H}\right), 6.82\left(\mathrm{~d}, J=2.0 \mathrm{~Hz}, 1 \mathrm{H}, 2^{\prime \prime}-\right.$ $\mathrm{H}), 6.85\left(\mathrm{dd}, J=8.2,2.0 \mathrm{~Hz}, 1 \mathrm{H}, 6^{\prime \prime}-\mathrm{H}\right), 7.22-7.32(\mathrm{~m}, 3 \mathrm{H}$, $\left.3^{\prime}, 4^{\prime}, 5^{\prime}-\mathrm{H}\right), 7.36(\mathrm{~d}, J=8.0 \mathrm{~Hz}, 1 \mathrm{H}, 5-\mathrm{H}), 7.38(\mathrm{ddd}, J=7.8,7.7$, $1.3 \mathrm{~Hz}, 1 \mathrm{H}, 7-\mathrm{H}), 7.47-7.50$ (m, $\left.2 \mathrm{H}, 2^{\prime}, 6^{\prime}-\mathrm{H}\right), 7.54$ (s, $\left.1 \mathrm{H}, 1-\mathrm{H}\right)$, 7.60 (ddd, $J=8.0,7.7,1.6 \mathrm{~Hz}, 1 \mathrm{H}, 6-\mathrm{H}), 8.28$ (dd, $J=7.8,1.6 \mathrm{~Hz}$, $1 \mathrm{H}, 8-\mathrm{H})$ ppm. ${ }^{13} \mathrm{C}$ NMR: $\delta=37.0(\mathrm{C}-4), 41.3(\mathrm{C}-3), 55.7$ and $55.8\left(3^{\prime \prime}, 4^{\prime \prime}-\mathrm{OCH}_{3}\right), 110.3\left(\mathrm{C}-2^{\prime \prime}\right), 111.4\left(\mathrm{C}-5^{\prime \prime}\right), 116.6(\mathrm{C}-1), 116.7$ (C-9a), 118.0 (C-5), 119.2 (C-6"'), 123.8 (C-8a), 125.0 (C-7), 125.6 (C-2',6'), 126.1 (C-8), $127.5\left(\mathrm{C}-4^{\prime}\right), 128.4\left(\mathrm{C}-3^{\prime}, 5^{\prime}\right), 132.99$ and

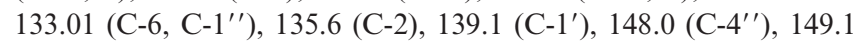
$\left(\mathrm{C}-3^{\prime \prime}\right), 155.8$ (C-4b), 162.6 (C-4a), 174.1 (C-9) ppm. EI-MS: m/z $(\%)=410[\mathrm{M}]^{+\cdot}(100), 409$ (53), 408 (73), 395 (18), 393 (19), 379 (10), 377 (12), 361 (18), 334 (16), 333 (46), 321 (11), 299 (31), 273 (27), 215 (10), 202 (8), 165 (12), 151 (11), 105 (14), 86 (17), 84 (26). HRMS (EI): calcd. for $\mathrm{C}_{27} \mathrm{H}_{22} \mathrm{O}_{4}[\mathrm{M}]^{+*}$ 410.1518; found 410.1515 .

3-(3,4-Dimethoxyphenyl)-2-(4-methoxyphenyl)-9H-xanthen-9-one (5k) and 3-(3,4-Dimethoxyphenyl)-2-(4-methoxyphenyl)-3,4-dihydro$\mathbf{9 H}$-xanthen-9-one (6k): These compounds showed spectroscopic and analytical data identical to those previously reported. ${ }^{[27]}$

2,3-Bis(3,4-dimethoxyphenyl)-9H-xanthen-9-one (5l): M.p. 171$173{ }^{\circ} \mathrm{C} .{ }^{1} \mathrm{H}$ NMR: $\delta=3.63$ and $3.66\left(2 \times \mathrm{s}, 6 \mathrm{H}, 4^{\prime}, 4^{\prime \prime}-\mathrm{OCH}_{3}\right), 3.88$ and $3.89\left(2 \times \mathrm{s}, 6 \mathrm{H}, 3^{\prime}, 3^{\prime \prime}-\mathrm{OCH}_{3}\right), 6.63$ (brs, $\left.2 \mathrm{H}, 2^{\prime}-\mathrm{H}, 2^{\prime \prime}-\mathrm{H}\right)$, 6.80-6.89 (m, $\left.4 \mathrm{H}, 5^{\prime}-\mathrm{H}, 6^{\prime}-\mathrm{H}, 5^{\prime \prime}-\mathrm{H}, 6^{\prime \prime}-\mathrm{H}\right), 7.41(\mathrm{dd}, J=8.2$, $7.7 \mathrm{~Hz}, 1 \mathrm{H}, 7-\mathrm{H}), 7.52$ (d, $J=8.1 \mathrm{~Hz}, 1 \mathrm{H}, 5-\mathrm{H}), 7.56$ (s, $1 \mathrm{H}, 4-$ H), 7.75 (ddd, $J=8.1,7.7,1.6 \mathrm{~Hz}, 1 \mathrm{H}, 6-\mathrm{H}), 8.36$ (s, $1 \mathrm{H}, 1-\mathrm{H})$, $8.38(\mathrm{dd}, J=8.2,1.6 \mathrm{~Hz}, 1 \mathrm{H}, 8-\mathrm{H}) \mathrm{ppm} .{ }^{13} \mathrm{C}$ NMR: $\delta=55.69$ and $55.71\left(4^{\prime}, 4^{\prime \prime}-\mathrm{OCH}_{3}\right), 55.8\left(3^{\prime}, 3^{\prime \prime}-\mathrm{OCH}_{3}\right), 110.8\left(\mathrm{C}-5^{\prime}, \mathrm{C}-5^{\prime \prime}\right), 113.0$ $\left(\mathrm{C}-2^{\prime \prime}\right), 113.2$ (C-2'), 118.0 (C-5), 119.1 (C-4), 120.4 (C-9a), 121.9 (C-6', C-6"'), 122.2 (C-8a), 123.9 (C-7), 126.7 (C-8), 128.0 (C-1), 132.5 and $132.7\left(\mathrm{C}-1^{\prime}, \mathrm{C}-1^{\prime \prime}\right), 134.8$ (C-6), 136.7 (C-2), 147.3 (C3), 148.0, 148.3 and 148.6 (C-3', C-4', C-3' 'and C-4' '), 155.1 (C4a), 156.3 (C-4b), 177.0 (C-9) ppm. EI-MS: $m / z(\%)=468[\mathrm{M}]^{+}$ (100), 453 (10), 379 (5), 97 (6). $\mathrm{C}_{29} \mathrm{H}_{24} \mathrm{O}_{6}$ (468.50): calcd. for $\mathrm{C}$ 74.35, H 5.16; found C 74.25, H 5.22.

2,3-Bis(3,4-dimethoxyphenyl)-3,4-dihydro-9H-xanthen-9-one (6I): Yellowish oil. ${ }^{1} \mathrm{H}$ NMR: $\delta=2.98\left(\mathrm{dd}, J=17.3,1.5 \mathrm{~Hz}, 1 \mathrm{H}, 4\right.$ trans $^{-}$ $\mathrm{H}), 3.63\left(\mathrm{dd}, J=17.3,8.3 \mathrm{~Hz}, 1 \mathrm{H}, 4_{\text {cis }}-\mathrm{H}\right), 3.77$ and $3.79(2 \times \mathrm{s}, 6$ $\left.\mathrm{H}, 4^{\prime}, 4^{\prime \prime}-\mathrm{OCH}_{3}\right), 3.85$ and $3.86\left(2 \times \mathrm{s}, 6 \mathrm{H}, 3^{\prime}, 3^{\prime \prime}-\mathrm{OCH}_{3}\right), 4.23$ (brd, $J=8.3 \mathrm{~Hz}, 1 \mathrm{H}, 3-\mathrm{H}), 6.72\left(\mathrm{~d}, J=8.0 \mathrm{~Hz}, 1 \mathrm{H}, 5^{\prime \prime}-\mathrm{H}\right), 6.76(\mathrm{~d}, J$ $\left.=8.4 \mathrm{~Hz}, 1 \mathrm{H}, 5^{\prime}-\mathrm{H}\right), 6.83\left(\mathrm{~d}, J=2.0 \mathrm{~Hz}, 1 \mathrm{H}, 2^{\prime \prime}-\mathrm{H}\right), 6.85(\mathrm{dd}, J$ $\left.=8.0,2.0 \mathrm{~Hz}, 1 \mathrm{H}, 6^{\prime \prime}-\mathrm{H}\right), 6.98\left(\mathrm{dd}, J=8.4,2.1 \mathrm{~Hz}, 1 \mathrm{H}, 6^{\prime}-\mathrm{H}\right)$, $7.11\left(\mathrm{~d}, J=2.1 \mathrm{~Hz}, 1 \mathrm{H}, 2^{\prime}-\mathrm{H}\right), 7.36(\mathrm{dd}, J=8.5,1.0 \mathrm{~Hz}, 1 \mathrm{H}, 5-$ $\mathrm{H}), 7.38(\mathrm{dt}, J=7.7,1.0 \mathrm{~Hz}, 1 \mathrm{H}, 7-\mathrm{H}), 7.45(\mathrm{~s}, 1 \mathrm{H}, 1-\mathrm{H}), 7.60$ (ddd, $J=8.5,7.7,1.7 \mathrm{~Hz}, 1 \mathrm{H}, 6-\mathrm{H}), 8.28(\mathrm{dd}, J=7.7,1.7 \mathrm{~Hz}, 1$ $\mathrm{H}, 8-\mathrm{H})$ ppm. ${ }^{13} \mathrm{C}$ NMR: $\delta=36.7$ (C-4), 41.5 (C-3), 55.7, 55.77 and $55.80\left(3^{\prime}, 4^{\prime}, 3^{\prime \prime}, 4^{\prime \prime}-\mathrm{OCH}_{3}\right), 108.7\left(\mathrm{C}-2^{\prime}\right), 110.2\left(\mathrm{C}-2^{\prime \prime}\right), 110.8$ (C-5'), 111.4 (C-5' ), 114.9 (C-1), 116.8 (C-9a), 117.9 (C-5), 118.2 (C-6'), 119.1 (C-6"'), 123.7 (C-8a), 125.0 (C-7), 126.1 (C-8), 132.0 (C-1'), 133.0 (C-6), 133.2 (C-1' '), 135.4 (C-2), 148.0, 148.7 and 149.1 (C-3', C-4', C-3' 'and C-4' '), 155.8 (C-4b), 162.3 (C-4a), 174.1 (C-9) ppm. EI-MS: $m / z(\%)=470[\mathrm{M}]^{+*}(78), 469(28), 468$ (100), 455 (7), 453 (10), 421 (7), 333 (16), 332 (7), 84 (7). HRMS (EI): calcd. for $\mathrm{C}_{29} \mathrm{H}_{26} \mathrm{O}_{6}[\mathrm{M}]^{+\cdot} 470.1729$; found 470.1739 .

6-Methoxy-2,3-diphenyl-9H-xanthen-9-one (5m): M.p. $151-153{ }^{\circ} \mathrm{C}$. ${ }^{1} \mathrm{H}$ NMR: $\delta=3.95$ (s, $\left.3 \mathrm{H}, 6-\mathrm{OCH}_{3}\right), 6.91$ (d, $J=2.4 \mathrm{~Hz}, 1 \mathrm{H}, 5-$ $\mathrm{H}), 6.96(\mathrm{dd}, J=8.9,2.4 \mathrm{~Hz}, 1 \mathrm{H}, 7-\mathrm{H}), 7.15-7.28(\mathrm{~m}, 10 \mathrm{H}, 2,3-$ $\left.\mathrm{C}_{6} H_{5}\right), 7.52(\mathrm{~s}, 1 \mathrm{H}, 4-\mathrm{H}), 8.28(\mathrm{~d}, J=8.9 \mathrm{~Hz}, 1 \mathrm{H}, 8-\mathrm{H}), 8.36(\mathrm{~s}$, $1 \mathrm{H}, 1-\mathrm{H}) \mathrm{ppm} .{ }^{13} \mathrm{C}$ NMR: $\delta=55.8\left(6-\mathrm{OCH}_{3}\right), 100.2(\mathrm{C}-5), 113.3$ (C-7), 115.9 (C-8a), 119.3 (C-4), 120.8 (C-9a), 126.8 (C-4'), 127.5 $\left(\mathrm{C}-4^{\prime \prime}\right), 128.0\left(\mathrm{C}-3^{\prime}, 5^{\prime}\right), 128.1\left(\mathrm{C}-3^{\prime \prime}, 5^{\prime \prime}\right), 128.3(\mathrm{C}-1, \mathrm{C}-8), 129.6$
$\left(\mathrm{C}-2^{\prime \prime}, 6^{\prime \prime}\right), 129.9\left(\mathrm{C}-2^{\prime}, 6^{\prime}\right), 137.0(\mathrm{C}-2), 139.88$ and $139.94\left(\mathrm{C}-1^{\prime}\right.$, C-1"'), 147.0 (C-3), 155.2 (C-4a), 158.2 (C-4b), 165.0 (C-6), 176.0 (C-9) ppm. EI-MS: $m / z(\%)=378[\mathrm{M}]^{+*}(100), 377$ (63), 363 (13), 335 (7), 334 (16), 277 (5), 276 (7), 228 (5), 226 (5). $\mathrm{C}_{26} \mathrm{H}_{18} \mathrm{O}_{3}$ (378.42): calcd. for C 82.52, H 4.79; found C 82.56, H 4.92 .

6-Methoxy-2,3-diphenyl-3,4-dihydro-9H-xanthen-9-one (6m): Yellowish oil. ${ }^{1} \mathrm{H}$ NMR: $\delta=2.96\left(\mathrm{dd}, J=17.3,1.5 \mathrm{~Hz}, 1 \mathrm{H}, 4_{\text {trans }}-\mathrm{H}\right)$, $3.64\left(\mathrm{dd}, J=17.3,9.0 \mathrm{~Hz}, 1 \mathrm{H}, 4_{c i s}-\mathrm{H}\right), 3.85\left(\mathrm{~s}, 3 \mathrm{H}, 6-\mathrm{OCH}_{3}\right), 4.28$ $(\mathrm{dd}, J=9.0,1.5 \mathrm{~Hz}, 1 \mathrm{H}, 3-\mathrm{H}), 6.74(\mathrm{~d}, J=2.4 \mathrm{~Hz}, 1 \mathrm{H}, 5-\mathrm{H}), 6.95$ (dd, $J=8.9,2.4 \mathrm{~Hz}, 1 \mathrm{H}, 7-\mathrm{H}), 7.18-7.32$ (m, $8 \mathrm{H}, 3^{\prime}, 4^{\prime}, 5^{\prime}-\mathrm{H}, 3-$ $\left.\mathrm{C}_{6} H_{5}\right), 7.45-7.48\left(\mathrm{~m}, 2 \mathrm{H}, 2^{\prime}, 6^{\prime}-\mathrm{H}\right), 7.54(\mathrm{~s}, 1 \mathrm{H}, 1-\mathrm{H}), 8.18$ (d, $J=$ $8.9 \mathrm{~Hz}, 1 \mathrm{H}, 8-\mathrm{H}) \mathrm{ppm} .{ }^{13} \mathrm{C}$ NMR: $\delta=36.6$ (C-4), 41.7 (C-3), 55.7 $\left(6-\mathrm{OCH}_{3}\right), 100.4(\mathrm{C}-5), 114.2(\mathrm{C}-7), 116.6(\mathrm{C}-9 \mathrm{a}), 116.9$ (C-1), 117.7 (C-8a), $125.6\left(\mathrm{C}-2^{\prime}, 6^{\prime}\right), 127.1\left(\mathrm{C}-4^{\prime}\right), 127.3\left(\mathrm{C}-2^{\prime \prime}, 6^{\prime \prime}\right), 127.46$ and 127.49 (C-8, C-4' $), 128.4$ (C-3',5'), 128.9 (C-3'",5' $), 135.0$ (C-2),

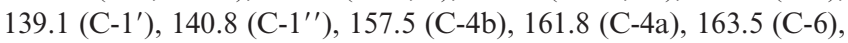
173.8 (C-9) ppm. EI-MS: $m / z(\%)=380[\mathrm{M}]^{+\cdot}(8), 379(20), 378$ (100), 377 (51), 363 (11), 334 (11), 303 (8). HRMS (EI): calcd. for $\mathrm{C}_{26} \mathrm{H}_{20} \mathrm{O}_{3}[\mathrm{M}]^{+*}$ 380.1412; found 380.1444.

1-Methoxy-6,7-diphenyl-9H-xanthen-9-one (5n): M.p. $203-205^{\circ} \mathrm{C}$. ${ }^{1} \mathrm{H}$ NMR: $\delta=4.04\left(\mathrm{~s}, 3 \mathrm{H}, 8-\mathrm{OCH}_{3}\right), 6.82(\mathrm{dd}, J=8.4,0.7 \mathrm{~Hz}, 1$ $\mathrm{H}, 7-\mathrm{H}), 7.07(\mathrm{dd}, J=8.4,0.7 \mathrm{~Hz}, 1 \mathrm{H}, 5-\mathrm{H}), 7.14-7.27(\mathrm{~m}, 10 \mathrm{H}$, 2,3- $\left.\mathrm{C}_{6} H_{5}\right), 7.47$ (s, $\left.1 \mathrm{H}, 4-\mathrm{H}\right), 7.61(\mathrm{t}, J=8.4 \mathrm{~Hz}, 1 \mathrm{H}, 6-\mathrm{H}), 8.33$ (s, $1 \mathrm{H}, 1-\mathrm{H}) \mathrm{ppm} .{ }^{13} \mathrm{C}$ NMR: $\delta=56.4\left(8-\mathrm{OCH}_{3}\right), 105.4(\mathrm{C}-7)$, 110.0 (C-5), 112.6 (C-8a), 118.9 (C-4), 121.8 (C-9a), 126.7 (C-4'), $127.4\left(\mathrm{C}-4^{\prime \prime}\right), 127.9\left(\mathrm{C}-3^{\prime}, 5^{\prime}\right), 128.0\left(\mathrm{C}-3^{\prime \prime}, 5^{\prime \prime}\right), 128.4(\mathrm{C}-1), 129.6$ $\left(\mathrm{C}-2^{\prime \prime}, 6^{\prime \prime}\right), 129.9\left(\mathrm{C}-2^{\prime}, 6^{\prime}\right), 134.8$ (C-6), 136.8 (C-2), 139.9 (C-1', C$\left.1^{\prime \prime}\right), 146.9$ (C-3), 154.1 (C-4a), 158.2 (C-4b), 160.7 (C-8), 176.3 (C9) ppm. EI-MS: $m / z(\%)=378[\mathrm{M}]^{+\cdot}(100), 377(23), 361(10), 360$ (8), 350 (9), 349 (60), 347 (10), 333 (13), 332 (42), 301 (6), 289 (8), 276 (6). $\mathrm{C}_{26} \mathrm{H}_{18} \mathrm{O}_{3}$ (378.42): calcd. for $\mathrm{C} 82.52, \mathrm{H} \mathrm{4.79}$; found $\mathrm{C}$ 82.27, H 4.73 .

1-Methoxy-6,7-diphenyl-7,8-dihydro-9H-xanthen-9-one (6n): M.p. 169-172 ${ }^{\circ} \mathrm{C} .{ }^{1} \mathrm{H}$ NMR: $\delta=2.91\left(\mathrm{dd}, J=17.3,1.5 \mathrm{~Hz}, 1 \mathrm{H}, 4_{\text {trans }}{ }^{-}\right.$ H), $3.59\left(\mathrm{dd}, J=17.3,9.1 \mathrm{~Hz}, 1 \mathrm{H}, 4_{c i s}-\mathrm{H}\right), 3.98\left(\mathrm{~s}, 3 \mathrm{H}, 8-\mathrm{OCH}_{3}\right)$, $4.27(\mathrm{dd}, J=9.1,1.5 \mathrm{~Hz}, 1 \mathrm{H}, 3-\mathrm{H}), 6.77(\mathrm{~d}, J=8.3 \mathrm{~Hz}, 1 \mathrm{H}, 7-$ $\mathrm{H}), 6.89$ (d, $J=8.3 \mathrm{~Hz}, 1 \mathrm{H}, 5-\mathrm{H}), 7.17-7.31\left(\mathrm{~m}, 8 \mathrm{H}, 3^{\prime}, 4^{\prime}, 5^{\prime}-\mathrm{H}\right.$, $\left.3-\mathrm{C}_{6} H_{5}\right), 7.45(\mathrm{t}, J=8.3 \mathrm{~Hz}, 1 \mathrm{H}, 6-\mathrm{H}), 7.44-7.47$ (m, $2 \mathrm{H}, 2^{\prime}, 6^{\prime}-$ $\mathrm{H}), 7.55(\mathrm{~s}, 1 \mathrm{H}, 1-\mathrm{H}) \mathrm{ppm} .{ }^{13} \mathrm{C}$ NMR: $\delta=36.2(\mathrm{C}-4), 41.7(\mathrm{C}-3)$, $56.3\left(8-\mathrm{OCH}_{3}\right), 106.3$ (C-7), 110.1 (C-5), 114.4 (C-8a), 117.0 (C-1), 117.8 (C-9a), $125.8\left(\mathrm{C}-2^{\prime}, 6^{\prime}\right), 127.1$ and $127.4\left(\mathrm{C}-4^{\prime}, \mathrm{C}-4^{\prime \prime}\right), 1273$. $\left(\mathrm{C}-2^{\prime \prime}, 6^{\prime \prime}\right), 128.4\left(\mathrm{C}-3^{\prime}, 5^{\prime}\right), 128.9\left(\mathrm{C}-3^{\prime \prime}, 5^{\prime \prime}\right), 133.0$ (C-6), 134.8 (C2), $139.2\left(\mathrm{C}-1^{\prime}\right), 140.8\left(\mathrm{C}-1^{\prime \prime}\right), 157.8(\mathrm{C}-4 \mathrm{~b}), 160.09$ and $161.13(\mathrm{C}-$ 8, C-4a), 173.9 (C-9) ppm. EI-MS: $m / z(\%)=380[\mathrm{M}]^{+*}(58), 379$ (28), 378 (100), 377 (28), 363 (13), 362 (11), 361 (23), 360 (11), 350 (14), 349 (69), 347 (15), 333 (20), 332 (46), 303 (17), 301 (11), 289 (21), 288 (14), 285 (14), 266 (27), 261 (10), 226 (9), 215 (8). HRMS (EI): calcd. for $\mathrm{C}_{26} \mathrm{H}_{20} \mathrm{O}_{3}[\mathrm{M}]^{+\cdot} 380.1412$; found 380.1418 .

1-Hydroxy-6,7-diphenyl-9H-xanthen-9-one (12n): M.p. $160-162{ }^{\circ} \mathrm{C}$. ${ }^{1} \mathrm{H}$ NMR: $\delta=6.79(\mathrm{dd}, J=8.4,0.6 \mathrm{~Hz}, 1 \mathrm{H}, 7-\mathrm{H}), 6.91(\mathrm{dd}, J=$ 8.4, 0.6 Hz, $1 \mathrm{H}, 5-\mathrm{H}), 7.12-7.16\left(\mathrm{~m}, 2 \mathrm{H}, 2^{\prime}, 6^{\prime}-\mathrm{H}\right), 7.17-7.20$ (m, $\left.2 \mathrm{H}, 2^{\prime \prime}, 6^{\prime \prime}-\mathrm{H}\right), 7.22-7.28$ (m, $\left.6 \mathrm{H}, 3^{\prime}, 4^{\prime}, 5^{\prime}-\mathrm{H}, 3^{\prime \prime}, 4^{\prime \prime}, 5^{\prime \prime}-\mathrm{H}\right), 7.49$ (s, $1 \mathrm{H}, 4-\mathrm{H}), 7.57$ (t, $J=8.4 \mathrm{~Hz}, 1 \mathrm{H}, 6-\mathrm{H}), 8.26(\mathrm{~s}, 1 \mathrm{H}, 1-\mathrm{H})$, $12.67(\mathrm{~s}, 1 \mathrm{H}, 8-\mathrm{OH})$ ppm. ${ }^{13} \mathrm{C}$ NMR: $\delta=107.0(\mathrm{C}-5), 109.0(\mathrm{C}-$ 8a), 110.5 (C-7), 119.3 (C-4, C-9a), 127.0 and 127.5 (C-4', C-4' $)$, $127.7(\mathrm{C}-1), 128.0$ and $128.1\left(\mathrm{C}-3^{\prime}, 5^{\prime}, \mathrm{C}-3^{\prime \prime}, 5^{\prime \prime}\right), 129.5\left(\mathrm{C}-2^{\prime \prime}, 6^{\prime \prime}\right)$, $129.8\left(\mathrm{C}-2^{\prime}, 6^{\prime}\right), 136.7$ (C-6), $137.2(\mathrm{C}-2), 139.5$ and 139.6 (C-1', C$\left.1^{\prime \prime}\right), 148.4$ (C-3), 155.1 (C-4a), 156.3 (C-4b), 161.9 (C-8), 182.0 (C9) ppm. EI-MS: $m / z(\%)=378[\mathrm{M}]^{+\cdot}(100), 365(16), 363(40), 349$ (12), 289 (6), 228 (6). HRMS (EI): calcd. for $\mathrm{C}_{25} \mathrm{H}_{16} \mathrm{O}_{3}[\mathrm{M}]^{+}$. 364.1099 ; found 364.1102 . 
1-Methoxy-7-(4-methoxyphenyl)-6-phenyl-9H-xanthen-9-one (50): M.p. $192-194{ }^{\circ} \mathrm{C} .{ }^{1} \mathrm{H}$ NMR: $\delta=3.79\left(\mathrm{~s}, 3 \mathrm{H}, 4^{\prime}-\mathrm{OCH}_{3}\right), 4.04$ (s, 3 $\left.\mathrm{H}, 8-\mathrm{OCH}_{3}\right), 6.78\left(\mathrm{~d}, J=8.8 \mathrm{~Hz}, 2 \mathrm{H}, 3^{\prime}, 5^{\prime}-\mathrm{H}\right), 6.82(\mathrm{~d}, J=8.3 \mathrm{~Hz}$, $1 \mathrm{H}, 7-\mathrm{H}), 7.08$ (d, $\left.J=8.8 \mathrm{~Hz}, 2 \mathrm{H}, 2^{\prime}, 6^{\prime}-\mathrm{H}\right), 7.08$ (dd, $J=8.3$, $0.8 \mathrm{~Hz}, 1 \mathrm{H}, 5-\mathrm{H}), 7.18-7.21\left(\mathrm{~m}, 2 \mathrm{H}, 2^{\prime \prime}, 6^{\prime \prime}-\mathrm{H}\right), 7.27-7.29$ (m, 3 H, 3" , 4" , 5" $-\mathrm{H}), 7.46$ (s, $1 \mathrm{H}, 4-\mathrm{H}), 7.62$ (t, $J=8.3 \mathrm{~Hz}, 1 \mathrm{H}, 6-\mathrm{H})$, $8.30(\mathrm{~s}, 1 \mathrm{H}, 1-\mathrm{H}) \mathrm{ppm} .{ }^{13} \mathrm{C} \mathrm{NMR}: \delta=55.2\left(4^{\prime}-\mathrm{OCH}_{3}\right), 56.5(8-$ $\left.\mathrm{OCH}_{3}\right), 105.4(\mathrm{C}-7), 110.1(\mathrm{C}-5), 112.6(\mathrm{C}-8 \mathrm{a}), 113.4\left(\mathrm{C}-3^{\prime}, 5^{\prime}\right)$, 118.9 (C-4), 121.9 (C-9a), 127.4 (C-4" ), 128.1 (C-3" ,5' $), 128.2$ (C1), $129.6\left(\mathrm{C}-2^{\prime \prime}, 6^{\prime \prime}\right), 131.0\left(\mathrm{C}-2^{\prime}, 6^{\prime}\right), 132.4\left(\mathrm{C}-1^{\prime}\right), 134.8$ (C-6), 136.5

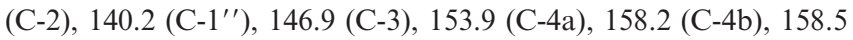
$\left(\mathrm{C}-4^{\prime}\right), 160.8(\mathrm{C}-8), 176.4$ (C-9) ppm. EI-MS: $m / z(\%)=408[\mathrm{M}]^{+*}$ (100), 407 (23), 391 (14), 390 (9), 380 (10), 379 (41), 363 (14), 362 (32), 347 (10), 346 (6), 305 (5). $\mathrm{C}_{27} \mathrm{H}_{20} \mathrm{O}_{4}$ (408.45): calcd. for $\mathrm{C}$ 79.40, H 4.94; found C 79.42, H 4.91.

1-Methoxy-7-(4-methoxyphenyl)-6-phenyl-7,8-dihydro-9H-xanthen9-one (6o): M.p. $168-170{ }^{\circ} \mathrm{C} .{ }^{1} \mathrm{H}$ NMR: $\delta=2.89$ (dd, $J=17.3$, $\left.1.5 \mathrm{~Hz}, 1 \mathrm{H}, 4_{\text {trans }}-\mathrm{H}\right), 3.58\left(\mathrm{dd}, J=17.3 .8 .5 \mathrm{~Hz}, 1 \mathrm{H}, 4_{\text {cis }}-\mathrm{H}\right), 3.76$ (s, $3 \mathrm{H}, 4^{\prime}-\mathrm{OCH}_{3}$ ), 3.98 (s, $3 \mathrm{H}, 8-\mathrm{OCH}_{3}$ ), 4.24 (brd, $J=8.5 \mathrm{~Hz}, 1$ $\mathrm{H}, 3-\mathrm{H}), 6.76(\mathrm{~d}, J=8.4 \mathrm{~Hz}, 1 \mathrm{H}, 7-\mathrm{H}), 6.80(\mathrm{~d}, J=8.9 \mathrm{~Hz}, 2 \mathrm{H}$, $\left.3^{\prime}, 5^{\prime}-\mathrm{H}\right), 6.89(\mathrm{dd}, J=8.4,0.7 \mathrm{~Hz}, 1 \mathrm{H}, 5-\mathrm{H}), 7.17-7.30(\mathrm{~m}, 5 \mathrm{H}$, $\left.3-\mathrm{C}_{6} H_{5}\right), 7.40\left(\mathrm{~d}, J=8.9 \mathrm{~Hz}, 2 \mathrm{H}, 2^{\prime}, 6^{\prime}-\mathrm{H}\right), 7.44(\mathrm{t}, J=8.4 \mathrm{~Hz}, 1$ $\mathrm{H}, 6-\mathrm{H}), 7.45$ (s, $1 \mathrm{H}, 1-\mathrm{H}) \mathrm{ppm} .{ }^{13} \mathrm{C}$ NMR: $\delta=36.1$ (C-4), 41.7 (C-3), $55.2\left(8-\mathrm{OCH}_{3}\right), 56.3\left(8-\mathrm{OCH}_{3}\right), 106.2(\mathrm{C}-7), 110.1(\mathrm{C}-5)$, 113.8 (C-3',5'), 114.4 (C-8a), 115.1 (C-1), 117.9 (C-9a), 126.8 (C$\left.2^{\prime}, 6^{\prime}\right), 127.0\left(\mathrm{C}-4^{\prime \prime}\right), 127.3\left(\mathrm{C}-2^{\prime \prime}, 6^{\prime \prime}\right), 128.9\left(\mathrm{C}-3^{\prime \prime}, 5^{\prime \prime}\right), 131.7(\mathrm{C}-$ 1'), 132.9 (C-6), 134.4 (C-2), 140.9 (C-1' $), 157.8$ (C-4b), 159.0 (C$\left.4^{\prime}\right), 159.6$ (C-4a), 160.1 (C-8), 173.9 (C-9) ppm. EI-MS: m/z (\%) = $410\left[\mathrm{M}^{+\cdot}(10), 409\right.$ (28), 408 (100), 407 (34), 391 (21), 390 (15), 380 (18), 379 (62), 363 (26), 362 (43), 347 (16), 305 (9), 153 (10), 152 (13), 137 (14), 121 (21), 99 (16), 78 (9). HRMS (EI): calcd. for $\mathrm{C}_{27} \mathrm{H}_{22} \mathrm{O}_{4}[\mathrm{M}]^{+\cdot} 410.1518$; found 410.1505 .

1-Hydroxy-7-(4-methoxyphenyl)-6-phenyl-9H-xanthen-9-one (120): M.p. $126-128{ }^{\circ} \mathrm{C} .{ }^{1} \mathrm{H}$ NMR: $\delta=3.79$ (s, $\left.3 \mathrm{H}, 4^{\prime}-\mathrm{OCH}_{3}\right), 6.77(\mathrm{~d}, J$ $\left.=8.8 \mathrm{~Hz}, 2 \mathrm{H}, 3^{\prime}, 5^{\prime}-\mathrm{H}\right), 6.81(\mathrm{dd}, J=8.4,0.7 \mathrm{~Hz}, 1 \mathrm{H}, 7-\mathrm{H}), 6.93$ (dd, $J=8.4,0.7 \mathrm{~Hz}, 1 \mathrm{H}, 5-\mathrm{H}), 7.07$ (d, $\left.J=8.8 \mathrm{~Hz}, 2 \mathrm{H}, 2^{\prime}, 6^{\prime}-\mathrm{H}\right)$, $7.18-7.21$ (m, 2 H, $\left.2^{\prime \prime}, 6^{\prime \prime}-\mathrm{H}\right), 7.26-7.30$ (m, 3 H, 3" $\left.3^{\prime \prime} 4^{\prime \prime}, 5^{\prime \prime}-\mathrm{H}\right), 7.49$ (s, $1 \mathrm{H}, 4-\mathrm{H}), 7.59$ (t, $J=8.4 \mathrm{~Hz}, 1 \mathrm{H}, 6-\mathrm{H}), 8.25$ (s, $1 \mathrm{H}, 1-\mathrm{H})$, $12.70(\mathrm{~s}, 1 \mathrm{H}, 8-\mathrm{OH}) \mathrm{ppm} .{ }^{13} \mathrm{C} \mathrm{NMR}: \delta=55.2\left(4^{\prime}-\mathrm{OCH}_{3}\right), 107.0$ (C-5), 109.0 (C-8a), 110.5 (C-7), $113.5\left(\mathrm{C}-3^{\prime}, 5^{\prime}\right), 119.35$ (C-4), 119.38 (C-9a), 127.3 (C-4' ), 127.7 (C-1), 128.2 (C-3"',5' $), 129.5$ $\left(\mathrm{C}-2^{\prime \prime}, 6^{\prime \prime}\right), 131.0\left(\mathrm{C}-2^{\prime}, 6^{\prime}\right), 131.9\left(\mathrm{C}-1^{\prime}\right), 136.7$ (C-6), 136.9 (C-2),

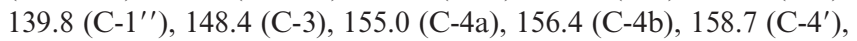
161.9 (C-8) 182.1 (C-9) ppm. EI-MS: $m / z(\%)=394[\mathrm{M}]^{+\cdot}(100)$, 393 (15), 395 (25), 379 (15), 363 (15), 351 (10), 129 (17), 125 (11), 123 (10), 111 (17), 97 (22), 96 (13), 86 (14), 84 (16), 83 (21), 73 (12), 69 (12). HRMS (EI): calcd. for $\mathrm{C}_{27} \mathrm{H}_{22} \mathrm{O}_{4}[\mathrm{M}]^{+\cdot}$ 394.1205; found 394.1204.

7-(3,4-Dimethoxyphenyl)-1-methoxy-6-phenyl-9H-xanthen-9-one (5p): M.p. $189-190{ }^{\circ} \mathrm{C} .{ }^{1} \mathrm{H}$ NMR: $\delta=3.55$ (s, $\left.3 \mathrm{H}, 3^{\prime}-\mathrm{OCH}_{3}\right), 3.87$ (s, $\left.3 \mathrm{H}, 4^{\prime}-\mathrm{OCH}_{3}\right), 4.04$ (s, $\left.3 \mathrm{H}, 8-\mathrm{OCH}_{3}\right), 6.53(\mathrm{~d}, J=1.9 \mathrm{~Hz}, 1 \mathrm{H}$, $\left.2^{\prime}-\mathrm{H}\right), 6.80$ (d, $\left.J=8.3 \mathrm{~Hz}, 1 \mathrm{H}, 5^{\prime}-\mathrm{H}\right), 6.83(\mathrm{dd}, J=8.6,0.9 \mathrm{~Hz}, 1$ $\mathrm{H}, 7-\mathrm{H}), 6.85$ (dd, $\left.J=8.3,1.9 \mathrm{~Hz}, 1 \mathrm{H}, 6^{\prime}-\mathrm{H}\right), 7.09$ (dd, $J=8.6$, $0.9 \mathrm{~Hz}, 1 \mathrm{H}, 5-\mathrm{H}), 7.19-7.22\left(\mathrm{~m}, 2 \mathrm{H}, 2^{\prime \prime}, 6^{\prime \prime}-\mathrm{H}\right), 7.27-7.31(\mathrm{~m}, 3$ $\left.\mathrm{H}, 3^{\prime \prime}, 4^{\prime \prime}, 5^{\prime \prime}-\mathrm{H}\right), 7.47$ (s, $\left.1 \mathrm{H}, 4-\mathrm{H}\right), 7.62$ (t, $\left.J=8.6 \mathrm{~Hz}, 1 \mathrm{H}, 6-\mathrm{H}\right)$, $8.35(\mathrm{~s}, 1 \mathrm{H}, 1-\mathrm{H}) \mathrm{ppm} .{ }^{13} \mathrm{C}$ NMR: $\delta=55.5\left(3^{\prime}-\mathrm{OCH}_{3}\right), 55.8\left(4^{\prime}-\right.$ $\left.\mathrm{OCH}_{3}\right), 56.5\left(8-\mathrm{OCH}_{3}\right), 105.4(\mathrm{C}-7), 110.1$ (C-5), $110.7\left(\mathrm{C}-5^{\prime}\right)$, 112.6 (C-8a), 113.5 (C-2'), 118.8 (C-4), 121.9 (C-9a), 122.1 (C-6'),

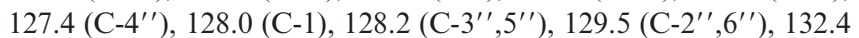
$\left(\mathrm{C}-1^{\prime}\right), 134.8$ (C-6), 136.5 (C-2), 140.3 (C-1' '), 146.9 (C-3), 147.9 and 148.0 (C-3', C-4'), 154.0 (C-4a), 158.2 (C-4b), 160.8 (C-8), 176.4 (C-9) ppm. EI-MS: $m / z=438\left[\right.$ M $^{+*}(100), 424$ (7), 423 (25),
408 (6), 395 (13), 391 (12), 380 (6), 363 (6), 351 (8). $\mathrm{C}_{28} \mathrm{H}_{22} \mathrm{O}_{5}$ (438.47): calcd. for C 76.70, H 5.06; found C 76.73, H 5.27.

7-(3,4-Dimethoxyphenyl)-1-methoxy-6-phenyl-3,4-dihydro-9Hxanthen-9-one (6p): Yellowish oil. ${ }^{1} \mathrm{H}$ NMR: $\delta=2.91$ (dd, $J=17.3$, $\left.1.4 \mathrm{~Hz}, 1 \mathrm{H}, 4_{\text {trans }}-\mathrm{H}\right), 3.60\left(\mathrm{dd}, J=17.3,9.1 \mathrm{~Hz}, 1 \mathrm{H}, 4_{\text {cis }}-\mathrm{H}\right), 3.82$ and $3.84\left(2 \times \mathrm{s}, 6 \mathrm{H}, 3^{\prime}, 4^{\prime}-\mathrm{OCH}_{3}\right), 3.99\left(\mathrm{~s}, 3 \mathrm{H}, 8-\mathrm{OCH}_{3}\right), 4.25$ (dd, $J=9.1,1.4 \mathrm{~Hz}, 1 \mathrm{H}, 3-\mathrm{H}), 6.75\left(\mathrm{~d}, J=8.4 \mathrm{~Hz}, 1 \mathrm{H}, 5^{\prime}-\mathrm{H}\right), 6.78$ (dd, $J=8.4,0.7 \mathrm{~Hz}, 1 \mathrm{H}, 7-\mathrm{H}), 6.90(\mathrm{dd}, J=8.4,0.7 \mathrm{~Hz}, 1 \mathrm{H}, 5-$ $\mathrm{H}), 6.95\left(\mathrm{dd}, J=8.4,2.1 \mathrm{~Hz}, 1 \mathrm{H}, 6^{\prime}-\mathrm{H}\right), 7.07(\mathrm{~d}, J=2.1 \mathrm{~Hz}, 1 \mathrm{H}$, $\left.2^{\prime}-\mathrm{H}\right), 7.18-7.31\left(\mathrm{~m}, 5 \mathrm{H}, 3-\mathrm{C}_{6} H_{5}\right), 7.46(\mathrm{~s}, 1 \mathrm{H}, 1-\mathrm{H}), 7.46$ (t, $J=$ $8.4 \mathrm{~Hz}, 1 \mathrm{H}, 6-\mathrm{H}) \mathrm{ppm} .{ }^{13} \mathrm{C}$ NMR: $\delta=36.1(\mathrm{C}-4), 41.9(\mathrm{C}-3), 55.74$ and $55.84\left(3^{\prime}, 4^{\prime}-\mathrm{OCH}_{3}\right), 56.4\left(8-\mathrm{OCH}_{3}\right), 106.3(\mathrm{C}-7), 108.7\left(\mathrm{C}-2^{\prime}\right)$, 110.1 (C-5), 110.8 (C-5'), 114.5 (C-8a), 115.4 (C-1), 117.9 (C-9a), $118.2\left(\mathrm{C}-6^{\prime}\right), 127.1\left(\mathrm{C}-4^{\prime \prime}\right), 127.3\left(\mathrm{C}-2^{\prime \prime}, 6^{\prime \prime}\right), 128.9\left(\mathrm{C}-3^{\prime \prime}, 5^{\prime \prime}\right), 133.2$ (C-1'), 133.0 (C-6), 135.6 (C-2), $141.1\left(\mathrm{C}-1^{\prime \prime}\right), 148.7$ and 148.8 (C3', C-4'), 157.9 (C-4b), 159.7 (C-4a), 160.2 (C-8), 174.0 (C-9) ppm. EI-MS: $m / z(\%)=440[\mathrm{M}]^{+\cdot}(22), 439$ (24), 408 (100), 423 (20), 395 (10), 391 (9), 363 (8). HRMS (EI): calcd. for $\mathrm{C}_{28} \mathrm{H}_{24} \mathrm{O}_{5}[\mathrm{M}]^{+}$ 440.1624; found 440.1615 .

7-(3,4-Dimethoxyphenyl)-1-hydroxy-6-phenyl-9H-xanthen-9-one (12p): M.p. $186-188^{\circ} \mathrm{C} .{ }^{1} \mathrm{H}$ NMR: $\delta=3.56$ (s, $\left.3 \mathrm{H}, 4^{\prime}-\mathrm{OCH}_{3}\right), 3.88$ $\left(\mathrm{s}, 3 \mathrm{H}, 3^{\prime}-\mathrm{OCH}_{3}\right), 6.52\left(\mathrm{~d}, J=1.9 \mathrm{~Hz}, 1 \mathrm{H}, 2^{\prime}-\mathrm{H}\right), 6.80(\mathrm{~d}, J=$ $\left.8.2 \mathrm{~Hz}, 1 \mathrm{H}, 5^{\prime}-\mathrm{H}\right), 6.82(\mathrm{~d}, J=8.3 \mathrm{~Hz}, 1 \mathrm{H}, 7-\mathrm{H}), 6.84$ (dd, $J=$ 8.2, $\left.1.9 \mathrm{~Hz}, 1 \mathrm{H}, 6^{\prime}-\mathrm{H}\right), 6.95(\mathrm{dd}, J=8.3,0.7 \mathrm{~Hz}, 1 \mathrm{H}, 5-\mathrm{H}), 7.20$ $7.24\left(\mathrm{~m}, 2 \mathrm{H}, 2^{\prime \prime}, 6^{\prime \prime}-\mathrm{H}\right), 7.28-7.32\left(\mathrm{~m}, 3 \mathrm{H}, 3^{\prime \prime}, 4^{\prime \prime}, 5^{\prime \prime}-\mathrm{H}\right), 7.52$ (s, $1 \mathrm{H}, 4-\mathrm{H}), 7.61$ (t, $J=8.3 \mathrm{~Hz}, 1 \mathrm{H}, 6-\mathrm{H}), 8.30$ (s, $1 \mathrm{H}, 1-\mathrm{H}), 12.69$ (s, $1 \mathrm{H}, 8-\mathrm{OH}) \mathrm{ppm} .{ }^{13} \mathrm{C} \mathrm{NMR}: \delta=55.5\left(4^{\prime}-\mathrm{OCH}_{3}\right), 55.7\left(3^{\prime}-\right.$ $\left.\mathrm{OCH}_{3}\right), 107.0$ (C-5), 109.0 (C-8a), 110.5 (C-7), 110.7 (C-5'), 113.3 (C-2'), 119.3 (C-4), 119.4 (C-9a), 122.1 (C-6'), 127.0 (C-1), 127.7 $\left(\mathrm{C}-4^{\prime \prime}\right), 128.2\left(\mathrm{C}-3^{\prime \prime}, 5^{\prime \prime}\right), 129.4\left(\mathrm{C}-2^{\prime \prime}, 6^{\prime \prime}\right), 132.0\left(\mathrm{C}-1^{\prime}\right), 136.7(\mathrm{C}-$ 6), 136.9 (C-2), $139.9\left(\mathrm{C}-1^{\prime \prime}\right), 148.09$ and $148.12\left(\mathrm{C}-3^{\prime}, 4^{\prime}\right), 148.4$ (C-3), 155.0 (C-4a), 156.4 (C-4b), 161.9 (C-8) 182.1 (C-9) ppm. EIMS: $m / z(\%)=424[\mathrm{M}]^{+\cdot}(100), 409(20), 381(10), 377(14), 363$ (10), 349 (13), 337 (10), 139 (10), 137 (14), 125 (12), 111 (16), 110 (13), 97 (19), 96 (13), 86 (13), 84 (19), 83 (15), 81 (16), 73 (12), 69 (17). $\mathrm{C}_{27} \mathrm{H}_{20} \mathrm{O}_{5}$ (424.45): calcd. for $\mathrm{C} 76.40, \mathrm{H} 4.75$; found $\mathrm{C} 76.11$, $\mathrm{H} 4.75$.

6-(3,4-Dimethoxyphenyl)-1-methoxy-7-phenyl-9H-xanthen-9-one (5q): M.p. $188-190{ }^{\circ} \mathrm{C} .{ }^{1} \mathrm{H}$ NMR: $\delta=3.52\left(\mathrm{~s}, 3 \mathrm{H}, 3^{\prime \prime}-\mathrm{OCH}_{3}\right), 3.88$ (s, $\left.3 \mathrm{H}, 4^{\prime \prime}-\mathrm{OCH}_{3}\right), 4.04$ (s, $\left.3 \mathrm{H}, 8-\mathrm{OCH}_{3}\right), 6.54$ (d, $J=2.0 \mathrm{~Hz}, 1$ $\left.\mathrm{H}, 2^{\prime \prime}-\mathrm{H}\right), 6.82\left(\mathrm{~d}, J=8.4 \mathrm{~Hz}, 2 \mathrm{H}, 5^{\prime \prime}-\mathrm{H}, 7-\mathrm{H}\right), 6.90(\mathrm{dd}, J=8.4$, $\left.2.0 \mathrm{~Hz}, 1 \mathrm{H}, 6^{\prime \prime}-\mathrm{H}\right), 7.09$ (dd, $\left.J=8.4,0.8 \mathrm{~Hz}, 1 \mathrm{H}, 5-\mathrm{H}\right), 7.17-7.20$ (m, $\left.2 \mathrm{H}, 2^{\prime}, 6^{\prime}-\mathrm{H}\right), 7.23-7.27$ (m, $\left.3 \mathrm{H}, 3^{\prime}, 4^{\prime}, 5^{\prime}-\mathrm{H}\right), 7.50$ (s, $1 \mathrm{H}, 4-$ $\mathrm{H}), 7.62(\mathrm{t}, J=8.4 \mathrm{~Hz}, 1 \mathrm{H}, 6-\mathrm{H}), 8.31(\mathrm{~s}, 1 \mathrm{H}, 1-\mathrm{H}) \mathrm{ppm} .{ }^{13} \mathrm{C}$ NMR: $\delta=55.5\left(3^{\prime \prime}-\mathrm{OCH}_{3}\right), 55.8\left(4^{\prime \prime}-\mathrm{OCH}_{3}\right), 56.5\left(8-\mathrm{OCH}_{3}\right), 105.5$ (C-7), 110.0 (C-5), 110.7 (C-5"'), 112.6 (C-8a), 113.3 (C-2' $), 118.3$ (C-4), 121.6 (C-9a), 121.8 (C-6' '), 126.7 (C-4'), $128.1\left(\mathrm{C}-3^{\prime}, 5^{\prime}\right)$, 128.4 (C-1), $129.9\left(\mathrm{C}-2^{\prime}, 6^{\prime}\right), 132.3\left(\mathrm{C}-1^{\prime \prime}\right), 134.6(\mathrm{C}-6), 136.8$ (C-2), $140.4\left(\mathrm{C}-1^{\prime}\right), 146.6(\mathrm{C}-3), 148.1$ and $148.5\left(\mathrm{C}-3^{\prime \prime}, \mathrm{C}-4^{\prime \prime}\right), 154.2(\mathrm{C}-$ 4a), 158.2 (C-4b), 160.8 (C-8), 176.3 (C-9) ppm. EI-MS: $m / z(\%)=$ $438 \mathrm{M}^{+\cdot}$ (100), 437 (19), 422 (5), 421 (18), 420 (8), 410 (8), 409 (35), 393 (13), 392 (20), 377 (6), 376 (5), 361 (6), 333 (5). $\mathrm{C}_{28} \mathrm{H}_{22} \mathrm{O}_{5}$ (438.47): calcd. for C 76.70, H 506; found C 76.66, H 5.11.

6-(3,4-Dimethoxyphenyl)-1-methoxy-7-phenyl-7,8-dihydro-9Hxanthen-9-one (6q): Yellowish oil. ${ }^{1} \mathrm{H}$ NMR: $\delta=2.91$ (dd, $J=17.3$, $\left.1.4 \mathrm{~Hz}, 1 \mathrm{H}, 4_{\text {trans }}-\mathrm{H}\right), 3.58\left(\mathrm{dd}, J=17.3,8.6 \mathrm{~Hz}, 1 \mathrm{H}, 4_{\text {cis }}-\mathrm{H}\right), 3.76$ (s, $\left.3 \mathrm{H}, 3^{\prime \prime}-\mathrm{OCH}_{3}\right), 3.80$ (s, $\left.3 \mathrm{H}, 4^{\prime \prime}-\mathrm{OCH}_{3}\right), 3.99$ (s, $\left.3 \mathrm{H}, 8-\mathrm{OCH}_{3}\right)$, $4.22(\mathrm{dd}, J=8.6,1.4 \mathrm{~Hz}, 1 \mathrm{H}, 3-\mathrm{H}), 6.72\left(\mathrm{~d}, J=8.2 \mathrm{~Hz}, 1 \mathrm{H}, 5^{\prime \prime}-\right.$ $\mathrm{H}), 6.79(\mathrm{~d}, J=8.4 \mathrm{~Hz}, 1 \mathrm{H}, 7-\mathrm{H}), 6.81\left(\mathrm{~d}, J=2.0 \mathrm{~Hz}, 1 \mathrm{H}, 2^{\prime \prime}-\right.$ H), $6.84\left(\mathrm{dd}, J=8.2,2.0 \mathrm{~Hz}, 1 \mathrm{H}, 6^{\prime \prime}-\mathrm{H}\right), 6.92(\mathrm{dd}, J=8.4,0.7 \mathrm{~Hz}$, $1 \mathrm{H}, 5-\mathrm{H}), 7.19-7.31$ (m, $\left.3 \mathrm{H}, 3^{\prime}, 4^{\prime}, 5^{\prime}-\mathrm{H}\right), 7.46(\mathrm{~d}, J=8.4 \mathrm{~Hz}, 2 \mathrm{H}$, $\left.2^{\prime}, 6^{\prime}-\mathrm{H}\right), 7.47$ (t, $\left.J=8.4 \mathrm{~Hz}, 1 \mathrm{H}, 6-\mathrm{H}\right), 7.53$ (s, $\left.1 \mathrm{H}, 1-\mathrm{H}\right) \mathrm{ppm}$. 
${ }^{13} \mathrm{C}$ NMR: $\delta=36.5(\mathrm{C}-4), 41.5(\mathrm{C}-3), 55.78$ and $55.82(3,4-$ $\left.\mathrm{OCH}_{3}\right), 56.4\left(8-\mathrm{OCH}_{3}\right), 106.3(\mathrm{C}-7), 110.2(\mathrm{C}-5), 110.3\left(\mathrm{C}-2^{\prime \prime}\right)$, $111.4\left(\mathrm{C}-5^{\prime \prime}\right), 114.4$ (C-8a), 116.9 (C-1), 117.7 (C-9a), 119.3 (C-6"'), $125.6\left(\mathrm{C}-2^{\prime}, 6^{\prime}\right), 127.4\left(\mathrm{C}-4^{\prime}\right), 128.5\left(\mathrm{C}-3^{\prime}, 5^{\prime}\right), 133.1(\mathrm{C}-6), 133.2(\mathrm{C}-$ $\left.1^{\prime \prime}\right), 135.2(\mathrm{C}-2), 139.4\left(\mathrm{C}-1^{\prime}\right), 148.0\left(\mathrm{C}-4^{\prime \prime}\right), 149.1$ (C-3"'), 157.9 (C-4b), 160.2 (C-8), 160.3 (C-4a), 174.0 (C-9) ppm. EI-MS: m/z (\%) $=440[\mathrm{M}]^{+\cdot}$ (18), 439 (23), 438 (100), 437 (23), 421 (24) 420 (11), 410 (10), 409 (42), 393 (18), 392 (25), 361 (8), 151 (8), 137 (9). HRMS (EI): calcd. for $\mathrm{C}_{28} \mathrm{H}_{24} \mathrm{O}_{5}[\mathrm{M}]^{+*}$ 440.1624; found 440.1627 .

6-(3,4-Dimethoxyphenyl)-1-hydroxy-7-phenyl-9H-xanthen-9-one (12q): M.p. $165-167{ }^{\circ} \mathrm{C} .{ }^{1} \mathrm{H}$ NMR: $\delta=3.53$ (s, $3 \mathrm{H}, 3^{\prime \prime}-\mathrm{OCH}_{3}$ ), 3.89 (s, $\left.3 \mathrm{H}, 4^{\prime \prime}-\mathrm{OCH}_{3}\right), 6.55$ (d, $\left.J=2.0 \mathrm{~Hz}, 1 \mathrm{H}, 2^{\prime \prime}-\mathrm{H}\right), 6.83$ (dd, $J=8.4,0.7 \mathrm{~Hz}, 1 \mathrm{H}, 7-\mathrm{H}), 6.84\left(\mathrm{~d}, J=8.3 \mathrm{~Hz}, 1 \mathrm{H}, 5^{\prime \prime}-\mathrm{H}\right), 6.92$ (dd, $\left.J=8.3,2.0 \mathrm{~Hz}, 1 \mathrm{H}, 6^{\prime \prime}-\mathrm{H}\right), 6.97$ (dd, $J=8.4,0.7 \mathrm{~Hz}, 1 \mathrm{H}, 5-$ H), 7.17-7.20 (m, $\left.2 \mathrm{H}, 2^{\prime}, 6^{\prime}-\mathrm{H}\right), 7.25-7.30\left(\mathrm{~m}, 3 \mathrm{H}, 3^{\prime}, 4^{\prime}, 5^{\prime}-\mathrm{H}\right)$, $7.56(\mathrm{~s}, 1 \mathrm{H}, 4-\mathrm{H}), 7.61(\mathrm{t}, J=8.4 \mathrm{~Hz}, 1 \mathrm{H}, 6-\mathrm{H}), 8.28$ (s, $1 \mathrm{H}, 1-$ $\mathrm{H}), 12.71(\mathrm{~s}, 1 \mathrm{H}, 8-\mathrm{OH}) \mathrm{ppm} .{ }^{13} \mathrm{C} \mathrm{NMR}: \delta=55.6\left(3^{\prime \prime}-\mathrm{OCH}_{3}\right)$, $55.8\left(4^{\prime \prime}-\mathrm{OCH}_{3}\right), 107.0(\mathrm{C}-5), 109.1$ (C-8a), 110.6 (C-7), 110.8 (C$\left.5^{\prime \prime}\right), 113.3$ (C-2' ), 118.8 (C-4), 119.2 (C-9a), 1220 (C-6" ), 127.0 (C-4'), 127.6 (C-1), $128.2\left(\mathrm{C}-3^{\prime}, 5^{\prime}\right), 129.8\left(\mathrm{C}-2^{\prime}, 6^{\prime}\right), 132.0\left(\mathrm{C}-1^{\prime \prime}\right)$, 136.7 (C-6), 137.2 (C-2), 140.0 (C-1'), 148.1 (C-3), 148.2 (C-3'), 148.8 (C-4"), 155.4 (C-4a), 156.4 (C-4b), 162.0 (C-8), 182.1 (C9) ppm. EI-MS: $m / z(\%)=424[\mathrm{M}]^{+*}(100), 409$ (12), 393 (12), 377 (11), 350 (8), 349 (13), 337 (8), 84 (10). HRMS (EI): calcd. for $\mathrm{C}_{27} \mathrm{H}_{20} \mathrm{O}_{5}[\mathrm{M}]^{+\cdot}$ 424.1311; found 424.1312.

6,7-Bis(3,4-dimethoxyphenyl)-1-methoxy-9H-xanthen-9-one (5r):

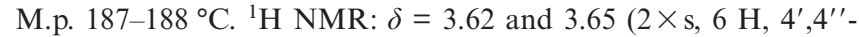
$\left.\mathrm{OCH}_{3}\right), 3.88$ and $3.89\left(2 \times \mathrm{s}, 6 \mathrm{H}, 3^{\prime}, 3^{\prime \prime}-\mathrm{OCH}_{3}\right), 4.04(\mathrm{~s}, 3 \mathrm{H}, 8-$ $\left.\mathrm{OCH}_{3}\right), 6.65-6.66\left(\mathrm{~m}, 2 \mathrm{H}, 2^{\prime}-\mathrm{H}, 2^{\prime \prime}-\mathrm{H}\right), 6.79-6.84(\mathrm{~m}, 5 \mathrm{H}, 7-\mathrm{H}$, $\left.5^{\prime}-\mathrm{H}, 6^{\prime}-\mathrm{H}, 5^{\prime \prime}-\mathrm{H}, 6^{\prime \prime}-\mathrm{H}\right), 7.09$ (dd, $\left.J=8.4,0.7 \mathrm{~Hz}, 1 \mathrm{H}, 5-\mathrm{H}\right), 7.48$ (s, $1 \mathrm{H}, 4-\mathrm{H}), 7.62$ (t, $J=8.4 \mathrm{~Hz}, 1 \mathrm{H}, 6-\mathrm{H}), 8.32$ (s, $1 \mathrm{H}, 1-\mathrm{H}) \mathrm{ppm}$. ${ }^{13} \mathrm{C}$ NMR: $\delta=55.7\left(4^{\prime}, 4^{\prime \prime}-\mathrm{OCH}_{3}\right), 55.8\left(3^{\prime}, 3^{\prime \prime}-\mathrm{OCH}_{3}\right), 56.5(8-$ $\left.\mathrm{OCH}_{3}\right), 105.5(\mathrm{C}-7), 110.0(\mathrm{C}-5), 110.76$ and $110.80\left(\mathrm{C}-5^{\prime}, \mathrm{C}-5^{\prime \prime}\right)$, 112.6 (C-8a), 113.0 and 113.3 (C-2', C-2' '), 118.4 (C-4), 121.7 (C9a), 121.9 and 122.2 (C-6', C-6"'), 128.1 (C-1), 132.7 and 132.9 (C$\left.1^{\prime}, \mathrm{C}-1^{\prime \prime}\right), 134.8$ (C-6), 136.5 (C-2), 146.7 (C-3), 147.9, 148.3 and 148.5 (C-3', C-4', C-3"', C-4"'), 154.0 (C-4a), 158.2 (C-4b), 160.8 (C-8), 176.3 (C-9) ppm. EI-MS: $m / z(\%)=498\left[^{[M}\right]^{+\cdot}(100), 497$ (16), 481 (15), 480 (7), 470 (9), 469 (26), 453 (9), 452 (15), 409 (5), 406 (5), 361 (6), 141 (9), 99 (7), 86 (9), 84 (14), 78 (8). $\mathrm{C}_{30} \mathrm{H}_{26} \mathrm{O}_{7}$ (498.52): calcd. for C 72.28, H 5.26; found C 72.33, H 5.24.

6,7-Bis(3,4-dimethoxyphenyl)-1-methoxy-7,8-dihydro-9H-xanthen-9one (6r): Yellowish oil. ${ }^{1} \mathrm{H}$ NMR: $\delta=2.90(\mathrm{dd}, J=17.3,1.3 \mathrm{~Hz}, 1$ $\left.\mathrm{H}, 4_{\text {trans }}-\mathrm{H}\right), 3.57\left(\mathrm{dd}, J=17.3,8.3 \mathrm{~Hz}, 1 \mathrm{H}, 4_{\text {cis }}-\mathrm{H}\right), 3.76,3.80$, 3.85 and $3.85\left(4 \times \mathrm{s}, 12 \mathrm{H}, 3^{\prime}, 4^{\prime}, 3^{\prime \prime}, 4^{\prime \prime}-\mathrm{OCH}_{3}\right), 3.99(\mathrm{~s}, 3 \mathrm{H}, 8-$ $\left.\mathrm{OCH}_{3}\right), 4.20$ (brd, $\left.J=8.3 \mathrm{~Hz}, 1 \mathrm{H}, 3-\mathrm{H}\right), 6.72(\mathrm{~d}, J=8.2 \mathrm{~Hz}, 1 \mathrm{H}$, $\left.5^{\prime \prime}-\mathrm{H}\right), 6.76\left(\mathrm{~d}, J=8.5 \mathrm{~Hz}, 1 \mathrm{H}, 5^{\prime}-\mathrm{H}\right), 6.79(\mathrm{~d}, J=8.5 \mathrm{~Hz}, 1 \mathrm{H}$, $7-\mathrm{H}), 6.82\left(\mathrm{~d}, J=2.0 \mathrm{~Hz}, 1 \mathrm{H}, 2^{\prime \prime}-\mathrm{H}\right), 6.84(\mathrm{dd}, J=8.2,2.0 \mathrm{~Hz}, 1$ $\left.\mathrm{H}, 6^{\prime \prime}-\mathrm{H}\right), 6.92(\mathrm{dd}, J=8.7,0.7 \mathrm{~Hz}, 1 \mathrm{H}, 5-\mathrm{H}), 6.96(\mathrm{dd}, J=8.5$, $\left.2.1 \mathrm{~Hz}, 1 \mathrm{H}, 6^{\prime}-\mathrm{H}\right), 7.08$ (d, $\left.J=2.1 \mathrm{~Hz}, 1 \mathrm{H}, 2^{\prime}-\mathrm{H}\right), 7.44$ (s, $1 \mathrm{H}, 1-$ $\mathrm{H}), 7.47$ (dd, $J=8.7,8.5 \mathrm{~Hz}, 1 \mathrm{H}, 6-\mathrm{H}) \mathrm{ppm} .{ }^{13} \mathrm{C} \mathrm{NMR}: \delta=36.4$ (C-4), $41.6(\mathrm{C}-3), 55.78,55.82$ and $55.85\left(3^{\prime}, 4^{\prime}, 3^{\prime \prime}, 4^{\prime \prime}-\mathrm{OCH}_{3}\right), 56.4$ $\left(8-\mathrm{OCH}_{3}\right), 106.3(\mathrm{C}-7), 108.8\left(\mathrm{C}-2^{\prime}\right), 110.16$ (C-5), 110.24 (C-2' ), 110.9 (C-5'), 111.4 (C-5' ), 114.4 (C-8a), 115.3 (C-1), 117.8 (C-9a), $118.2\left(\mathrm{C}-6^{\prime}\right), 119.2\left(\mathrm{C}-6^{\prime \prime}\right), 132.3\left(\mathrm{C}-1^{\prime}\right), 133.0(\mathrm{C}-6), 133.5\left(\mathrm{C}-1^{\prime \prime}\right)$, 135.0 (C-2), 148.0, 148.7, 148.8 and 149.1 (C-3', C-4', C-3"', C4" '), 157.9 (C-4b), 159.9 (C-4a), 160.2 (C-8), 174.0 (C-9) ppm. EIMS: $m / z(\%)=500\left[\mathrm{M}^{+\cdot}(21), 499(24), 498(100), 497(12), 481\right.$ (13), 469 (21), 452 (12), 363 (7). HRMS (EI): calcd. for $\mathrm{C}_{30} \mathrm{H}_{28} \mathrm{O}_{7}$ $[\mathrm{M}]^{+\cdot} 500.1835$; found 500.1837 .

6,7-Bis(3,4-dimethoxyphenyl)-1-hydroxy-9H-xanthen-9-one (12r): M.p. $164-166^{\circ} \mathrm{C} .{ }^{1} \mathrm{H}$ NMR: $\delta=3.63$ and $3.66\left(2 \times \mathrm{s}, 6 \mathrm{H}, 4^{\prime}, 4^{\prime \prime}{ }^{\prime}-\right.$
$\left.\mathrm{OCH}_{3}\right), 3.88$ and $3.89\left(2 \times \mathrm{s}, 6 \mathrm{H}, 3,3-\mathrm{OCH}_{3}\right), 6.64-6.66(\mathrm{~m}, 2$ $\left.\mathrm{H}, 2^{\prime}-\mathrm{H}, 2^{\prime \prime}-\mathrm{H}\right), 6.79-6.88$ (m, 5 H, 7-H, 5'-H, 6'-H, 5' $\left.-\mathrm{H}, 6^{\prime \prime}-\mathrm{H}\right)$, $6.94\left(\mathrm{dd}, J=8.4,0.6 \mathrm{~Hz}, 1 \mathrm{H}, 5^{\prime}-\mathrm{H}\right), 7.52$ (s, $\left.1 \mathrm{H}, 4-\mathrm{H}\right), 7.60$ (t, $J$ $=8.4 \mathrm{~Hz}, 1 \mathrm{H}, 6-\mathrm{H}), 8.26(\mathrm{~s}, 1 \mathrm{H}, 1-\mathrm{H}), 12.70(\mathrm{~s}, 1 \mathrm{H}, 8-\mathrm{OH}) \mathrm{ppm}$. ${ }^{13} \mathrm{C}$ NMR: $\delta=55.68$ and $55.71\left(4^{\prime}, 4^{\prime \prime}-\mathrm{OCH}_{3}\right), 55.8\left(3^{\prime}, 3^{\prime \prime}-\mathrm{OCH}_{3}\right)$, 107.0 (C-5), 109.0 (C-8a), 110.5 (C-7), 110.77 and 110.81 (C-5', C$\left.5^{\prime \prime}\right), 112.9$ and 113.1 (C-2', C-2' '), 118.4 (C-4), 119.1 (C-9a), 121.9 and 122.1 (C-6', C-6" '), 127.1 (C-1), 132.2 and 132.4 (C-1', C-1' '), 136.7 (C-6), 136.9 (C-2), 148.11, 148.13, 148.36, 148.38 and 148.7 (C-3, C-3', C-4', C-3'", C-4"'), 155.1 (C-4a), 156.3 (C-4b), 161.9 (C-8), 182.0 (C-9) ppm. EI-MS: $m / z(\%)=484[\mathrm{M}]^{+\cdot}(100), 469$ (9), 185 (12), 129 (19), 111 (10), 97 (16), 83 (13), 73 (20), 71 (10), 69 (13), 60 (12). HRMS (EI): calcd. for $\mathrm{C}_{29} \mathrm{H}_{24} \mathrm{O}_{7}[\mathrm{M}]^{+*} 484.1522$; found 484.1523 .

1,3-Dimethoxy-6,7-diphenyl-9H-xanthen-9-one (5s): M.p. 209$210{ }^{\circ} \mathrm{C} .{ }^{1} \mathrm{H}$ NMR: $\delta=3.93$ (s, $\left.3 \mathrm{H}, 6-\mathrm{OCH}_{3}\right), 4.00$ (s, $3 \mathrm{H}, 8-\mathrm{OCH}_{3}$ ), $6.37(\mathrm{~d}, J=2.3 \mathrm{~Hz}, 1 \mathrm{H}, 7-\mathrm{H}), 6.53(\mathrm{~d}, J=2.3 \mathrm{~Hz}, 1 \mathrm{H}, 5-\mathrm{H}), 7.14$ $7.27\left(\mathrm{~m}, 10 \mathrm{H}, 2,3-\mathrm{C}_{6} H_{5}\right), 7.43(\mathrm{~s}, 1 \mathrm{H}, 4-\mathrm{H}), 8.32$ (s, $\left.1 \mathrm{H}, 1-\mathrm{H}\right) \mathrm{ppm}$. ${ }^{13} \mathrm{C}$ NMR: $\delta=55.8\left(6-\mathrm{OCH}_{3}\right), 56.4\left(8-\mathrm{OCH}_{3}\right), 92.9(\mathrm{C}-5), 95.2(\mathrm{C}-$ 7), 107.4 (C-8a), 118.4 (C-4), 122.0 (C-9a), 126.7 (C-4'), 127.4 (C$\left.4^{\prime \prime}\right), 128.0\left(\mathrm{C}-3^{\prime}, 5^{\prime}\right), 128.1\left(\mathrm{C}-3^{\prime \prime}, 5^{\prime \prime}\right), 128.4(\mathrm{C}-1), 129.6\left(\mathrm{C}-2^{\prime \prime}, 6^{\prime \prime}\right)$, $130.0\left(\mathrm{C}-2^{\prime}, 6^{\prime}\right), 136.8(\mathrm{C}-2), 140.05$ and 140.08 (C-1', C-1' $\left.{ }^{\prime \prime}\right), 146.4$ (C-3), 154.1 (C-4a), 159.9 (C-4b), 162.0 (C-8), 164.9 (C-6), 175.2 (C-9) ppm. EI-MS: $m / z(\%)=408[\mathrm{M}]^{+\cdot}(100), 407$ (40), 394 (5), 391 (13), 390 (8), 380 (14), 379 (66), 378 (15), 377 (39), 363 (13), 362 (27), 336 (7), 334 (14), 333 (13), 276 (6), 226 (5), 215 (8), 189 (5). $\mathrm{C}_{27} \mathrm{H}_{20} \mathrm{O}_{4}$ (408.45): calcd. for C 79.40, H 4.94; found C 79.22, H 5.00 .

1,3-Dimethoxy-6,7-diphenyl-7,8-dihydro-9H-xanthen-9-one (6s): M.p. $189-191{ }^{\circ} \mathrm{C} .{ }^{1} \mathrm{H}$ NMR: $\delta=2.90(\mathrm{dd}, J=17.2,1.4 \mathrm{~Hz}, 1 \mathrm{H}$, $\left.4_{\text {trans }}-\mathrm{H}\right), 3.61\left(\mathrm{dd}, J=17.2,8.9 \mathrm{~Hz}, 1 \mathrm{H}, 4_{\text {cis }}-\mathrm{H}\right), 3.86(\mathrm{~s}, 3 \mathrm{H}, 6-$ $\left.\mathrm{OCH}_{3}\right), 3.98\left(\mathrm{~s}, 3 \mathrm{H}, 8-\mathrm{OCH}_{3}\right), 4.28($ brd, $J=8.9 \mathrm{~Hz}, 1 \mathrm{H}, 3-\mathrm{H})$, 6.36 and $6.37(\mathrm{AB}, J=2.6 \mathrm{~Hz}, 2 \mathrm{H}, 5-\mathrm{H}, 7-\mathrm{H}), 7.21-7.33(\mathrm{~m}, 8 \mathrm{H}$, $\left.3^{\prime}, 4^{\prime}, 5^{\prime}-\mathrm{H}, 3-\mathrm{C}_{6} H_{5}\right), 7.46-7.49\left(\mathrm{~m}, 2 \mathrm{H}, 2^{\prime}, 6^{\prime}-\mathrm{H}\right), 7.56(\mathrm{~s}, 1 \mathrm{H}, 1-$ H) ppm. ${ }^{13} \mathrm{C}$ NMR: $\delta=36.2(\mathrm{C}-4), 41.8(\mathrm{C}-3), 55.6\left(6-\mathrm{OCH}_{3}\right), 56.3$ (8-OCH $\mathrm{OH}_{3}$ ), 92.8 (C-5), 96.0 (C-7), 109.1 (C-8a), 117.2 (C-1), 117.6 (C-9a), $125.6\left(\mathrm{C}-2^{\prime}, 6^{\prime}\right), 127.1$ and $127.3\left(\mathrm{C}-4^{\prime}, \mathrm{C}-4^{\prime \prime}\right), 127.4(\mathrm{C}-$ $\left.2^{\prime \prime}, 6^{\prime \prime}\right), 128.4\left(\mathrm{C}-3^{\prime}, 5^{\prime}\right), 128.9\left(\mathrm{C}-3^{\prime \prime}, 5^{\prime \prime}\right), 134.6(\mathrm{C}-2), 139.3\left(\mathrm{C}-1^{\prime}\right)$, $140.9\left(\mathrm{C}-1^{\prime \prime}\right), 159.39$ and 159.43 (C-4a, C-4b), 161.3 (C-8), 163.4 (C-6), 173.3 (C-9) ppm. EI-MS: $m / z(\%)=410[\mathrm{M}]^{+\cdot}(17), 409$ (29), 408 (100), 407 (45), 395 (15), 394 (45), 393 (23), 391 (20), 390 (10), 380 (23), 379 (82), 378 (24), 377 (49), 365 (13), 364 (12), 363 (21), 362 (32), 334 (19), 333 (20), 289 (11), 228 (11), 215 (12), 105 (11). HRMS (EI): calcd. for $\mathrm{C}_{27} \mathrm{H}_{22} \mathrm{O}_{4}[\mathrm{M}]^{+\cdot} 410.1518$; found 410.1510 .

1-Hydroxy-3-methoxy-6,7-diphenyl-9H-xanthen-9-one (12s): M.p. 197-200 ${ }^{\circ} \mathrm{C} .{ }^{1} \mathrm{H}$ NMR: $\delta=3.91\left(\mathrm{~s}, 3 \mathrm{H}, 6-\mathrm{OCH}_{3}\right), 6.38(\mathrm{~d}, J=$ $2.2 \mathrm{~Hz}, 1 \mathrm{H}, 7-\mathrm{H}), 6.46(\mathrm{~d}, J=2.2 \mathrm{~Hz}, 1 \mathrm{H}, 5-\mathrm{H}), 7.14-7.20$ (m, 4 $\left.\mathrm{H}, 2^{\prime}, 6^{\prime}-\mathrm{H}, 2^{\prime \prime}, 6^{\prime \prime}-\mathrm{H}\right), 7.22-7.29$ (m, $\left.6 \mathrm{H}, 3^{\prime}, 4^{\prime}, 5^{\prime}-\mathrm{H}, 3^{\prime \prime}, 4^{\prime \prime}, 5^{\prime \prime}-\mathrm{H}\right)$, 7.49 (s, $1 \mathrm{H}, 4-\mathrm{H}), 8.27$ (s, $1 \mathrm{H}, 1-\mathrm{H}), 12.91$ (s, $1 \mathrm{H}, 8-\mathrm{OH}) \mathrm{ppm}$. ${ }^{13} \mathrm{C}$ NMR: $\delta=55.8\left(6-\mathrm{OCH}_{3}\right), 93.0(\mathrm{C}-5), 97.1$ (C-7), 104.0 (C-8a), 119.1 (C-4), 119.5 (C-9a), 127.0 and 127.7 (C-4', C-4' '), 127.5 (C1), 128.0 and $128.2\left(\mathrm{C}-3^{\prime}, 5^{\prime}, \mathrm{C}-3^{\prime \prime}, 5^{\prime \prime}\right), 129.6$ and $129.9\left(\mathrm{C}-2^{\prime}, 6^{\prime}\right.$, $\left.\mathrm{C}-2^{\prime \prime}, 6^{\prime \prime}\right), 137.2(\mathrm{C}-2), 139.67$ and $139.74\left(\mathrm{C}-1^{\prime}, \mathrm{C}-1^{\prime \prime}\right), 147.8(\mathrm{C}-$ 3), 155.1 (C-4a), 157.8 (C-4b), 163.6 (C-8), 166.7 (C-6), 180.6 (C9) ppm. EI-MS: $m / z(\%)=394[\mathrm{M}]^{+\cdot}(100), 393(31), 365(16), 350$ (6). HRMS (EI): calcd. for $\mathrm{C}_{26} \mathrm{H}_{18} \mathrm{O}_{4}[\mathrm{M}]^{+\cdot} 394.1205$; found 394.1209 .

Synthesis of the Hydroxylated 2,3-Diaryl-9H-xanthen-9-ones 13b-s: A solution of the appropriate 2,3-diaryl-9H-xanthen-9-one (5b-s) in recently distilled dichloromethane $(3 \mathrm{~mL})$ was cooled to $-78^{\circ} \mathrm{C}$ and placed under nitrogen. A solution of boron tribromide in dichloromethane $(0.1 \mathrm{M}, 2.5$ equiv. for each methyl group to be 
cleaved) was gradually added. The reaction mixture was stirred and left at room temperature for a period of time according to the substitution of the compounds (one hour for each group to be cleaved). After that period, the solution was poured into water $(20 \mathrm{~mL})$ and vigorously stirred until the formation of a yellow precipitate. The solid was washed abundantly with water $(4 \times 50 \mathrm{~mL})$ and then with light petroleum $(4 \times 20 \mathrm{~mL})$ to afford the hydroxylated 2,3-diaryl9H-xanthen-9-one (13b-s) in good yields.

2-(4-Hydroxyphenyl)-3-phenyl-9H-xanthen-9-one (13b): Reaction time $1 \mathrm{~h}, 72 \%$ yield; m.p. $241-242{ }^{\circ} \mathrm{C} .{ }^{1} \mathrm{H}$ NMR ([D $]$ DMSO): $\delta=$ $6.66\left(\mathrm{~d}, J=8.5 \mathrm{~Hz}, 2 \mathrm{H}, 3^{\prime}, 5^{\prime}-\mathrm{H}\right), 6.95\left(\mathrm{~d}, J=8.5 \mathrm{~Hz}, 2 \mathrm{H}, 2^{\prime}, 6^{\prime}-\right.$ H), 7.22-7.25 (m, 2 H, $\left.2^{\prime \prime}, 6^{\prime \prime}-\mathrm{H}\right), 7.32-7.34$ (m, $\left.3 \mathrm{H}, 3^{\prime \prime}, 4^{\prime \prime}, 5^{\prime \prime}-\mathrm{H}\right)$, $7.52(\mathrm{t}, J=7.7 \mathrm{~Hz}, 1 \mathrm{H}, 7-\mathrm{H}), 7.64(\mathrm{~s}, 1 \mathrm{H}, 4-\mathrm{H}), 7.69(\mathrm{~d}, J=$ $8.1 \mathrm{~Hz}, 1 \mathrm{H}, 5-\mathrm{H}), 7.91$ (ddd, $J=8.1,7.7,1.6 \mathrm{~Hz}, 1 \mathrm{H}, 6-\mathrm{H}), 8.06$ (s, $1 \mathrm{H}, 1-\mathrm{H}), 8.23$ (dd, $J=7.7,1.6 \mathrm{~Hz}, 1 \mathrm{H}, 8-\mathrm{H}), 9.53$ (s, $1 \mathrm{H}, 4^{\prime}-$ $\mathrm{OH}) \mathrm{ppm} .{ }^{13} \mathrm{C}$ NMR ([D $\left.] \mathrm{DMSO}\right): \delta=115.1\left(\mathrm{C}-3^{\prime}, 5^{\prime}\right), 118.3(\mathrm{C}-$ 5), 119.5 (C-4), 120.1 (C-9a), 121.2 (C-8a), 124.5 (C-7), 126.1 (C8), 127.0 (C-1), $127.7\left(\mathrm{C}-4^{\prime \prime}\right), 128.3\left(\mathrm{C}-3^{\prime \prime}, 5^{\prime \prime}\right), 129.4\left(\mathrm{C}-2^{\prime \prime}, 6^{\prime \prime}\right)$, $130.0\left(\mathrm{C}-1^{\prime}\right), 130.7\left(\mathrm{C}-2^{\prime}, 6^{\prime}\right), 135.6(\mathrm{C}-6), 136.7$ (C-2), $139.5(\mathrm{C}-$ $\left.1^{\prime \prime}\right), 147.2$ (C-3), 154.4 (C-4a), 155.8 (C-4b), 156.5 (C-4'), 175.8 (C9) $\mathrm{ppm}$. EI-MS: $m / z(\%)=364[\mathrm{M}]^{+\cdot}(100), 349$ (3), 335 (4), 305 (5), 276 (3), 244 (3), 213 (3), 173 (4), 138 (2), 77 (2). HRMS (EI): calcd. for $\mathrm{C}_{25} \mathrm{H}_{16} \mathrm{O}_{3}[\mathrm{M}]^{+\cdot} 364.1099$; found 364.1100 .

2-(3,4-Dihydroxyphenyl)-3-phenyl-9H-xanthen-9-one (13c): Reaction time $2 \mathrm{~h}, 80 \%$ yield; m.p. $171-173{ }^{\circ} \mathrm{C} .{ }^{1} \mathrm{H}$ NMR ([D $]$ DMSO): $\delta=6.37\left(\mathrm{dd}, J=8.1,2.0 \mathrm{~Hz}, 1 \mathrm{H}, 6^{\prime}-\mathrm{H}\right), 6.58(\mathrm{~d}, J=2.0 \mathrm{~Hz}, 1 \mathrm{H}$, $\left.2^{\prime}-\mathrm{H}\right), 6.63\left(\mathrm{~d}, J=8.1 \mathrm{~Hz}, 1 \mathrm{H}, 5^{\prime}-\mathrm{H}\right), 7.24-7.30$ (m, $2 \mathrm{H}, 2^{\prime \prime}, 6^{\prime \prime}$ H), 7.33-7.36 (m, $\left.3 \mathrm{H}, 3^{\prime \prime}, 4^{\prime \prime}, 5^{\prime \prime}-\mathrm{H}\right), 7.50(\mathrm{dt}, J=7.7,0.5 \mathrm{~Hz}, 1$ $\mathrm{H}, 7-\mathrm{H}), 7.60(\mathrm{~s}, 1 \mathrm{H}, 4-\mathrm{H}), 7.67(\mathrm{~d}, J=8.2 \mathrm{~Hz}, 1 \mathrm{H}, 5-\mathrm{H}), 7.89$ (ddd, $J=8.2,7.7,1.4 \mathrm{~Hz}, 1 \mathrm{H}, 6-\mathrm{H}), 8.03$ (s, $1 \mathrm{H}, 1-\mathrm{H}), 8.22$ (dd, $J=7.7,1.4 \mathrm{~Hz}, 1 \mathrm{H}, 8-\mathrm{H}), 8.94$ and $8.99\left(2 \times\right.$ brs, $2 \mathrm{H}, 3^{\prime}, 4^{\prime}-$ $\mathrm{O} H)$ ppm. ${ }^{13} \mathrm{C}$ NMR ([D $]$ DMSO): $\delta=115.5\left(\mathrm{C}-5^{\prime}\right), 117.0\left(\mathrm{C}-2^{\prime}\right)$, 118.3 (C-5), 119.4 (C-4), 120.0 (C-9a), 120.8 (C-6'), 121.2 (C-8a), 124.5 (C-7), 126.1 (C-8), 127.0 (C-1), 127.7 (C-4"'), 128.2 (C$\left.3^{\prime \prime}, 5^{\prime \prime}\right), 129.3\left(\mathrm{C}-2^{\prime \prime}, 6^{\prime \prime}\right), 130.7\left(\mathrm{C}-1^{\prime}\right), 135.6(\mathrm{C}-6), 136.9(\mathrm{C}-2)$, 139.6 (C-1' $), 144.6\left(\mathrm{C}-3^{\prime}\right), 145.0$ (C-4'), 147.0 (C-3), 154.3 (C-4a), 155.8 (C-4b), 175.8 (C-9) ppm. EI-MS: $m / z(\%)=380[\mathrm{M}]^{+\cdot}(100)$, 363 (5), 333 (12), 321 (3), 305 (6), 292 (2), 276 (4), 263 (2), 239 (2), 213 (2), 181 (2), 167 (8), 152 (2), 138 (4), 121 (2), 77 (2). HRMS (EI): calcd. for $\mathrm{C}_{25} \mathrm{H}_{16} \mathrm{O}_{4}[\mathrm{M}]^{+\cdot} 380.1049$; found 380.1035 .

3-(4-Hydroxyphenyl)-2-phenyl-9H-xanthen-9-one (13d, 13g): Reaction time $24 \mathrm{~h}, 69 \%$ yield when obtained from compound $\mathbf{5 d} ; 1 \mathrm{~h}$, $82 \%$ when obtained from compound 5g; m.p. $244-245^{\circ} \mathrm{C} .{ }^{1} \mathrm{H}$ NMR (500 MHz, [D 6 ]DMSO): $\delta=6.69$ (d, $J=8.6 \mathrm{~Hz}, 2 \mathrm{H}, 3^{\prime \prime}, 5^{\prime \prime}$ H), $7.05\left(\mathrm{~d}, J=8.6 \mathrm{~Hz}, 2 \mathrm{H}, 2^{\prime \prime}, 6^{\prime \prime}-\mathrm{H}\right), 7.19(\mathrm{dd}, J=7.8,1.5 \mathrm{~Hz}$, $2 \mathrm{H}, 2^{\prime}, 6^{\prime}-\mathrm{H}$ ), 7.30-7.35 (m, $\left.3 \mathrm{H}, 3^{\prime}, 4^{\prime}, 5^{\prime}-\mathrm{H}\right), 7.53$ (ddd, $J=7.7$, 7.6, $0.9 \mathrm{~Hz}, 1 \mathrm{H}, 7-\mathrm{H}), 7.65(\mathrm{~s}, 1 \mathrm{H}, 4-\mathrm{H}), 7.71(\mathrm{~d}, J=8.1 \mathrm{~Hz}, 1 \mathrm{H}$, 5-H), 7.92 (ddd, $J=8.1,7.6,1.6 \mathrm{~Hz}, 1 \mathrm{H}, 6-\mathrm{H}), 8.08$ (s, $1 \mathrm{H}, 1-\mathrm{H})$, $8.24(\mathrm{dd}, J=7.7,1.6 \mathrm{~Hz}, 1 \mathrm{H}, 8-\mathrm{H}), 9.67\left(\mathrm{~s}, 1 \mathrm{H}, 4^{\prime \prime}-\mathrm{OH}\right) \mathrm{ppm}$. ${ }^{13} \mathrm{C}$ NMR (500 MHz, [D $]$ DMSO): $\delta=115.1$ (C-3" , 5" $\left.^{\prime \prime}\right), 118.3$ (C5), 119.1 (C-4), 119.6 (C-9a), 121.3 (C-8a), 124.5 (C-7), 126.1 (C8), $127.0\left(\mathrm{C}-4^{\prime}\right), 127.4(\mathrm{C}-1), 128.3\left(\mathrm{C}-3^{\prime}, 5^{\prime}\right), 129.5\left(\mathrm{C}-2^{\prime}, 6^{\prime}\right), 129.7$ $\left(\mathrm{C}-1^{\prime \prime}\right), 130.8\left(\mathrm{C}-2^{\prime \prime}, 6^{\prime \prime}\right), 135.6(\mathrm{C}-6), 136.5(\mathrm{C}-2), 139.9\left(\mathrm{C}-1^{\prime}\right)$, 147.4 (C-3), 154.8 (C-4a), 155.8 (C-4b), 157.2 (C-4" '), 175.7 (C9) $\mathrm{ppm}$. EI-MS: $m / z(\%)=364[\mathrm{M}]^{+\cdot}(100), 347$ (7), 335 (4), 305 (6), 289 (4), 276 (3), 244 (4), 213 (4), 173 (5), 138 (2), 84 (4), 66 (4). $\mathrm{C}_{25} \mathrm{H}_{16} \mathrm{O}_{3}$ (364.39): calcd. for $\mathrm{C} 82.40, \mathrm{H} 4.43$; found $\mathrm{C} 82.50, \mathrm{H}$ 4.43 .

2,3-Bis(4-hydroxyphenyl)-9H-xanthen-9-one (13e, 13h): Reaction time $24 \mathrm{~h}, 82 \%$ yield when obtained from compound $5 \mathrm{e} ; 2 \mathrm{~h}, 94 \%$ when obtained from compound $\mathbf{5 h}$; m.p. $231-233{ }^{\circ} \mathrm{C} .{ }^{1} \mathrm{H}$ NMR (500 MHz, [D 6 ]DMSO): $\delta=6.68$ (d, $\left.J=8.7 \mathrm{~Hz}, 2 \mathrm{H}, 3^{\prime}, 5^{\prime}-\mathrm{H}\right), 6.69$ (d, $\left.J=8.6 \mathrm{~Hz}, 2 \mathrm{H}, 3^{\prime \prime}, 5^{\prime \prime}-\mathrm{H}\right), 6.94\left(\mathrm{~d}, J=8.7 \mathrm{~Hz}, 2 \mathrm{H}, 2^{\prime}, 6^{\prime}-\mathrm{H}\right)$, 7.02 (d, $\left.J=8.6 \mathrm{~Hz}, 2 \mathrm{H}, 2^{\prime \prime}, 6^{\prime \prime}-\mathrm{H}\right), 7.47$ (t, $\left.J=7.8 \mathrm{~Hz}, 1 \mathrm{H}, 7-\mathrm{H}\right)$, $7.51(\mathrm{~s}, 1 \mathrm{H}, 4-\mathrm{H}), 7.63(\mathrm{~d}, J=8.0 \mathrm{~Hz}, 1 \mathrm{H}, 5-\mathrm{H}), 7.87$ (ddd, $J=$ 8.0, 7.8, 1.6 Hz, $1 \mathrm{H}, 6-\mathrm{H}), 7.98$ (s, $1 \mathrm{H}, 1-\mathrm{H}), 8.19$ (dd, $J=7.8$, $1.6 \mathrm{~Hz}, 1 \mathrm{H}, 8-\mathrm{H}), 9.51$ and $9.64\left(2 \times \mathrm{s}, 2 \mathrm{H}, 4^{\prime}, 4^{\prime \prime}-\mathrm{OH}\right) \mathrm{ppm} .{ }^{13} \mathrm{C}$ NMR (500 MHz, [D $]$ DMSO): $\delta=115.1\left(\mathrm{C}-3^{\prime}, 5^{\prime}, \mathrm{C}-3^{\prime \prime}, 5^{\prime \prime}\right), 118.2$ (C-5), 118.9 (C-4), 119.5 (C-9a), 121.2 (C-8a), 124.4 (C-7), 126.0 (C-8), 127.0 (C-1), $130.0\left(\mathrm{C}-1^{\prime \prime}\right), 130.4\left(\mathrm{C}-1^{\prime}\right), 130.6\left(\mathrm{C}-2^{\prime}, 6^{\prime}\right), 130.7$ (C-2' ',6" ), 135.6 (C-6), 136.6 (C-2), 147.3 (C-3), 154.4 (C-4a), 155.7 (C-4b), 156.4 (C-4'), 157.1 (C-4' ), 175.7 (C-9) ppm. EI-MS: $\mathrm{m} / \mathrm{z}(\%)=380[\mathrm{M}]^{+\cdot}(43), 363(2), 321(1), 305(1), 264$ (2), $236(3)$, 174 (100), 158 (70), 144 (28), 131 (48), 121 (30), 115 (26), 105 (54), 91 (27), 77 (64), 65 (20), 53 (22), 51 (24). $\mathrm{C}_{25} \mathrm{H}_{16} \mathrm{O}_{4}$ (380.39): calcd. for C 78.94, H 4.24; found C 78.95, H 4.23.

3-(4-Hydroxyphenyl)-2-(3,4-dihydroxyphenyl)-9H-xanthen-9-one (13f, 13i): Reaction time $24 \mathrm{~h}, 92 \%$ yield when obtained from compound $\mathbf{5 f}, 3 \mathrm{~h}, 80 \%$ when obtained from compound 5i; m.p. 260 $261{ }^{\circ} \mathrm{C} .{ }^{1} \mathrm{H}$ NMR $\left(\left[\mathrm{D}_{6}\right] \mathrm{DMSO}\right): \delta=6.41(\mathrm{dd}, J=8.1,2.1 \mathrm{~Hz}, 1 \mathrm{H}$, $\left.6^{\prime}-\mathrm{H}\right), 6.58\left(\mathrm{~d}, J=2.1 \mathrm{~Hz}, 1 \mathrm{H}, 2^{\prime}-\mathrm{H}\right), 6.65\left(\mathrm{~d}, J=8.1 \mathrm{~Hz}, 1 \mathrm{H}, 5^{\prime}-\right.$ $\mathrm{H}), 6.71\left(\mathrm{~d}, J=8.6 \mathrm{~Hz}, 2 \mathrm{H}, 3^{\prime \prime}, 5^{\prime \prime}-\mathrm{H}\right), 7.08(\mathrm{~d}, J=8.6 \mathrm{~Hz}, 2 \mathrm{H}$, $\left.2^{\prime \prime}, 6^{\prime \prime}-\mathrm{H}\right), 7.51(\mathrm{dd}, J=7.7,7.4 \mathrm{~Hz}, 1 \mathrm{H}, 7-\mathrm{H}), 7.58$ (s, $\left.1 \mathrm{H}, 4-\mathrm{H}\right)$, $7.70(\mathrm{~d}, J=7.9 \mathrm{~Hz}, 1 \mathrm{H}, 5-\mathrm{H}), 7.90(\mathrm{ddd}, J=7.9,7.4,1.6 \mathrm{~Hz}, 1$ H, 6-H), 8.00 (s, $1 \mathrm{H}, 1-\mathrm{H}), 8.23$ (dd, $J=7.7,1.6 \mathrm{~Hz}, 1 \mathrm{H}, 8-\mathrm{H})$, $8.95\left(\mathrm{~s}, 1 \mathrm{H}, 4^{\prime}-\mathrm{OH}\right), 9.01\left(\mathrm{~s}, 1 \mathrm{H}, 3^{\prime}-\mathrm{OH}\right), 9.65$ (s, $1 \mathrm{H}, 4^{\prime \prime}$ $\mathrm{O} H)$ ppm. ${ }^{13} \mathrm{C}$ NMR ([D $]$ DMSO): $\delta=115.1\left(\mathrm{C}-3^{\prime \prime}, 5^{\prime \prime}\right), 115.5(\mathrm{C}-$ 5'), 116.9 (C-2'), 118.3 (C-5), 118.9 (C-4), 119.5 (C-9a), 120.6 (C$\left.6^{\prime}\right), 121.3$ (C-8a), 124.4 (C-7), 126.1 (C-8), 127.0 (C-1), 130.0 (C$\left.1^{\prime \prime}\right), 130.6\left(\mathrm{C}-2^{\prime \prime}, 6^{\prime \prime}\right), 131.1\left(\mathrm{C}-1^{\prime}\right), 135.6$ (C-6), 136.8 (C-2), 144.5 (C-4'), 145.0 (C-3'), 147.3 (C-3), 154.4 (C-4a), 155.8 (C-4b), 157.2 $\left(\mathrm{C}-4^{\prime \prime}\right), 175.8$ (C-9) ppm. EI-MS: $m / z(\%)=396[\mathrm{M}]^{+\cdot}(100), 380$ (7), 349 (7), 175 (5), 105 (1), 78 (15), 77 (5), 63 (21). $\mathrm{C}_{25} \mathrm{H}_{16} \mathrm{O}_{5}$ (396.39): calcd. for C 75.75, H 4.07; found C 75.34, H 4.06.

3-(3,4-Dihydroxyphenyl)-2-phenyl-9H-xanthen-9-one (13j): Reaction time $2 \mathrm{~h}, 80 \%$ yield; m.p. $244-246{ }^{\circ} \mathrm{C} .{ }^{1} \mathrm{H}$ NMR ([D $]$ DMSO): $\delta=$ $6.48\left(\mathrm{dd}, J=8.1,1.9 \mathrm{~Hz}, 1 \mathrm{H}, 6^{\prime \prime}-\mathrm{H}\right), 6.62\left(\mathrm{~d}, J=1.9 \mathrm{~Hz}, 1 \mathrm{H}, 2^{\prime \prime}\right.$ $\mathrm{H}), 6.65\left(\mathrm{~d}, J=8.1 \mathrm{~Hz}, 1 \mathrm{H}, 5^{\prime \prime}-\mathrm{H}\right), 7.18-7.21\left(\mathrm{~m}, 2 \mathrm{H}, 2^{\prime}, 6^{\prime}-\mathrm{H}\right)$, $7.28-7.35\left(\mathrm{~m}, 3 \mathrm{H}, 3^{\prime}, 4^{\prime}, 5^{\prime}-\mathrm{H}\right), 7.51(\mathrm{dd}, J=7.9,7.5 \mathrm{~Hz}, 1 \mathrm{H}, 7-$ H), 7.55 (s, $1 \mathrm{H}, 4-\mathrm{H}), 7.68$ (d, $J=8.1 \mathrm{~Hz}, 1 \mathrm{H}, 5-\mathrm{H}), 7.90$ (ddd, $J$ $=8.1,7.5,1.0 \mathrm{~Hz}, 1 \mathrm{H}, 6-\mathrm{H}), 8.05(\mathrm{~s}, 1 \mathrm{H}, 1-\mathrm{H}), 8.22(\mathrm{dd}, J=7.9$, $1.1 \mathrm{~Hz}, 1 \mathrm{H}, 8-\mathrm{H}), 8.97$ (s, $\left.1 \mathrm{H}, 4^{\prime \prime}-\mathrm{O} H\right), 9.16$ (s, $\left.1 \mathrm{H}, 3^{\prime \prime}{ }^{\prime}-\mathrm{O} H\right) \mathrm{ppm}$. ${ }^{13} \mathrm{C}$ NMR ([D $]$ DMSO): $\delta=115.4\left(\mathrm{C}-5^{\prime \prime}\right), 116.9\left(\mathrm{C}-2^{\prime \prime}\right), 118.3(\mathrm{C}-$ 5), 119.0 (C-4), 119.6 (C-9a), 120.9 (C-6"'), 121.3 (C-8a), 124.5 (C7), 126.1 (C-8), 127.0 (C-4'), 127.4 (C-1), 128.3 (C-3', 5'), 129.4 (C$\left.2^{\prime}, 6^{\prime}\right), 130.3$ (C-1' $), 135.6$ (C-6), 136.5 (C-2), 140.0 (C-1'), 145.0 (C-3'), 145.4 (C-4"'), 147.7 (C-3), 154.7 (C-4a), 155.8 (C-4b), 175.7 (C-9) ppm. EI-MS: $m / z(\%)=380[\mathrm{M}]^{+*}(100), 379$ (17), 363 (16), 334 (12), 333 (15), 167 (13), 129 (17), 115 (11), 111 (16), 105 (17), 97 (23), 96 (12), 95 (16), 85 (16), 84 (48), 83 (27), 77 (12), 73 (18), 71 (21), 69 (31), 66 (35), 60 (29). HRMS (EI): calcd. for $\mathrm{C}_{25} \mathrm{H}_{16} \mathrm{O}_{4}$ $[\mathrm{M}]^{+\cdot}$ 380.1049; found 380.1048 .

3-(3,4-Dihydroxyphenyl)-2-(4-hydroxyphenyl)-9H-xanthen-9-one (13k): Reaction time 3 h, 94\% yield. This compound showed spectroscopic and analytical data identical to those previously reported. ${ }^{[27]}$

2,3-Bis(3,4-dihydroxyphenyl)-9H-xanthen-9-one (13I): Reaction time $4 \mathrm{~h}, 70 \%$ yield; m.p. $>340{ }^{\circ} \mathrm{C}$, dec. at $265^{\circ} \mathrm{C} .{ }^{1} \mathrm{H}$ NMR $\left(\left[D_{6}\right]-\right.$ DMSO): $\delta=6.42\left(\mathrm{dd}, J=8.1,2.1 \mathrm{~Hz}, 1 \mathrm{H}, 6^{\prime \prime}-\mathrm{H}\right), 6.53(\mathrm{dd}, J=$ 8.0, 2.2 Hz, $\left.1 \mathrm{H}, 6^{\prime}-\mathrm{H}\right), 6.61$ (d, $\left.J=2.1 \mathrm{~Hz}, 1 \mathrm{H}, 2^{\prime \prime}-\mathrm{H}\right), 6.65$ (d, $J$ $\left.=2.2 \mathrm{~Hz}, 1 \mathrm{H}, 2^{\prime}-\mathrm{H}\right), 6.66\left(\mathrm{~d}, J=8.0 \mathrm{~Hz}, 1 \mathrm{H}, 5^{\prime}-\mathrm{H}\right), 6.68(\mathrm{~d}, J=$ $\left.8.1 \mathrm{~Hz}, 1 \mathrm{H}, 5^{\prime \prime}-\mathrm{H}\right), 7.50$ (dt, $\left.J=7.7,0.8 \mathrm{~Hz}, 1 \mathrm{H}, 7-\mathrm{H}\right), 7.50$ (s, 1 H, 4-H), 7.69 (d, $J=8.0 \mathrm{~Hz}, 1 \mathrm{H}, 5-\mathrm{H}), 7.89$ (ddd, $J=8.0,7.7$, $1.7 \mathrm{~Hz}, 1 \mathrm{H}, 6-\mathrm{H}), 7.98$ (s, $1 \mathrm{H}, 1-\mathrm{H}), 8.22(\mathrm{dd}, J=7.7,1.7 \mathrm{~Hz}, 1$ 
$\mathrm{H}, 8-\mathrm{H}), 8.95,8.98$ and $9.14\left(3 \times \mathrm{s}, 4 \mathrm{H}, 3^{\prime}, 4^{\prime}, 3^{\prime \prime}, 4^{\prime \prime}-\mathrm{OH}\right) \mathrm{ppm} .{ }^{13} \mathrm{C}$ NMR ([D $]$ DMSO): $\delta=115.4$ and $115.5\left(\mathrm{C}-5^{\prime}, \mathrm{C}-5^{\prime \prime}\right), 116.8(\mathrm{C}-$ 2', C-2' '), 118.3 (C-5), 118.9 (C-4), 119.4 (C-9a), 120.6 (C-6"'), 120.7 (C-6'), 121.3 (C-8a), 124.4 (C-7), 126.1 (C-8), 127.0 (C-1), 130.7 (C-1' ), 131.1 (C-1'), 135.5 (C-6), 136.8 (C-3), 144.5 (C-4'), 144.9 (C-3', C-3' '), 145.3 (C-4'), 147.6 (C-2), 154.3 (C-4a), 155.8 (C-4b), 175.8 (C-9) ppm. EI-MS: $m / z(\%)=412[\mathrm{M}]^{+\cdot}(79), 396$ (17), 395 (16), 365 (15), 213 (26), 199 (17), 185 (30), 171 (25), 157 (20), 151 (18), 147 (17), 143 (22), 141 (17), 139 (19), 137 (22), 135 (20), 133 (20), 129 (43), 125 (31), 123 (31), 121 (30), 115 (23), 112 (32), 111 (60), 98 (96), 97 (89), 83 (100), 69 (30), 67 (33). HRMS (EI): calcd. for $\mathrm{C}_{25} \mathrm{H}_{16} \mathrm{O}_{6}[\mathrm{M}]^{+\cdot} 412.0947$; found 412.0955 .

6-Hydroxy-2,3-diphenyl-9H-xanthen-9-one (13m): Reaction time $72 \mathrm{~h}, 37 \%$ yield. ${ }^{1} \mathrm{H}$ NMR ([D $]$ DMSO): $\delta=6.91(\mathrm{~d}, J=2.1 \mathrm{~Hz}$, $1 \mathrm{H}, 5-\mathrm{H}), 6.94(\mathrm{dd}, J=8.7,2.1 \mathrm{~Hz}, 1 \mathrm{H}, 7-\mathrm{H}), 7.13-7.16(\mathrm{~m}, 2 \mathrm{H}$, $\left.2^{\prime}, 6^{\prime}-\mathrm{H}\right), 7.20-7.23$ (m, $\left.2 \mathrm{H}, 2^{\prime \prime}, 6^{\prime \prime}-\mathrm{H}\right), 7.26-7.31$ (m, $6 \mathrm{H}, 3^{\prime}, 4^{\prime}, 5^{\prime}-$ $\left.\mathrm{H}, 3^{\prime \prime}, 4^{\prime \prime}, 5^{\prime \prime}-\mathrm{H}\right), 7.63$ (s, $\left.1 \mathrm{H}, 4-\mathrm{H}\right), 8.07$ (s, $\left.1 \mathrm{H}, 1-\mathrm{H}\right), 8.08$ (d, $J$ $=8.7 \mathrm{~Hz}, 1 \mathrm{H}, 8-\mathrm{H}), 11.04(\mathrm{~s}, 1 \mathrm{H}, 6-\mathrm{OH}) \mathrm{ppm} .{ }^{13} \mathrm{C}$ NMR $\left(\left[\mathrm{D}_{6}\right]\right.$ DMSO): $\delta=102.8$ (C-5), 114.1 (C-7), 114.3 (C-8a), 119.4 (C-4), 120.2 (C-9a), 127.0 (C-4'), 127.4 (C-1), 127.7 (C-4'), 128.1 (C-8), $128.2\left(\mathrm{C}-3^{\prime}, 5^{\prime}, \mathrm{C}-3^{\prime \prime}, 5^{\prime \prime}\right), 129.4\left(\mathrm{C}-2^{\prime \prime}, 6^{\prime \prime}\right), 129.5\left(\mathrm{C}-2^{\prime}, 6^{\prime}\right), 136.4$ (C-2), 139.3 (C-1' ), 139.7 (C-1'), 146.5 (C-3), 154.7 (C-4a), 157.8 (C-4b), 164.1 (C-6), 174.5 (C-9) ppm.

1-Hydroxy-6,7-diphenyl-9H-xanthen-9-one (12n, 13n): Reaction time $1 \mathrm{~h}, 63 \%$ yield, see spectroscopic characterisation above. Also occurs as a by-product in the synthesis of 1-hydroxy-6,7-diphenyl9H-xanthen-9-one (5n).

1-Hydroxy-7-(4-hydroxyphenyl)-6-phenyl-9H-xanthen-9-one (13o): Reaction time $2 \mathrm{~h}, 90 \%$ yield; m.p. $130-133{ }^{\circ} \mathrm{C} .{ }^{1} \mathrm{H}$ NMR ([D 6 DMSO): $\delta=6.63$ (d, $\left.J=8.4 \mathrm{~Hz}, 2 \mathrm{H}, 3^{\prime}, 5^{\prime}-\mathrm{H}\right), 6.79$ (d, $J=8.4 \mathrm{~Hz}$, $1 \mathrm{H}, 7-\mathrm{H}), 6.87\left(\mathrm{~d}, J=8.4 \mathrm{~Hz}, 2 \mathrm{H}, 2^{\prime}, 6^{\prime}-\mathrm{H}\right), 7.00(\mathrm{~d}, J=8.3 \mathrm{~Hz}$, $1 \mathrm{H}, 5-\mathrm{H}), 7.15-7.18\left(\mathrm{~m}, 2 \mathrm{H}, 2^{\prime \prime}, 6^{\prime \prime}-\mathrm{H}\right), 7.29-7.31(\mathrm{~m}, 3 \mathrm{H}$, $\left.3^{\prime \prime}, 4^{\prime \prime}, 5^{\prime \prime}-\mathrm{H}\right), 7.49$ (s, $\left.1 \mathrm{H}, 4-\mathrm{H}\right), 7.70$ (dd, $J=8.4,8.3 \mathrm{~Hz}, 1 \mathrm{H}, 6-$ $\mathrm{H}), 7.91$ (s, $1 \mathrm{H}, 1-\mathrm{H}), 12.49$ (s, $1 \mathrm{H}, 8-\mathrm{OH}) \mathrm{ppm} .{ }^{13} \mathrm{C}$ NMR $\left(\left[\mathrm{D}_{6}\right]\right.$ DMSO): $\delta=107.6$ (C-5), 108.6 (C-8a), 110.6 (C-7), $115.4\left(\mathrm{C}-3^{\prime}, 5^{\prime}\right)$, 118.9 (C-9a), 119.5 (C-4), 126.5 (C-1), 128.1 (C-4' $), 128.5$ (C$\left.3^{\prime \prime}, 5^{\prime \prime}\right), 129.6\left(\mathrm{C}-2^{\prime \prime}, 6^{\prime \prime}\right), 130.1\left(\mathrm{C}-1^{\prime}\right), 130.9\left(\mathrm{C}-2^{\prime}, 6^{\prime}\right), 137.1(\mathrm{C}-2)$, 137.8 (C-6), 139.6 (C-1' '), 148.2 (C-3), 154.6 (C-4a), 156.1 (C-4b), $156.8\left(\mathrm{C}-4^{\prime}\right), 161.2(\mathrm{C}-8), 181.6(\mathrm{C}-9)$ ppm. EI-MS: $m / z(\%)=380$ $[\mathrm{M}]^{+\cdot}(100), 379$ (20), 351 (6). HRMS (EI): calcd. for $\mathrm{C}_{25} \mathrm{H}_{16} \mathrm{O}_{4}$ $[\mathrm{M}]^{+\cdot} 380.1049$; found 380.1046 .

7-(3,4-Dihydroxyphenyl)-1-hydroxy-6-phenyl-9H-xanthen-9-one (13p): Reaction time $3 \mathrm{~h}, 83 \%$ yield; m.p. $222-224{ }^{\circ} \mathrm{C} .{ }^{1} \mathrm{H}$ NMR ([D $]$ DMSO): $\delta=6.32\left(\mathrm{dd}, J=8.1,2.1 \mathrm{~Hz}, 1 \mathrm{H}, 6^{\prime}-\mathrm{H}\right), 6.54(\mathrm{~d}, J$ $\left.=2.1 \mathrm{~Hz}, 1 \mathrm{H}, 2^{\prime}-\mathrm{H}\right), 6.60\left(\mathrm{~d}, J=8.1 \mathrm{~Hz}, 1 \mathrm{H}, 5^{\prime}-\mathrm{H}\right), 6.79(\mathrm{~d}, J=$ $8.3 \mathrm{~Hz}, 1 \mathrm{H}, 7-\mathrm{H}), 7.01(\mathrm{~d}, J=8.3 \mathrm{~Hz}, 1 \mathrm{H}, 5-\mathrm{H}), 7.17-7.20(\mathrm{~m}, 2$ $\left.\mathrm{H}, 2^{\prime \prime}, 6^{\prime \prime}-\mathrm{H}\right), 7.30-7.32$ (m, $\left.3 \mathrm{H}, 3^{\prime \prime}, 4^{\prime \prime}, 5^{\prime \prime}-\mathrm{H}\right), 7.48$ (s, $\left.1 \mathrm{H}, 4-\mathrm{H}\right)$, 7.70 (t, $J=8.3 \mathrm{~Hz}, 1 \mathrm{H}, 6-\mathrm{H}), 7.91$ (s, $1 \mathrm{H}, 1-\mathrm{H}), 12.48$ (s, $1 \mathrm{H}, 8-$ $\mathrm{O} H$ ) ppm. ${ }^{13} \mathrm{C}$ NMR ([D $]$ DMSO): $\delta=107.8$ (C-5), 108.7 (C-8a), 110.7 (C-7), 115.8 (C-5'), 117.3 (C-2'), 118.9 (C-9a), 119.6 (C-4), $121.2\left(\mathrm{C}-6^{\prime}\right), 126.6(\mathrm{C}-1), 128.3\left(\mathrm{C}-4^{\prime \prime}\right), 128.7\left(\mathrm{C}-3^{\prime \prime}{ }^{\prime} 5^{\prime \prime}\right), 129.6(\mathrm{C}-$ $\left.2^{\prime \prime}, 6^{\prime \prime}\right), 130.9\left(\mathrm{C}-1^{\prime}\right), 137.4(\mathrm{C}-2), 138.0(\mathrm{C}-6), 139.7\left(\mathrm{C}-1^{\prime \prime}\right), 145.0$ (C-4'), 145.3 (C-3'), 148.3 (C-3), 154.7 (C-4a), 156.2 (C-4b), 161.2 (C-8), 181.7 (C-9) ppm. EI-MS: $m / z(\%)=396[\mathrm{M}]^{+\cdot}(100), 380$ (15), 379 (15), 349 (16), 213 (27), 199 (18), 185 (19), 171 (24), 157 (20), 147 (40), 143 (25), 137 (28), 129 (40), 125 (32), 123 (29), 121 (34), 111 (48), 98 (77), 97 (98), 96 (45), 95 (53), 85 (36), 83 (77), 81 (47), 73 (40), 67 (29). HRMS (EI): calcd. for $\mathrm{C}_{25} \mathrm{H}_{16} \mathrm{O}_{5}[\mathrm{M}]^{+\cdot}$ 396.0998; found 396.0996 .

6-(3,4-Dihydroxyphenyl)-1-hydroxy-7-phenyl-9H-xanthen-9-one (13q): Reaction time 3 h, $71 \%$ yield; m.p. 205-207 ${ }^{\circ} \mathrm{C} .{ }^{1} \mathrm{H}$ NMR ([D $]$ DMSO): $\delta=6.47$ (dd, $\left.J=8.2,2.2 \mathrm{~Hz}, 1 \mathrm{H}, 6^{\prime \prime}-\mathrm{H}\right), 6.61(\mathrm{~d}, J$ $\left.=2.2 \mathrm{~Hz}, 1 \mathrm{H}, 2^{\prime \prime}-\mathrm{H}\right), 6.65\left(\mathrm{~d}, J=8.2 \mathrm{~Hz}, 1 \mathrm{H}, 5^{\prime \prime}-\mathrm{H}\right), 6.84$ (dd, $J$ $=8.2,0.6 \mathrm{~Hz}, 1 \mathrm{H}, 7-\mathrm{H}), 7.09(\mathrm{~d}, J=8.2 \mathrm{~Hz}, 1 \mathrm{H}, 5-\mathrm{H}), 7.17-7.20$ (m, $\left.2 \mathrm{H}, 2^{\prime}, 6^{\prime}-\mathrm{H}\right), 7.28-7.36\left(\mathrm{~m}, 3 \mathrm{H}, 3^{\prime}, 4^{\prime}, 5^{\prime}-\mathrm{H}\right), 7.54$ (s, $1 \mathrm{H}, 4-$ $\mathrm{H}), 7.75$ (t, $J=8.2 \mathrm{~Hz}, 1 \mathrm{H}, 6-\mathrm{H}), 8.01(\mathrm{~s}, 1 \mathrm{H}, 1-\mathrm{H}), 8.99$ and 9.20 $\left(2 \times\right.$ brs, $\left.2 \mathrm{H}, 3^{\prime \prime}, 4^{\prime \prime}-\mathrm{OH}\right), 12.59$ (s, $\left.1 \mathrm{H}, 8-\mathrm{OH}\right) \mathrm{ppm} .{ }^{13} \mathrm{C} \mathrm{NMR}$ ([D 6 DMSO): $\delta=107.4$ (C-5), 108.4 (C-8a), 110.4 (C-7), 115.5 (C5'), 116.9 (C-2' ), 118.3 (C-9a), 118.8 (C-4), 120.9 (C-6"'), 126.7 (C-1), $127.1\left(\mathrm{C}-4^{\prime}\right), 128.3\left(\mathrm{C}-3^{\prime}, 5^{\prime}\right), 129.3\left(\mathrm{C}-2^{\prime}, 6^{\prime}\right), 130.1\left(\mathrm{C}-1^{\prime \prime}\right)$, 136.7 (C-2), 137.5 (C-6), 139.8 (C-1'), 145.0 (C-3"'), 145.5 (C-4" $)$, 148.6 (C-3), 154.8 (C-4a), 155.9 (C-4b), 161.0 (C-8), 181.4 (C9) ppm. EI-MS: $m / z(\%)=396[\mathrm{M}]^{+\cdot}(100), 395$ (7), 380 (9), 379 (9), 349 (7). HRMS (EI): calcd. for $\mathrm{C}_{25} \mathrm{H}_{16} \mathrm{O}_{5}[\mathrm{M}]^{+\cdot} 396.0998$; found 396.1003 .

1-Hydroxy-6,7-bis(3,4-dihydroxyphenyl)-9H-xanthen-9-one (13r): Reaction time 5 h, $90 \%$ yield; m.p. $>340{ }^{\circ} \mathrm{C}$, dec. at $305^{\circ} \mathrm{C} .{ }^{1} \mathrm{H}$ NMR ([D $\left.\left.]_{6}\right] \mathrm{DMSO}\right): \delta=6.42\left(\mathrm{dd}, J=8.0,2.1 \mathrm{~Hz}, 1 \mathrm{H}, 6^{\prime}-\mathrm{H}\right), 6.52$ $\left(\mathrm{dd}, J=8.2,2.2 \mathrm{~Hz}, 1 \mathrm{H}, 6^{\prime \prime}-\mathrm{H}\right), 6.60\left(\mathrm{~d}, J=2.1 \mathrm{~Hz}, 1 \mathrm{H}, 2^{\prime}-\mathrm{H}\right)$, $6.65\left(\mathrm{~d}, J=2.2 \mathrm{~Hz}, 1 \mathrm{H}, 2^{\prime \prime}-\mathrm{H}\right), 6.66\left(\mathrm{~d}, J=8.0 \mathrm{~Hz}, 1 \mathrm{H}, 5^{\prime}-\mathrm{H}\right)$, $6.68\left(\mathrm{~d}, J=8.2 \mathrm{~Hz}, 1 \mathrm{H}, 5^{\prime \prime}-\mathrm{H}\right), 6.84(\mathrm{dd}, J=8.4,0.7 \mathrm{~Hz}, 1 \mathrm{H}, 7-$ H), 7.09 (dd, $J=8.4,0.7 \mathrm{~Hz}, 1 \mathrm{H}, 5-\mathrm{H}), 7.49(\mathrm{~s}, 1 \mathrm{H}, 4-\mathrm{H}), 7.74(\mathrm{t}$, $J=8.4 \mathrm{~Hz}, 1 \mathrm{H}, 6-\mathrm{H}), 7.95(\mathrm{~s}, 1 \mathrm{H}, 1-\mathrm{H}), 8.95$ (s, $\left.1 \mathrm{H}, 4^{\prime}-\mathrm{OH}\right), 8.98$ (s, $\left.1 \mathrm{H}, 4^{\prime \prime}-\mathrm{OH}\right), 9.00$ and $9.17\left(2 \times \mathrm{s}, 2 \mathrm{H}, 3^{\prime}, 3^{\prime \prime}-\mathrm{OH}\right), 12.63$ (s, 1 $\mathrm{H}, 8-\mathrm{OH}$ ) ppm. ${ }^{13} \mathrm{C}$ NMR ([D 6 DMSO): $\delta=107.4$ (C-5), $108.4(\mathrm{C}-$ $8 \mathrm{a}), 110.3$ (C-7), 115.46 and 115.53 (C-5', C-5' $), 116.8$ (C-2', C2"), 118.2 (C-9a), 118.7 (C-4), 120.5 (C-6'), 120.7 (C-6"), 126.3 (C-1), 130.5 (C-1'), 130.9 (C-1'), 137.0 (C-2), 137.4 (C-6), 144.6 (C-4'), 144.9 and 145.0 (C-3', C-3' $), 145.4\left(\mathrm{C}-4^{\prime \prime}\right), 148.5$ (C-3), 154.4 (C-4a), 156.0 (C-4b), 161.0 (C-8), 181.5 (C-9) ppm. EI-MS: $m / z(\%)=428[\mathrm{M}]^{+\cdot}(100), 396(24), 381(18), 213(24), 191$ (21), 185 (30), 129 (43), 125 (25), 123 (27), 121 (27), 115 (20), 112 (32), 111 (46), 107 (25), 105 (24), 98 (75), 97 (92), 95 (43), 85 (33), 83 (83), 81 (37), 73 (34), 71 (36), 67 (30). HRMS (EI): calcd. for $\mathrm{C}_{25} \mathrm{H}_{16} \mathrm{O}_{7}[\mathrm{M}]^{+\cdot}$ 428.0896; found 428.0888 .

1,3-Dihydroxy-6,7-diphenyl-9H-xanthen-9-one (13s): Reaction time $120 \mathrm{~h}, 60 \%$ yield. ${ }^{1} \mathrm{H}$ NMR ([D $]$ DMSO): $\delta=6.24(\mathrm{~d}, J=1.9 \mathrm{~Hz}$, $1 \mathrm{H}, 7-\mathrm{H}), 6.43(\mathrm{~d}, J=1.9 \mathrm{~Hz}, 1 \mathrm{H}, 5-\mathrm{H}), 7.13-7.31(\mathrm{~m}, 10 \mathrm{H}, 2,3-$ $\left.\mathrm{C}_{6} H_{5}\right), 7.61$ (s, $\left.1 \mathrm{H}, 4-\mathrm{H}\right), 8.02$ (s, $\left.1 \mathrm{H}, 1-\mathrm{H}\right), 11.23$ (brs, $1 \mathrm{H}, 6-$ $\mathrm{O} H$ ), 12.80 (s, $1 \mathrm{H}, 8-\mathrm{O} H$ ) ppm. ${ }^{13} \mathrm{C}$ NMR ([D 6$]$ DMSO): $\delta=94.3$ (C-5), 98.3 (C-7), 102.4 (C-8a), 118.9 (C-4), 119.2 (C-9a), 126.6 (C1), $127.2\left(\mathrm{C}-4^{\prime}\right), 127.9\left(\mathrm{C}-4^{\prime \prime}\right), 128.3\left(\mathrm{C}-3^{\prime}, 5^{\prime}, \mathrm{C}-3^{\prime \prime}, 5^{\prime \prime}\right), 129.5(\mathrm{C}-$ $\left.2^{\prime \prime}, 6^{\prime \prime}\right), 129.6\left(\mathrm{C}-2^{\prime}, 6^{\prime}\right), 136.6(\mathrm{C}-2), 139.2\left(\mathrm{C}-1^{\prime \prime}\right), 139.5\left(\mathrm{C}-1^{\prime}\right)$, 147.3 (C-3), 154.5 (C-4a), 157.6 (C-4b), 162.9 (C-8), 166.0 (C-6), 179.4 (C-9) ppm. EI-MS: $m / z(\%)=380[\mathrm{M}]^{+\cdot}(100), 379(35), 365$ (7), 228 (5), 84 (10), 66 (11). HRMS (EI): calcd. for $\mathrm{C}_{25} \mathrm{H}_{16} \mathrm{O}_{4}$ $[\mathrm{M}]^{+\cdot}$ 380.1049; found 380.1045 .

\section{Acknowledgments}

Sincere thanks are expressed to the University of Aveiro, Fundação para a Ciência e a Tecnologia (FCT), and Fundo Europeu de Desenvolvimento Regional (FEDER) for funding the Organic Chemistry Research Unit and the project POCI/QUI/59284/2004. One of us (C. M. M. S.) is also grateful to Programa de Desenvolvimento Educativo para Portugal (PRODEP 5.3) for financial support.

[1] J. C. Roberts, Chem. Rev. 1961, 38, 591-605.

[2] L. M. M. Vieira, A. Kijjoa, Curr. Med. Chem. 2005, 12, 2413 2446.

[3] G. J. Bennett, H.-H. Lee, Phytochemistry 1989, 28, 967-998.

[4] S. Sakai, M. Katsura, H. Takayama, N. Aimi, N. Chokethaworn, M. Suttajit, Chem. Pharm. Bull. 1993, 41, 958-960.

[5] I. Fukawa, H. Yoneda, K. K. K. K. Asahi, European Patent EP0237004, Sep, 1987. 
[6] A. S. Kelkar, R. M. Letcher, K.-K. Cheung, K.-F. Chiu, G. D. Brown, J. Chem. Soc. Perkin Trans. 1 2000, 3732-3741.

[7] A. Abdel-Lateff, C. Klemke, G. M. König, A. D. Wright, J. Nat. Prod. 2003, 66, 706-708.

[8] T. Fukai, M. Yonekawa, A.-J. Hou, T. Nomura, H.-D. Sun, J. Uno, J. Nat. Prod. 2003, 66, 1118-1120.

[9] M. M. Mackeen, A. M. Ali, N. H. Lajis, K. Kawazu, Z. Hassan, M. Amran, M. Habsah, L. Y. Mooi, S. M. Mohamed, J. Ethnopharmacol. 2000, 72, 395-402.

[10] H. H. Park, Y.-D. Park, J.-M. Han, K.-R. Im, B. W. Lee, I. Y. Jeong, T.-S. Jeong, W. S. Lee, Bioorg. Med. Chem. Lett. 2006, 16, 5580-5583.

[11] C. N. Lin, M. I. Chung, S. J. Liou, T. H. Lee, J. P. Wang, J. Pharm. Pharmacol. 1996, 48, 532-538.

[12] M. Riscoe, J. X. Kelly, R. Winter, Curr. Med. Chem. 2005, 12, 2539-2549.

[13] C. Portela, C. M. M. Afonso, M. M. M. Pinto, M. J. Ramos, Bioorg. Med. Chem. 2004, 12, 3313-3321.

[14] V. K. Dua, V. P. Ojha, R. Roy, B. C. Joshi, N. Valecha, C. U. Devi, M. C. Batnagar, V. P. Sharma, S. K. Subbarao, J. Ethnopharmacol. 2004, 95, 247-251.

[15] H.-Z. Zhang, S. Kasibhatla, Y. Wang, J. Herich, J. Guastella, B. Tseng, J. Drewe, S. X. Cai, Bioorg. Med. Chem. 2004, 12, 309-317.

[16] G. W. Rewcastle, G. J. Atwell, L. Zhang, B. C. Baguley, W. A. Denny, J. Med. Chem. 1991, 34, 217-222.

[17] J. Merza, M.-C. Aumond, D. Rondeau, V. Dumontet, A.-M. Le Ray, D. Séraphin, P. Richomme, Phytochemistry 2004, 65, 2915-2920.
[18] A.-E. Hay, M.-C. Aumond, S. Mallet, V. Dumontet, M. Litaudon, D. Rondeau, P. Richomme, J. Nat. Prod. 2004, 67, 707709 .

[19] P. M. Pauletti, I. Castro-Gamboa, D. H. S. Silva, M. C. M. Young, D. M. Tomazela, M. N. Eberlin, V. S. Bolzani, J. Nat. Prod. 2003, 66, 1384-1387.

[20] M. M. M. Pinto, M. E. Sousa, M. S. J. Nascimento, Curr. Med. Chem. 2005, 12, 2517-2538.

[21] W. Bors, W. Heller, C. Michel, K. Stettmaier, in: Handbook of Antioxidants (Ed.: E. Cadenas, L. Packer), Marcel Dekker, Inc., New York, 1996, pp. 409-466.

[22] M. E. Sousa, M. M. M. Pinto, Curr. Med. Chem. 2005, 12, 2447-2479.

[23] L. K. Cassilas, C. A. Townsend, J. Org. Chem. 1999, 64, 40504059.

[24] M. Afzal, J. M. Al-Hassan, Heterocycles 1980, 14, 1173-1205.

[25] S. S. Ibrahim, Ind. Eng. Chem. Res. 2001, 40, 37-39.

[26] C. M. M. Santos, A. M. S. Silva, J. A. S. Cavaleiro, Synlett 2005, 3095-3098.

[27] C. M. M. Santos, A. M. S. Silva, J. A. S. Cavaleiro, Synlett 2007, 3113-3116.

[28] A. M. S. Silva, D. C. G. A. Pinto, J. A. S. Cavaleiro, A. Lévai, T. Patonay, Arkivoc 2004, 6, 106-123.

[29] A. Fougerousse, E. Gonzalez, R. Brouillard, J. Org. Chem. 2000, 65, 583-586.

[30] S. Punna, U. H. Meunier, M. G. Finn, Org. Lett. 2004, 6, $2777-$ 2779.

[31] T. Nordvik, U. H. Brinker, J. Org. Chem. 2003, 68, 9394-9399.

[32] D. C. G. A. Pinto, A. M. S. Silva, L. M. P. M. Almeida, J. A. S. Cavaleiro, A. Lévai, T. Patonay, J. Heterocycl. Chem. 1998, 35, 217-224. 